\title{
The Eigenvalues of Hyperoctahedral Descent Operators and Applications to Card-Shuffling
}

\author{
C.Y. Amy Pang* \\ Department of Mathematics \\ Hong Kong Baptist University \\ Hong Kong, China. \\ amypang@hkbu.edu.hk
}

Submitted: Aug 28, 2021; Accepted: Feb 4, 2022; Published: Feb 25, 2022

(C) The author. Released under the CC BY-ND license (International 4.0).

\begin{abstract}
We extend an algebra of Mantaci and Reutenauer, acting on the free associative algebra, to a vector space of operators acting on all graded connected Hopf algebras. These operators are convolution products of certain involutions, which we view as hyperoctahedral variants of Patras's descent operators. We obtain the eigenvalues and multiplicities of all our new operators, as well as a basis of eigenvectors for a subclass akin to Adams operations. We outline how to apply this eigendata to study Markov chains, and examine in detail the case of card-shuffles with flips or rotations.
\end{abstract}

Mathematics Subject Classifications: 05C88, 05C89

\section{Introduction}

The use of graded Hopf algebras to study combinatorics is increasingly common [JR79, Hiv07, ABS06, AA17]. These are graded vector spaces $\mathcal{H}=\bigoplus_{n=0}^{\infty} \mathcal{H}_{n}$ with bases indexed by combinatorial objects, such as trees [LR98, CP17], graphs [Sch93] or permutations [MR95a], admitting a product $m: \mathcal{H} \otimes \mathcal{H} \rightarrow \mathcal{H}$ and coproduct $\Delta: \mathcal{H} \rightarrow \mathcal{H} \otimes \mathcal{H}$ that encode respectively how these objects combine and break apart. The composition $m \circ \Delta$, or more generally the composition of iterated product and coproduct maps $m^{[a]} \circ \Delta^{[a]}: \mathcal{H} \rightarrow$ $\mathcal{H}^{\otimes a} \rightarrow \mathcal{H}$, are the Adams operations, studied in [Lod98, Sec. 4.5] for their connections to Hochschild homology. Their eigenvalues and multiplicities are obtained in [AL15], and applied to derive some combinatorial identities. The paper [DPR14] gives a basis of

\footnotetext{
*Supported by RGC-ECS grant 22300017.
} 
eigenvectors for $m^{[a]} \circ \Delta^{[a]}$ on free-commutative or cocommutative algebras, and interprets the matrix of these Adams operations as the transition probabilities of a Markov chain on a basis of $\mathcal{H}$. When $\mathcal{H}$ is the shuffle algebra, this probabilistic interpretation recovers the famous Gilbert-Shannon-Reeds riffle-shuffle of a deck of cards [BD92]: cut the deck into $a$ piles according to the multinomial distribution, then interleave the piles together so cards from the same pile stay in the same relative order. The eigenvectors (on the dual algebra) then lead to bounds for certain probabilities under repeated shuffles.

We may doctor the Adams operations by projecting to a particular graded subspace after taking the coproduct and before the product; this results in maps of the form $m^{[a]} \circ \Delta_{\left(d_{1}, \ldots, d_{a}\right)}: \mathcal{H} \rightarrow \mathcal{H}_{d_{1}} \otimes \cdots \otimes \mathcal{H}_{d_{a}} \rightarrow \mathcal{H}$, the descent operators of Patras [Pat94], closely related to Solomon's descent algebra of the symmetric group [Sol76]. The paper [Pan18] finds the eigenvalues and eigenvectors of their linear combinations, which correspond to more general card shuffles where the deck is cut into $a$ piles according to other distributions, related to the Tsetlin library models of dynamic storage allocation [Fil96]. Although much is already known about such shuffles, the Hopf-algebraic viewpoint means that the same eigendata formula can be applied to analyse a diverse range of analogous "shuffling" Markov chains on various combinatorial objects; for example, setting $\mathcal{H}$ to be the Loday-Ronco Hopf algebra on binary trees generates a chain on trees that removes some vertices and reattaches them elsewhere.

The present paper presents analogous results on the new class of hyperoctahedral descent operators, motivated by numerous card-shuffling models where, after cutting the deck, some piles are flipped over before combining [BB92, DFH13, PP98, BW17]; see Corollary 36 for the precise definition. Our hyperoctahedral descent operators are linear combinations of maps of the form $m \circ\left(f_{1} \otimes \cdots \otimes f_{a}\right) \circ \Delta_{\left(d_{1}, \ldots, d_{a}\right)}$, where each $f_{i}$ is the identity or an involution $\tau$ (satisfying certain conditions). These generalise the MantaciReutenauer algebra of endomorphisms on the free associative algebra [MR95b]. Our main algebraic results, in Sections 2 and 3, are:

- Proposition 12, that the composition of hyperoctahedral descent operators on a commutative or cocommutative Hopf algebra follows the multiplication in the MantaciReutenauer algebra;

- Theorem 17, a uniform expression for the eigenvalues and multiplicities for any hyperoctahedral descent operator;

- Theorems 26, 27 and 29, a complete basis for the non-zero eigenspaces of

$$
m^{[a]} \circ(\mathrm{id} \otimes \tau \otimes \mathrm{id} \otimes \tau \otimes \ldots) \circ \Delta^{[a]}
$$

on cocommutative Hopf algebras. This is one analogue of an Adams operation, corresponding to the hyperoctahedral riffle-shuffles of [BD92, BB92] where, after cutting the deck, every other pile is flipped upside-down before the interleaving step.

The remainder of the paper concerns probabilistic connections. We show in Theorem 34 that, under mild positivity conditions, the matrix for a hyperoctahedral descent operator on an arbitrary graded Hopf algebra $\mathcal{H}$ can be rescaled into a matrix of transition 
probabilities, using the same formula as for usual ("type A") descent operators. As in the type A case, the stationary distributions of these chains are independent of which hyperoctahedral descent operator is used; an explicit description of these distributions are in Theorem 35. We then take $\mathcal{H}$ to be a signed shuffle algebra, to analyse two card shuffles related to (1) (by using different $\tau$ ): the riffle-shuffle that flips every other pile, as mentioned above, and a relatively unstudied riffle-shuffle that rotates every other pile by 180 degrees. (The only prior work on the latter known to the author is the eigenvalue calculation in the unpublished manuscript [DS] using coloured versions of left regular bands.) Specialisation of the general formulas gives a complete basis for the non-zero eigenspaces in the language of Lyndon words. From these eigenvectors we deduce (starting at the identity permutation of cards):

- Proposition 53: after $t$ iterations of a riffle-shuffle with flip on a deck of $n$ cards, the expected number of an unconventional type of descent (see Definition 50) is $\left(1-a^{-t}\right) \frac{n-1}{2}+a^{-t}$.

- Proposition 55: after $t$ iterations of a riffle-shuffle with flip (resp. rotation) on a deck of $n$ cards with $n$ even, and with an odd number of piles $a$, the probability of having sign +1 is $\frac{1}{2}\left(1+a^{-n t}\right)$ (resp. $\left.\frac{1}{2}\left(1+a^{-n t / 2}\right)\right)$. The flip case is a new proof of [FKLP21, Th. 5.8].

Thus this paper joins a long list of works calculating eigendata for shuffling models [PP99, BHR99, Wil04, DS18, Laf20]. As in the type A case, our eigenvector formulas apply to all graded connected Hopf algebras, and an interesting direction for future work is to obtain similar expectation results for "flipped shuffles" of trees or permutations. We note that Markov chains on signed permutations appear in the study of genome rearrangement [LSK02, Dur08], to compare the chromosomes of two species. Further, since the theory of the Mantaci-Reutenauer algebra extends easily to cyclic operators in place of involutions [MR95b, Sec. 6], the results here should be generalisable to the much greater class of "cyclic descent operators" without much complications, diagonalising the coloured shuffles of [Moy15].

The paper is structured as follows: in the algebraic part, Section 2 defines the hyperoctahedral descent operators and derives their composition law and eigenvalues; Section 3 specialises to $m^{[a]} \circ(\iota \otimes \tau \otimes \iota \otimes \tau \otimes \ldots) \circ \Delta^{[a]}$, finding their eigenvectors. In the probabilistic part, Section 4 associates a Markov chain to each hyperoctahedral descent operator acting on a graded connected Hopf algebra and give their general properties; Section 5 focuses on riffle-shuffles with flips or rotations..

Following the related Coxeter nomenclature, we refer to our new hyperoctahedral theory as "type B", and the original descent operators as "type A".

\section{Hyperoctahedral Descent Operators}

This work concerns graded connected Hopf algebras - that is, a graded vector space $\mathcal{H}=$ $\bigoplus_{n=0}^{\infty} \mathcal{H}_{n}$ (over $\mathbb{R}$ ) equipped with a linear product map $m: \mathcal{H}_{i} \otimes \mathcal{H}_{j} \rightarrow \mathcal{H}_{i+j}$ and a linear 
coproduct map $\Delta: \mathcal{H}_{n} \rightarrow \bigoplus_{i=0}^{n} \mathcal{H}_{i} \otimes \mathcal{H}_{n-i}$ satisfying certain associativity, coassociativity, and compatibility axioms [GR14]. Connectedness means that $\operatorname{dim} \mathcal{H}_{0}=1$, so we will identify $\mathcal{H}_{0}$ with $\mathbb{R}$, writing $\mathcal{H}_{0}=\operatorname{span}\{1\}$. In many examples where $\mathcal{H}$ has a basis indexed by combinatorial objects, $m$ and $\Delta$ can be interpreted respectively as rules for combining and breaking these objects, as in our two main examples:

Example 1. Fix $N \in \mathbb{N}$, and consider $\mathcal{A}:=\{1,2, \ldots, N, \overline{1}, \overline{2}, \ldots, \bar{N}\}$, where $\bar{i}$ represents a "negative" version of $i$. We may consider the shuffle algebra on this alphabet, whose basis is the set of words $w_{1} \ldots w_{n}$ on $\mathcal{A}$, representing a deck of cards with card $w_{1}$ on top, card $w_{2}$ second from the top, and so on, so card $w_{n}$ is at the bottom. There are two useful interpretations for $\bar{i}$ : either it is a rotation of card $i$ by $180^{\circ}$, or it is card $i$ flipped upside down. We may call this the signed shuffle algebra to differentiate it from the (unsigned) shuffle algebra on the alphabet $\{1,2, \ldots, N\}$, which is a quotient under the identification of $i$ with $\bar{i}$.

The degree of a word is its number of letters, i.e. the number of cards in the deck. The product of two words $u$ and $v$ is the sum of all their interleavings (meaning all words containing the letters in $u$ and $v$, in the same relative order as in $u$ and $v$ ), representing the shuffling of two decks. The coproduct is deconcatenation, or cutting the deck:

$$
\Delta\left(w_{1} \ldots w_{n}\right)=\sum_{i=0}^{n} w_{1} \ldots w_{i} \otimes w_{i+1} \ldots w_{n} .
$$

For example:

$$
\begin{aligned}
m(1 \overline{5} \otimes \overline{3} \overline{2})=(1 \overline{5})(\overline{3} \overline{2}) & =1 \overline{5} \overline{3} \overline{2}+1 \overline{3} \overline{5} \overline{2}+1 \overline{3} \overline{2} \overline{5}+\overline{3} 1 \overline{5} \overline{2}+\overline{3} 1 \overline{2} \overline{5}+\overline{3} \overline{2} 1 \overline{5} \\
\Delta(3 \overline{1} 6) & =\varnothing \otimes 3 \overline{1} 6+3 \otimes \overline{1} 6+3 \overline{1} \otimes 6+3 \overline{1} 6 \otimes \varnothing .
\end{aligned}
$$

As Section 5.1 will explain, the action of hyperoctahedral descent operators on the signed shuffle algebra induce card-shuffling models.

Example 2. On the alphabet $\mathcal{A}$ of the previous example, we may construct a free associative algebra, which for clarity we'll term the signed free associative algebra. Its basis is also the set of words on $\mathcal{A}$. The product is concatenation, representing placing one deck above another, and the coproduct is "deshuffling", i.e.

$$
\begin{aligned}
m\left(u_{1} \ldots u_{n} \otimes v_{1} \ldots v_{m}\right) & =u_{1} \ldots u_{n} v_{1} \ldots v_{m} \\
\Delta\left(w_{1} \ldots w_{n}\right) & =\sum_{S \subseteq\{1,2, \ldots, N\}} \prod_{i \in S} w_{i} \otimes \prod_{i \notin S} w_{i} .
\end{aligned}
$$

For example:

$$
\begin{aligned}
m(1 \overline{5} \otimes \overline{3} \overline{2})=(1 \overline{5})(\overline{3} \overline{2})= & 1 \overline{5} \overline{3} \overline{2} \\
\Delta(3 \overline{1} 6)= & \varnothing \otimes 3 \overline{1} 6+3 \otimes \overline{1} 6+\overline{1} \otimes 36+6 \otimes 3 \overline{1} \\
& +3 \overline{1} \otimes 6+36 \otimes \overline{1}+\overline{1} 6 \otimes 3+3 \overline{1} 6 \otimes \varnothing .
\end{aligned}
$$


As explained five paragraphs below, the signed free associative algebra is dual to the signed shuffle algebra, which means the eigenvectors for hyperocatedral descent operators acting on the signed free associative algebra give probabilistic results about card shuffles - see Section 5.5.

Note that the product structures of the above two examples were considered in [BB92].

In any Hopf algebra, we abuse notation and write $m$ for the product of more than two factors, e.g. in the signed shuffle algebra,

$$
\begin{gathered}
m(1 \overline{5} \otimes \overline{3} \otimes \overline{2})=1 \overline{5} \overline{3} \overline{2}+1 \overline{5} \overline{2} \overline{3}+1 \overline{2} \overline{5} \overline{3}+\overline{2} 1 \overline{5} \overline{3}+1 \overline{3} \overline{5} \overline{2}+1 \overline{3} \overline{2} \overline{5}+1 \overline{2} \overline{3} \overline{5}+\overline{2} 1 \overline{3} \overline{5} \\
+\overline{3} 1 \overline{5} \overline{2}+\overline{3} 1 \overline{2} \overline{5}+\overline{3} \overline{2} 1 \overline{5}+\overline{2} 31 \overline{5} .
\end{gathered}
$$

Dually, because of coassociativity, there is a well-defined notion of splitting a combinatorial object into $l$ parts: $\Delta^{[l]}: \mathcal{H} \rightarrow \mathcal{H}^{\otimes l}$ defined inductively by $\Delta^{[2]}=\Delta, \Delta^{[l]}=\left(\Delta^{[l-1]} \otimes\right.$ id $) \circ \Delta$. It is useful to constrain the "sizes of the parts" by projecting to graded subspaces after applying $\Delta^{[l]}$. Namely, for a weak-composition $D=\left(d_{1}, \ldots, d_{l(D)}\right)$ of $n$ (i.e. non-negative integers $d_{i}$ summing to $n$ ), set $\Delta_{D}: \mathcal{H}_{n} \rightarrow \mathcal{H}_{d_{1}} \otimes \cdots \otimes \mathcal{H}_{d_{l(D)}}$. For example, in the signed shuffle algebra, $\Delta_{2,1,1}(1 \overline{5} \overline{3} \overline{2})=1 \overline{5} \otimes \overline{3} \otimes \overline{2}$ and $\Delta^{[3]}(1 \overline{5} \overline{3} \overline{2})=1 \overline{5} \otimes \overline{3} \otimes \overline{2}+1 \otimes \overline{5} \overline{3} \otimes \overline{2}+1 \overline{5} \overline{3} \otimes$ $\varnothing \otimes \overline{2}+\varnothing \otimes \varnothing \otimes 1 \overline{5} \overline{3} \overline{2}+\ldots$ (11 more terms $)$.

A key idea for determining the spectrum and eigenvectors of hyperoctahedral descent operators is the primitive subspace of a Hopf algebra: $\operatorname{Prim}(\mathcal{H})=\{x \in \mathcal{H} \mid \Delta(x)=$ $1 \otimes x+x \otimes 1\}$. Because of the counit axiom, $(\operatorname{Prim} \mathcal{H}) \cap \mathcal{H}_{n}$ is equivalently characterised by $\left\{x \in \mathcal{H}_{n} \mid \Delta(x) \subseteq \mathcal{H}_{0} \otimes \mathcal{H}_{n}+\mathcal{H}_{n} \otimes \mathcal{H}_{0}\right\}$. Prim $(\mathcal{H})$ is a Lie algebra, meaning that it is closed under the Lie bracket: if $x, y \in \operatorname{Prim}(\mathcal{H})$, then $[x, y]:=x y-y x \in \operatorname{Prim}(\mathcal{H})$.

A Hopf algebra $\mathcal{H}$ is commutative if $w z=z w$ for all $w, z \in \mathcal{H}$. And $\mathcal{H}$ is cocommutative if, for all $x \in \mathcal{H}$, its coproduct $\Delta(x)$ is invariant under the swapping of tensor-factors, i.e. $\Delta(x)=\sum_{i} w_{i} \otimes z_{i}=\sum_{i} z_{i} \otimes w_{i}$.

Given a graded connected Hopf algebra $\mathcal{H}=\bigoplus_{n \geqslant 0} \mathcal{H}_{n}$, the symmetry of the Hopf axioms allows the definition of a Hopf structure on the (graded) dual vector space $\mathcal{H}^{*}:=$ $\oplus_{n \geqslant 0} \mathcal{H}_{n}^{*}$ : for $f, g \in \mathcal{H}^{*}$, set

$$
m(f \otimes g)(x):=(f \otimes g)(\Delta x), \quad \Delta(f)(w \otimes z)=f(w z),
$$

with $x, z, w \in \mathcal{H}$. (Here, $(f \otimes g)(a \otimes b)=f(a) g(b)$.) Note that the dual of a commutative Hopf algebra is cocommutative, and vice versa.

The signed shuffle algebra is commutative, and is dual to the signed free associative algebra, which is cocommutative.

\subsection{The Involutions $\tau$ and $\tilde{\tau}$}

Here are various adjectives describing endomorphisms of a Hopf algebra, of which the last is new and convenient for this work.

Definition 3. Let $\mathcal{H}$ be a graded connected Hopf algebra, and $f: \mathcal{H} \rightarrow \mathcal{H}$ a function.

- $f$ is graded if $f\left(\mathcal{H}_{n}\right) \subseteq \mathcal{H}_{n}$; 
- $f$ is an involution if $f \circ f=\mathrm{id}$;

- $f$ is an algebra morphism if $f(w z)=f(w) f(z)$ for all $w, z \in \mathcal{H}$;

- $f$ is an algebra antimorphism if $f(w z)=f(z) f(w)$ for all $w, z \in \mathcal{H}$;

- $f$ is a coalgebra morphism if, whenever $\Delta(x)=\sum_{i} w_{i} \otimes z_{i}$, then $\Delta(f(x))=$ $\sum_{i} f\left(w_{i}\right) \otimes f\left(z_{i}\right)$

- $f$ is a coalgebra antimorphism if, whenever $\Delta(x)=\sum_{i} w_{i} \otimes z_{i}$, then $\Delta(f(x))=$ $\sum_{i} f\left(z_{i}\right) \otimes f\left(w_{i}\right)$

- $f$ is a Hopf morphism if it is both an algebra morphism and coalgebra morphism.

- $f$ is Hopf ambimorphism if it is either an algebra morphism or algebra antimorphism, and also either a coalgebra morphism or coalgebra antimorphism.

Let $\tau: \mathcal{H} \rightarrow \mathcal{H}$ denote an involution that is linear, graded and a Hopf ambimorphism. Given the definition of ambimorphism, there are $2 \times 2=4$ possible combinations for how $\tau$ interacts with the Hopf structure of $\mathcal{H}$, and some general results (e.g. Theorem 17 concerning the spectrum of associated descent operators) apply equally in all four cases. Where the four possibilities behave differently, we may use $\tau$ and $\tilde{\tau}$ to emphasise the differences, where $\tau$ usually includes the case of a Hopf morphism and perhaps also other cases.

Note that, if $\mathcal{H}$ is commutative, then an algebra morphism is also an algebra antimorphism; dually, if $\mathcal{H}$ is cocommutative, then a coalgebra morphism is also a coalgebra antimorphism.

Example 4. Recall the signed shuffle algebra of Example 1. Since this Hopf algebra is commutative, there are two possible types of Hopf-ambimorphism: we may let $\tau$ be an algebra morphism and coalgebra morphism, or we may let $\tilde{\tau}$ be an algebra morphism and coalgebra antimorphism. For the card-shuffling applications in this work, we focus on the involutions from [BB92]: let $\tau$ model the rotation of a deck by $180^{\circ}$ (where $\bar{i}$ denotes a rotated copy of card $i$ ), and let $\tilde{\tau}$ model the flipping of a deck (where $\bar{i}$ denotes card $i$ facing down). In terms of words, we define

$$
\begin{aligned}
\tau(i) & :=\bar{i} \\
\tau(\bar{i}) & :=i \\
\tau\left(w_{1} \ldots w_{n}\right) & :=\tau\left(w_{1}\right) \ldots \tau\left(w_{n}\right) \\
\tilde{\tau}(i) & :=\bar{i} \\
\tilde{\tau}(\bar{i}) & :=i \\
\tilde{\tau}\left(w_{1} \ldots w_{n}\right) & :=\tilde{\tau}\left(w_{n}\right) \ldots \tilde{\tau}\left(w_{1}\right)
\end{aligned}
$$

and extend linearly. For example, $\tau(3 \overline{1} 6)=\overline{3} 1 \overline{6}$ whilst $\tilde{\tau}(3 \overline{1} 6)=\overline{6} 1 \overline{3}$. The crucial difference between rotating and flipping a deck is that flipping reverses the top-to-bottom 
order of the cards while rotation does not - this is why rotation is a Hopf morphism but flipping is not.

Equations (2) and (3) can also be applied in the signed free associative algebra, then $\tau$ is a Hopf morphism, and $\tilde{\tau}$ is an algebra antimorphism and a coalgebra morphism. (These involutions are actually dual to the $\tau$ and $\tilde{\tau}$ defined by the same equations on the signed shuffle algebra.)

Further, $\tilde{\tau}$ is compatible with the quotient of the signed free associative algebra to the unsigned free associative algebra; in other words, we may define

$$
\tilde{\tau}\left(w_{1} \ldots w_{n}\right):=w_{n} \ldots w_{1}
$$

on the unsigned free associative algebra, which is an algebra antimorphism and a coalgebra morphism.

Below is another example that is interesting for future study.

Example 5. The paper [CP17] defines a Hopf algebra structure $C a m b^{*}$ on Cambrian trees, a type of planar binary tree where each internal node has either two upward edges and one downward edge, or two downward edges and one upward edge. Loosely speaking, the product assembles one tree on top of another (vertical), and the coproduct divides a tree into left and right (horizontal). Thus horizontal reflection is an algebra morphism and a coalgebra antimorphism; vertical reflection is an algebra antimorphism and coalgebra morphism; $180^{\circ}$-rotation is an algebra antimorphism and a coalgebra antimorphism. Indeed, $180^{\circ}$-rotation is a composition of the two reflections, and it is easy to check that composing two commuting involutive Hopf-ambimorphisms always yields another involutive Hopf-ambimorphism.

The following notation will be useful:

Definition 6. Let $V$ be a vector space and $\tau: V \rightarrow V$ be a linear map. Then the $\tau$-invariant part of $V$, written $V^{\tau}$, is the eigenspace of eigenvalue 1 . The $\tau$-negating part of $V$, written $V^{-\tau}$, is the eigenspace of eigenvalue -1 .

Please note the following easy linear algebra lemma:

Lemma 7. If $\tau$ is an involution on a vector space $V$, then $V=V^{\tau} \oplus V^{-\tau}$.

Proof. Clearly $V^{\tau}+V^{-\tau}$ is a direct sum since eigenspaces of different eigenvalues have trivial intersection. Given any $v \in V$, we have $v+\tau(v) \in V^{\tau}$ and $v-\tau(v) \in V^{-\tau}$, so $v=\frac{v+\tau(v)}{2}+\frac{v-\tau(v)}{2} \in V^{\tau} \oplus V^{-\tau}$.

To derive eigenvalues and eigenvectors, it will be important to consider the $\tau$-invariant and $\tau$-negating parts of another subspace.

Lemma 8. If $\mathcal{H}$ is a graded connected Hopf algebra and $\tau: \mathcal{H} \rightarrow \mathcal{H}$ is a coalgebra morphism or coalgebra antimorphism, then $\tau$ preserves the primitive subspace, i.e. $\tau(\operatorname{Prim}(\mathcal{H})) \subseteq \operatorname{Prim}(\mathcal{H})$ 
Proof. If $x \in \operatorname{Prim}(\mathcal{H})$, then $\Delta(x)=1 \otimes x+x \otimes 1$. If $\tau$ is a coalgebra morphism, then $\Delta(\tau(x))=\tau(1) \otimes \tau(x)+\tau(x) \otimes \tau(1)$. If $\tau$ is a coalgebra antimorphism, then $\Delta(\tau(x))=$ $\tau(x) \otimes \tau(1)+\tau(1) \otimes \tau(x)$. In both cases, $\tau(x)$ is primitive, by the equivalent characterisation just before Section 2.1 (applied linearly to the parts of $x$ in each degree).

By the same argument, $\tau$ preserves the "coradical filtration", which allows important associated graded operators to be defined in Lemma 21 below, for computing the eigenvalues of hyperoctahedral descent operators.

\subsection{Hyperoctahedral Descent Operators}

The elementary hyperoctahedral descent operators are indexed by signed and tilde-signed weak-compositions, where some parts are decorated with - or with $\simeq$ respectively. For example, $(\overline{2}, 4,0, \overline{2})$ is a signed weak-composition. We do not allow the two types of decorations to occur together in the same weak-composition. And, unlike in [MR95b], we also do not allow parts to have a tilde without a sign, since this does not correspond natually to an endomorphism of a Hopf algebra. These objects are also called bi-coloured compositions in [BW17].

Given a signed or tilde-signed weak-compositions $D=\left(d_{1}, \ldots, d_{l}\right)$, let $D^{+}$denote the weak-composition formed from forgetting the decorations in $D$. We may also use absolute value signs to denote this, e.g. $D^{+}=\left(\left|d_{1}\right|, \ldots,\left|d_{l}\right|\right)$. For example, $(\overline{2}, 4,0, \overline{2})^{+}=(2,4,0,2)$, as $|\overline{2}|=2$.

Definition 9. Let $\mathcal{H}$ be a graded Hopf algebra, and $\tau: \mathcal{H} \rightarrow \mathcal{H}$ be an involution that is linear, graded and a Hopf morphism. Let $D$ be a signed weak-composition. Then

- the signed refined coproduct $\Delta_{D(\tau)}$ is the composite: first apply the refined coproduct $\Delta_{D^{+}}$, then apply $\tau$ to the tensorands corresponding to the decorated parts.

- the elementary signed descent operators are the composites $m \circ \Delta_{D(\tau)}$.

- the signed descent operators are linear combinations of the $m \circ \Delta_{D(\tau)}$ over different signed weak-compositions $D$.

All three maps have tilde-signed analogues, by using tilde-signed weak-compositions, and an involution $\tilde{\tau}$ that is a Hopf ambimorphism but not a Hopf morphism. We refer to both families of operators as hyperoctahedral descent operators. In numerical examples, when $\tau$ is clear from the context, we often write $m \circ \Delta_{D}$ in place of $m \circ \Delta_{D(\tau)}$.

For example, $m \circ \Delta_{1, \tilde{2}}=m \circ(\mathrm{id} \otimes \tilde{\tau}) \circ \Delta_{1,2}$ is an elementary tilde-signed descent operator. In the shuffle algebra, using $\tilde{\tau}$ as defined in (3),

$$
m \circ \Delta_{1, \tilde{\overline{2}}}(3 \overline{1} 6)=m \circ(\mathrm{id} \otimes \tilde{\tau})(3 \otimes \overline{1} 6)=m(3 \otimes \overline{6} 1)=3 \overline{6} 1+\overline{6} 31+\overline{6} 13 .
$$

And $m \circ \Delta_{\overline{2}, 4, \overline{2}}+m \circ \Delta_{\overline{3}, 5}=m \circ(\tau \otimes \mathrm{id} \otimes \tau) \circ \Delta_{2,4,2}+m \circ(\tau \otimes \mathrm{id}) \circ \Delta_{3,5}$ is a signed descent operator. 
Note that the dual of $m \circ \Delta_{D(\tau)}$ is $m \circ \Delta_{D\left(\tau^{*}\right)}$ on the dual Hopf algebra, where $\tau^{*}$ : $\mathcal{H}^{*} \rightarrow \mathcal{H}^{*}$ denotes the linear-algebraic dual map. This will be important when considering right eigenfunctions of the associated Markov chains.

If $\mathcal{H}$ is commutative or cocommutative, then the vector space of signed descent operators (for a fixed $\tau$ ) is closed under composition, and is isomorphic to the MantaciReutenauer algebra. The vector space of tilde-signed descent operators is also closed under composition. The case where $\mathcal{H}$ is the signed free associative algebra and $\tau, \tilde{\tau}$ are as in (2) and (3) is part of Theorems 2.2, 3.8 and Corollary 5.3 of [MR95b]. Minor modifications suffice to extend the proof to the general case. Stating the composition formula requires the following concepts:

Definition 10. Let $D=\left(d_{1}, \ldots, d_{l}\right)$ and $D^{\prime}=\left(d_{1}^{\prime}, \ldots, d_{l^{\prime}}^{\prime}\right)$ be two signed weak-compositions or two tilde-signed weak-compositions. An $l \times l^{\prime}$ matrix $M=\left(M_{i, j}\right)$ of integers is called compatible with $D$ and $D^{\prime}$ (the order matters) if the following two conditions are satisfied:

i) The row sums of $M^{+}$are $D^{+}$, and the column sums of $M^{+}$are $\left(D^{\prime}\right)^{+}$, i.e. $\sum_{j=1}^{l^{\prime}}\left|M_{i, j}\right|$ $=\left|d_{i}\right|$ for each $i \in[1, l]$, and $\sum_{i=1}^{l}\left|M_{i, j}\right|=\left|d_{j}^{\prime}\right|$ for each $j \in\left[1, l^{\prime}\right]$.

ii) The sign of $M_{i, j}$ is the product of the signs of $d_{i}$ and $d_{j}^{\prime}$.

Let $\operatorname{Mat}\left(D, D^{\prime}\right)$ denote the set of matrices compatible with $D$ and $D^{\prime}$. For each $M \in$ $\operatorname{Mat}\left(D, D^{\prime}\right)$, let wcomp $(M)$ denote the signed weak-composition formed by reading left to right across each row from the top row to the bottom row

$$
\operatorname{wcomp}(M)=\left(M_{1,1}, M_{1,2}, \ldots, M_{1, l^{\prime}}, M_{2,1}, \ldots M_{2, l^{\prime}}, \ldots, M_{l, 1}, \ldots, M_{l, l^{\prime}}\right) .
$$

And let $\widetilde{w c o m p}_{D}(M)$ denote the tilde-signed weak-composition formed by reading each row from the top row to the bottom row, where the $i$ th row is read left to right if $d_{i}$ is positive, and read right to left if $d_{i}$ is negative (i.e. decorated).

Example 11. Let $D=(\overline{2}, \overline{4}, 1)$ and $D^{\prime}=(\overline{2}, 5)$. Then $\operatorname{Mat}\left(D, D^{\prime}\right)$ consists of the five matrices below:

$$
\begin{array}{llllllllll}
0 & \overline{2} & 1 & \overline{1} & 1 & \overline{1} & 2 & \overline{0} & 0 & \overline{2} \\
1 & \overline{3}, & 0 & \overline{4}, & 1 & \overline{3} & 0 & \overline{4} & 2 & \overline{2} . \\
\overline{1} & 0 & \overline{1} & 0 & \overline{0} & 1 & \overline{0} & 1 & \overline{0} & 1
\end{array}
$$

If $M$ is the first matrix in this list, then $\operatorname{wcomp}(M)=(0, \overline{2}, 1, \overline{3}, \overline{1}, 0)$.

Now take $D=(\tilde{\overline{2}}, \tilde{\overline{4}}, 1), D^{\prime}=(\tilde{\overline{2}}, 5)$ and

$$
M=\begin{array}{cc}
0 & \overline{\overline{2}} \\
1 & \overline{\overline{3}} \\
\tilde{\overline{1}} & 0
\end{array} \in \operatorname{Mat}\left(D, D^{\prime}\right)
$$

Then $\widetilde{\operatorname{comp}}_{D}(M)=(\tilde{\overline{2}}, 0, \tilde{\overline{3}}, 1, \tilde{\overline{1}}, 0)$.

Proposition 12. Under the setup of Definitions 9 and 10: 
i) if $\mathcal{H}$ is commutative, then

$$
\begin{aligned}
& \left(m \circ \Delta_{D(\tau)}\right) \circ\left(m \circ \Delta_{D^{\prime}(\tau)}\right)=\sum_{M \in \operatorname{Mat}\left(D^{\prime}, D\right)} m \circ \Delta_{\mathrm{wcomp}(M)} ; \\
& \left(m \circ \Delta_{D(\tilde{\tau})}\right) \circ\left(m \circ \Delta_{D^{\prime}(\tilde{\tau})}\right)=\sum_{M \in \operatorname{Mat}\left(D^{\prime}, D\right)} m \circ \Delta_{\mathrm{wcomp}_{D^{\prime}}(M)} ;
\end{aligned}
$$

ii) if $\mathcal{H}$ is cocommutative, then

$$
\begin{aligned}
& \left(m \circ \Delta_{D(\tau)}\right) \circ\left(m \circ \Delta_{D^{\prime}(\tau)}\right)=\sum_{M \in \operatorname{Mat}\left(D, D^{\prime}\right)} m \circ \Delta_{\mathrm{wcomp}(M)} \\
& \left(m \circ \Delta_{D(\tilde{\tau})}\right) \circ\left(m \circ \Delta_{D^{\prime}(\tilde{\tau})}\right)=\sum_{M \in \operatorname{Mat}\left(D, D^{\prime}\right)} m \circ \Delta_{\mathrm{wcomp}_{D}(M)} .
\end{aligned}
$$

Composition formulas for more complex hyperoctahedral descent operators can be obtained by taking linear combinations of the above. For example, [DFH13, Th. 4.2] treats the composition of $\sum_{D} m \circ \Delta_{D(\tilde{\tau})}$ on the unsigned shuffle algebra, where the sum ranges over all tilde-signed weak-compositions of $a$ parts and with a fixed sequence of $a$ signs. As another example, Proposition 24 will derive the composition formula for hyperoctahedral-riffle-shuffles.

Proof. We follow the argument of [MR95b]. Let $D=\left(d_{1}, \ldots, d_{l}\right)$ and $D^{\prime}=\left(d_{1}^{\prime}, \ldots, d_{l^{\prime}}^{\prime}\right)$ be signed weak-compositions. For $i \in[1, l]$ and $j \in\left[1, l^{\prime}\right]$, define

$$
\begin{gathered}
f_{i}= \begin{cases}\text { id } & \text { if } d_{i} \geqslant 0 ; \\
\tau & \text { if } d_{i}<0 ;\end{cases} \\
g_{j}= \begin{cases}\text { id } & \text { if } d_{j}^{\prime} \geqslant 0 ; \\
\tau & \text { if } d_{j}^{\prime}<0\end{cases}
\end{gathered}
$$

Then

$$
\begin{aligned}
& \left(m \circ \Delta_{D(\tau)}\right) \circ\left(m \circ \Delta_{D^{\prime}(\tau)}\right) \\
= & \left(m \circ\left(f_{1} \otimes \cdots \otimes f_{l}\right) \circ \Delta_{D^{+}}\right) \circ\left(m \circ\left(g_{1} \otimes \cdots \otimes g_{l^{\prime}}\right) \circ \Delta_{D^{\prime+}}\right) \\
= & \sum m \circ\left(f_{1} \otimes \cdots \otimes f_{l}\right) \circ m \circ \sigma \circ\left(\Delta_{D_{1}} \otimes \cdots \otimes \Delta_{D_{l^{\prime}}}\right) \circ\left(g_{1} \otimes \cdots \otimes g_{l^{\prime}}\right) \circ \Delta_{D^{\prime+}}
\end{aligned}
$$

by the compatibility axiom between $m$ and $\Delta$. Here, the sum is over all $l^{\prime}$-tuples $\left(D_{1}, \ldots, D_{l^{\prime}}\right)$ of weak-compositions of $\left|d_{1}^{\prime}\right|, \ldots,\left|d_{l^{\prime}}^{\prime}\right|$ respectively, that satisfy $\left(D_{1}\right)_{i}+\cdots+$ $\left(D_{l^{\prime}}\right)_{i}=\left|d_{i}\right|$. And $\sigma: \mathcal{H}^{\otimes l l^{\prime}} \rightarrow \mathcal{H}^{\otimes l l^{\prime}}$ is a permutation of the tensorands corresponding to reading a matrix in columns instead of rows:

$$
\begin{aligned}
\sigma\left(x_{1} \otimes \cdots \otimes x_{l l^{\prime}}\right)= & \left(x_{1} \otimes x_{l+1} \otimes \cdots \otimes x_{l\left(l^{\prime}-1\right)+1}\right) \\
& \otimes\left(x_{2} \otimes \cdots \otimes x_{l\left(l^{\prime}-1\right)+2}\right) \otimes \ldots \\
& \otimes\left(x_{l^{\prime}} \otimes \cdots \otimes x_{l l^{\prime}}\right) .
\end{aligned}
$$


Because $g_{j}$ are coalgebra morphisms, and $f_{i}$ are algebra morphisms, the above is equal to

$$
\begin{aligned}
& \sum m \circ\left(f_{1}^{\otimes l^{\prime}} \otimes \cdots \otimes f_{l}^{\otimes l^{\prime}}\right) \circ \sigma \circ\left(g_{1}^{\otimes l} \otimes \cdots \otimes g_{l^{\prime}}^{\otimes l}\right) \circ\left(\Delta_{D_{1}} \otimes \cdots \otimes \Delta_{D_{l^{\prime}}}\right) \circ \Delta_{D^{\prime+}} \\
= & \sum m \circ\left(f_{1}^{\otimes l^{\prime}} \otimes \cdots \otimes f_{l}^{\otimes l^{\prime}}\right) \circ \sigma \circ\left(g_{1}^{\otimes l} \otimes \cdots \otimes g_{l^{\prime}}^{\otimes l}\right) \circ \Delta_{D_{1} \cdots D_{l^{\prime}}}
\end{aligned}
$$

where $D_{1} \cdots D_{l^{\prime}}$ is a concatenation of weak-compositions. This last equality follows from coassociativity.

If $\mathcal{H}$ is commutative, then $m \circ \sigma=m$, so (6) is equal to

$$
\begin{aligned}
& \sum m \circ \sigma \circ\left(\left(f_{1} \otimes \cdots \otimes f_{l}\right)^{\otimes l^{\prime}}\right) \circ\left(g_{1}^{\otimes l} \otimes \cdots \otimes g_{l^{\prime}}^{\otimes l}\right) \circ \Delta_{D_{1} \cdots \cdots D_{l^{\prime}}} \\
= & \sum m \circ\left(f_{1} \circ g_{1} \otimes f_{2} \circ g_{1} \otimes \cdots \otimes f_{l} \circ g_{1}\right. \\
& \otimes f_{1} \circ g_{2} \otimes \cdots \otimes f_{l} \circ g_{2} \otimes \cdots \\
& \left.\otimes f_{1} \circ g_{l^{\prime}} \otimes \cdots \otimes f_{l} \circ g_{l^{\prime}}\right) \circ \Delta_{D_{1} \cdots \cdots D_{l^{\prime}}} \\
= & \sum_{M \in \operatorname{Mat}\left(D^{\prime}, D\right)} m \circ \Delta_{\mathrm{wcomp}(M) .}
\end{aligned}
$$

If $\mathcal{H}$ is cocommutative, then

$$
\sigma \circ \Delta_{D_{1} \ldots \ldots D_{l^{\prime}}}=\Delta_{\left(\left(D_{1}\right)_{1},\left(D_{2}\right)_{1}, \ldots,\left(D_{l^{\prime}}\right)_{1},\left(D_{1}\right)_{2}, \ldots,\left(D_{l^{\prime}}\right)_{2}, \ldots,\left(D_{l}\right)_{1}, \ldots,\left(D_{l^{\prime}}\right)_{l}\right.},
$$

so (6) is equal to

$$
\begin{aligned}
& \sum m \circ\left(f_{1}^{\otimes l^{\prime}} \otimes \cdots \otimes f_{l}^{\otimes l^{\prime}}\right) \circ\left(\left(g_{1} \otimes \cdots \otimes g_{l^{\prime}}\right)^{\otimes l}\right) \circ \sigma \circ \Delta_{D_{1} \cdots \cdots D_{l^{\prime}}} \\
= & \sum_{M \in \operatorname{Mat}\left(D, D^{\prime}\right)} m \circ\left(f_{1} \circ g_{1} \otimes f_{1} \circ g_{2} \otimes \cdots \otimes f_{l} \circ g_{l^{\prime}} \circ\right. \\
& \otimes f_{2} \circ g_{1} \otimes \cdots \otimes f_{2} \circ g_{l^{\prime}} \otimes \ldots \\
& \left.\otimes f_{l} \circ g_{1} \otimes \cdots \otimes f_{l} \circ g_{l^{\prime}}\right) \circ \Delta_{\mathrm{wcomp}(M)^{+}} \\
= & \sum_{M \in \operatorname{Mat}\left(D, D^{\prime}\right)} m \circ \Delta_{\mathrm{wcomp}(M) .}
\end{aligned}
$$

Now let $D, D^{\prime}$ be tilde-signed weak-compositions, and let $\tilde{\tau}$ be an algebra morphism and a coalgebra antimorphism on a commutative $\mathcal{H}$. Performing the analogous calculations, the only change is that, in (5), each $\Delta_{D_{j}}$ must be calculated using the reverse weak-composition of $D_{j}$ if $d_{j}^{\prime}$ is tilde-signed. This change persists through to (7), where additionally $f_{j} \circ g_{1} \otimes \cdots \otimes f_{j} \circ g_{l^{\prime}}$ must be replaced by $f_{j} \circ g_{l^{\prime}} \otimes \cdots \otimes f_{j} \circ g_{1}$ whenever $d_{j}^{\prime}$ is tilde-signed. Thus the result is a sum of $m \circ \Delta_{\text {wcomp }_{D}(M)}$ terms instead of $m \circ \Delta_{\text {wcomp }(M)}$. The case is similar when $\tilde{\tau}$ is an algebra antimorphism and a coalgebra morphism on a cocommutative $\mathcal{H}$. 


\section{$2.3 \quad$ Eigenvalues}

Just as the eigenvalues of type A descent operators are indexed by partitions, the eigenvalues of hyperoctahedral descent operators are indexed by double-partitions of $n$ - that is, pairs of partitions $\lambda, \bar{\lambda}$ with $\sum_{i} \lambda_{i}+\sum_{i} \bar{\lambda}_{i}=n$. (It's more convenient here to view partitions as multisets of integers, not arranged in any particular order.) The values of these eigenvalues themselves are again in terms of set-compositions, now of signed integers, compatible with $\lambda, \bar{\lambda}, D$ as defined below.

Definition 13. Given a set $S$, a set-composition of $S$ is a sequence $B_{1}, \ldots, B_{l}$ of (possibly empty) disjoint subsets of $S$ with $B_{1} \amalg \cdots \amalg B_{l}=S$. A set-composition is usually written as $B=B_{1}|\ldots| B_{l}$, and the $B_{i}$ are called blocks.

Definition 14. Fix a double-partition $\lambda, \bar{\lambda}$ of $n$.

- Given a weak-composition $D$ (not signed) of $n$, a set-composition $B_{1} \amalg \cdots \amalg B_{l(D)}$ of $\{1,2, \ldots, l(\lambda)\} \cup\{\overline{1}, \overline{2}, \ldots, \overline{l(\bar{\lambda})}\}$ is called compatible with $\lambda, \bar{\lambda}, D$ if, for each $i \leqslant l(D)$, we have $\sum_{j \in B_{i}} \lambda_{j}+\sum_{\bar{j} \in B_{i}} \bar{\lambda}_{j}=d_{i}$.

- Given a signed or tilde-signed weak-composition $D$, let $\beta_{\lambda, \bar{\lambda}}^{D}$ denote a signed count of set-compositions compatible with $\lambda, \bar{\lambda}, D^{+}$. The sign of each set-composition is the parity of signed integers within a block corresponding to a decorated part of D.

Example 15. Let $n=10$ and $D=(\overline{5}, 4,1)$. The set-compositions compatible with the double-partition $\lambda=(4,2), \bar{\lambda}=(2,1,1), D^{+}$are $1 \overline{2}|\overline{1} 2| \overline{3}$ (corresponding to $D^{+}=$ $\left.\left(\lambda_{1}+\bar{\lambda}_{2}, \bar{\lambda}_{1}+\lambda_{2}, \bar{\lambda}_{3}\right)\right), 1 \overline{3}|\overline{1} 2| \overline{2}, \overline{1} 2 \overline{2}|1| \overline{3}, \overline{1} 2 \overline{3}|1| \overline{2}$. Since $D$ is decorated only in the first part, the sign of a compatible set-composition is -1 if there are an odd number of signed integers in the first block, as in $1 \overline{2}|\overline{1} 2| \overline{3}$ and $1 \overline{3}|\overline{1} 2| \overline{2}$. For $\overline{1} 2 \overline{2}|1| \overline{3}$ and $\overline{1} 2 \overline{3}|1| \overline{2}$, the sign is +1 as their first blocks contain an even number of signed integers. Thus $\beta_{\lambda, \bar{\lambda}}^{D}=-1-1+1+1=0$ in this example.

To describe the multiplicities, we follow the notation of [AS05]:

Notation 16. Given a partition $\lambda$, let $m_{i}(\lambda)$ denote the number of parts of size $i$. Given also a sequence $\boldsymbol{b}=\left(b_{i}\right)_{i \geqslant 1}$, let $\left(\begin{array}{c}\boldsymbol{b} \\ \lambda\end{array}\right)$ be the number of ways to choose, for each $i, m_{i}$ objects amongst $b_{i}$, possibly with repetition. In other words,

$$
\left(\begin{array}{c}
\boldsymbol{b} \\
\lambda
\end{array}\right)=\left(\begin{array}{c}
b_{1}+m_{1}-1 \\
m_{1}
\end{array}\right)\left(\begin{array}{c}
b_{1}+m_{2}-1 \\
m_{2}
\end{array}\right) \ldots,
$$

or equivalently $\left(\begin{array}{l}\boldsymbol{b} \\ \lambda\end{array}\right)$ is the coefficient of $x_{\lambda}:=x_{\lambda_{1}} \ldots x_{\lambda_{l(\lambda)}}$ in the generating function $\prod_{i}\left(1-x_{i}\right)^{-b_{i}}$.

Point ii. of the Theorem below will apply this to two sequences $\boldsymbol{b}$ and $\overline{\boldsymbol{b}}$, which are the graded dimensions of the $\tau$-invariant and $\tau$-negating subspaces of the primitive subspace of $\mathcal{H}$, if $\mathcal{H}$ is cocommutative (and of a related subspace if $\mathcal{H}$ is not cocommutative, which will be explained in the proof). It is an interesting open problem to express the numbers $\left(\begin{array}{l}\boldsymbol{b} \\ \lambda\end{array}\right)\left(\begin{array}{l}\bar{b} \\ \bar{\lambda}\end{array}\right)$ in terms of double-partitions, bi-tableaux, or other known combinatorial objects. 
Theorem 17. Let $\mathcal{H}=\bigoplus \mathcal{H}_{n}$ be a graded connected Hopf algebra over $\mathbb{R}, \tau: \mathcal{H} \rightarrow \mathcal{H}$ a linear graded involution that is a Hopf-ambimorphism. Let $D$ be a signed or tilde-signed weak-composition of $n$.

i) The eigenvalues of the associated hyperoctahedral descent operator $m \circ \Delta_{D(\tau)}: \mathcal{H}_{n} \rightarrow$ $\mathcal{H}_{n}$ are $\beta_{\lambda, \bar{\lambda}}^{D}$, as $\lambda, \bar{\lambda}$ ranges over all double-partitions of $n$.

ii) The multiplicity of the eigenvalue $\beta_{\lambda, \bar{\lambda}}^{D}$ is $\left(\begin{array}{l}\boldsymbol{b} \\ \lambda\end{array}\right)\left(\begin{array}{l}\overline{\boldsymbol{b}} \\ \bar{\lambda}\end{array}\right)$, where the sequences $\boldsymbol{b}$ and $\overline{\boldsymbol{b}}$ satisfy the identities

$$
\begin{aligned}
\sum_{n} \operatorname{dim} \mathcal{H}_{n} x^{n} & =\prod_{i}\left(1-x^{i}\right)^{-b_{i}-\overline{b_{i}}} \\
\sum_{n}\left(\operatorname{dim} \mathcal{H}_{n}^{\tau}-\operatorname{dim} \mathcal{H}_{n}^{-\tau}\right) x^{n} & =\prod_{i}\left(1-x^{i}\right)^{-b_{i}} \prod_{i}\left(1+x^{i}\right)^{-\bar{b}_{i}} .
\end{aligned}
$$

iii) Furthermore, if $D_{1}, \ldots, D_{r}$ are signed weak-compositions of $n$, or are tilde-signed weak-compositions of $n$, and $a_{1}, \ldots, a_{r} \in \mathbb{R}$, then the eigenvalues of $\sum_{i=1}^{r} a_{i} m \circ$ $\Delta_{D_{i}(\tau)}: \mathcal{H}_{n} \rightarrow \mathcal{H}_{n}$ are $\sum_{i=1}^{r} a_{i} \beta_{\lambda, \bar{\lambda}}^{D_{i}}$, with multiplicities as above.

The complicated expressions for the eigenvalues and multiplicities simplify somewhat for specific operators - see Examples 18 to 20, Proposition 25, and Section 5.4 - in the latter, the multiplicities for card-shuffling flipping every other pile are interpreted as hyperoctahedral analogues of Stirling numbers.

Note that, if $\tau=\mathrm{id}$, then the theorem recovers the type A case [Pan18, Th. 3.5], as all relevant set-compositions have positive sign. For other $\tau$, as long as its action on $\mathcal{H}$ gives the generic case where $b_{i}, \bar{b}_{i}$ are all non-zero, the eigenvalues are the same regardless of $\tau$; only the multiplicities depend on $\tau$. (If some $b_{i}$ or $\bar{b}_{i}$ is zero, then some of the generic eigenvalues might not be achieved.)

Example 18. We compute the eigenvalues of $m \circ \Delta_{(\overline{1}, n-1)}$. (Under the correspondence of Theorem 34, this operator models the shuffle that removes the top card from a deck of $n$ cards, rotates it by 180 degrees, then reinserts it in a uniformly chosen position.) For a double-partition $\lambda, \bar{\lambda}$, the parts of size 1 in $\lambda$ or $\bar{\lambda}$ are in bijection with set-compositions compatible with $\lambda, \bar{\lambda},(1, n-1)$, by considering the first block. A part of size 1 in $\lambda$ induces a positive sign, whereas a part from $\bar{\lambda}$ induces a negative sign. Hence, if $1(\lambda)$ denotes the number of parts of size 1 in $\lambda$, we have $\beta_{\lambda, \bar{\lambda}}^{(\overline{1}, n-1)}=1(\lambda)-1(\bar{\lambda})$. Since $1(\lambda)$ and $1(\bar{\lambda})$ can take any two values in $\{0,1, \ldots, n\}$ that sum to $\{0,1, \ldots, n-2\} \cup\{n\}$, the generic eigenvalues are $\{-n\} \cup\{-n+2,-n+1, \ldots, n-2\} \cup\{n\}$. (To obtain the eigenvalues of the card shuffle, we should divide by $n$, i.e. they are $\frac{k}{n}$ for $k \in\{-n\} \cup\{-n+2,-n+1, \ldots, n-2\} \cup\{n\}$.)

Example 19. We compute the eigenvalues of $m \circ(\tilde{\tau} \otimes \mathrm{id}) \circ \Delta=\sum_{i=0}^{n} m \circ \Delta_{(\tilde{\bar{i}}, n-i)}$; this operator is BRiffle $\tilde{\tau}_{2}^{-}$in the notation of Section 3, and represents the shuffle that cuts the deck binomially, flips the first pile upside-down, then interleave the piles together. The paper [PP98] previously computed these eigenvalues for a related inverse shuffle, that corresponds to the case of the unsigned free associative algebra, with $\tilde{\tau}$ as in (4). 
Fix a double-partition $\lambda, \bar{\lambda}$. Every set-composition of $\{1,2, \ldots, l(\lambda)\} \cup\{\overline{1}, \overline{2}, \ldots, \overline{l(\bar{\lambda})}\}$ into two blocks is compatible with $\lambda, \bar{\lambda},(\tilde{\bar{i}}, n-i)$ for exactly one $i$, namely $i=\sum_{j \in B_{1}} \lambda_{j}+$ $\sum_{\bar{j} \in B_{1}} \bar{\lambda}_{j}$. The associated sign is the parity of signed integers in the first block. If $\bar{\lambda} \neq \varnothing$, then there is a sign-reversing involution on these set-compositions: if $\overline{1} \in B_{1}$, move it to $B_{2}$, and if $\overline{1} \in B_{2}$, move it to $B_{1}$. Hence the signed count $\sum_{i=0}^{n} \beta_{\lambda, \bar{\lambda}}^{(\tilde{\bar{i}}, n-i)}$ is 0 . If $\bar{\lambda}=\varnothing$, then the associated eigenvalue $\sum_{i=0}^{n} \beta_{\lambda, \bar{\lambda}}^{(\tilde{\bar{i}}, n-i)}$ is the number of set-compositions of $\{1,2, \ldots, l(\lambda)\}$ into two blocks, which is $2^{l(\lambda)}$. Since $l(\lambda)$ can take any value in $\{1,2, \ldots, n\}$ when $\bar{\lambda}=\varnothing$, the generic eigenvalues are $2,4, \ldots 2^{n}$ and 0 . (The eigenvalues of the card shuffle require dividing by $2^{n}$, i.e. they are $2^{-k}$ for $k \in\{0,1, \ldots, n-1\}$, and 0 .)

Example 20. Consider the following new type of card shuffle: deconcatenate the deck into two piles according to the binomial distribution, then flip a coin for each pile to decide whether or not to flip it before interleaving the piles together. According to Theorem 34, the corresponding hyperoctahedral descent operator (up to scaling by $2^{n+2}$ ) is $\sum m \circ \Delta_{D}$, summing over all tilde-signed weak-compositions $D$ of $n$ with two parts. So its eigenvalues are $\sum_{i=0}^{n} \beta_{\lambda, \bar{\lambda}}^{(i, n-i)}+\beta_{\lambda, \bar{\lambda}}^{(\bar{i}, n-i)}+\beta_{\lambda, \bar{\lambda}}^{(i, \overline{n-i})}+\beta_{\lambda, \bar{\lambda}}^{(\bar{i}, \overline{n-i})}$. Each of the $2^{l(\lambda)+l(\bar{\lambda})}$ set-compositions of $\{1,2, \ldots, l(\lambda)\} \cup\{\overline{1}, \overline{2}, \ldots, \overline{l(\bar{\lambda})}\}$ into two blocks contributes +1 to $\beta_{\lambda, \bar{\lambda}}^{(i, n-i)}$ and $(-1)^{l(\bar{\lambda})}$ to $\beta_{\lambda, \bar{\lambda}}^{(\bar{i}, \overline{n-i})}$, where $i=\sum_{j \in B_{1}} \lambda_{j}+\sum_{\bar{j} \in B_{1}} \bar{\lambda}_{j}$. And, by a sign-reversing involution as in Example $19, \sum_{i=0}^{n} \beta_{\lambda, \bar{\lambda}}^{(\bar{i}, n-i)}=\sum_{i=0}^{n} \beta_{\lambda, \bar{\lambda}}^{(i, \overline{n-i})}=0$ if $\bar{\lambda} \neq \varnothing$, and else is $2^{l(\lambda)}$. Hence the eigenvalues are

$$
\sum \beta_{\lambda, \bar{\lambda}}^{D}= \begin{cases}2^{l(\lambda)+2} & \text { if } l(\bar{\lambda})=0 \\ 2^{l(\lambda)+l(\bar{\lambda})+1} & \text { if } l(\bar{\lambda})>0 \text { is even; } \\ 0 & \text { if } l(\bar{\lambda}) \text { is odd }\end{cases}
$$

So the generic eigenvalues are $8,16, \ldots, 2^{n+2}$ and 0 , and for the card shuffle they are $2^{-k}$ for $k \in\{0,1, \ldots, n-1\}$, and 0 .

Remarks.

1. Note that, as for the type A case, the eigenvalues $\beta_{\lambda, \bar{\lambda}}^{D}$ may coincide for different choices of $\lambda$ and $\bar{\lambda}$. For example, if $D=(\overline{1}, n-1)$, then $\beta_{\lambda, \bar{\lambda}}^{D}=0$ whenever neither $\lambda$ nor $\bar{\lambda}$ has a part of size 1 .

2. Unlike the type A case, the present theorem does not claim that $m \circ \Delta_{D(\tau)}$ is diagonalisable on commutative or cocommutative Hopf algebras. Sage computations show that this is in fact false. The diagonalisability proof in [Pan18, Sec. 3.2] for type A descent operators does not extend to hyperoctahedral descent operators because the matrices involved there will gain negative entries when extended to type B, so Perron-Frobenius will not apply.

3. The proof below can generalise to give the spectrum of convolution products $\mathbf{T}_{1} *$ $\cdots * \mathbf{T}_{l}:=m \circ\left(\mathbf{T}_{1} \otimes \cdots \otimes \mathbf{T}_{l}\right) \circ \Delta^{[l]}$, where the $\mathbf{T}_{i}$ are simultaneously diagonalisable 
graded Hopf ambimorphisms. Then the eigenvalues $\beta_{\lambda}$ would be a weighted count of set-compositions, where the weights are the appropriate products of eigenvalues of the $\mathbf{T}_{i}$.

The two main ideas of the proof, as with [AL15, Th. 3] and [Pan18, Th. 3.5], are:

- reduce to the cocommutative case by working in $(\operatorname{gr} \mathcal{H})^{*}$, the dual of the associated graded Hopf algebra with respect to the coradical filtration (see [AL15, Sec. 1.3] for the definitions);

- examine the action of $\operatorname{gr}\left(m \circ \Delta_{D(\tau)}\right)^{*}$ on a Poincare-Birkhoff-Witt basis, i.e. on products of primitive elements.

The first part requires the following lemma:

Lemma 21. Under the conditions of Theorem 17,

i) The dual associated graded map is

$$
\left(\operatorname{gr}\left(m \circ \Delta_{D(\tau)}: \mathcal{H}_{n} \rightarrow \mathcal{H}_{n}\right)\right)^{*}=m \circ \Delta_{D\left((\operatorname{gr} \tau)^{*}\right)}:(\operatorname{gr} \mathcal{H})_{n}^{*} \rightarrow(\operatorname{gr} \mathcal{H})_{n}^{*} .
$$

ii) $(\operatorname{gr} \tau)^{*}$ is an involution.

iii) The dimensions of the fixed subspaces are related by $\operatorname{dim} \mathcal{H}_{n}^{\tau}=\operatorname{dim}\left(\operatorname{gr} \mathcal{H}^{*}\right)_{n}^{(\operatorname{gr} \tau)^{*}}$ and $\operatorname{dim} \mathcal{H}_{n}^{-\tau}=\operatorname{dim}\left(\operatorname{gr} \mathcal{H}^{*}\right)_{n}^{-(\operatorname{gr} \tau)^{*}}$.

iv) $(\operatorname{gr} \tau)^{*}$ is a Hopf ambimorphism.

Proof.

i) Since $\tau$ is a coalgebra morphism or antimorphism, $\tau$ preserves the coradical filtration. Hence gr $\tau: \operatorname{gr} \mathcal{H} \rightarrow \operatorname{gr} \mathcal{H}$ is well-defined. Then, taking the associated graded map is functorial and so preserves convolution products. The same is true for dualising.

ii) Taking the associated graded map is functorial and so preserves compositions. Hence $\tau \circ \tau=\mathrm{id}$ means $(\operatorname{gr} \tau) \circ(\operatorname{gr} \tau)=\mathrm{id}$, and dualising this shows that $(\operatorname{gr} \tau)^{*}$ is an involution.

iii) As noted in [AL15], $\tau$ and $\operatorname{gr}(\tau)$ have the same spectrum, and so does $(\operatorname{gr} \tau)^{*}$. Since $\tau$ and $(\operatorname{gr} \tau)^{*}$ are involutions, they are diagonalisable, and so their spectrum determines their eigenspace dimensions.

iv) It follows from the definition of the Hopf structure on $\operatorname{gr}(\mathcal{H})$ that, if $\tau$ is an algebra morphism (resp. antimorphism), then so is $\operatorname{gr}(\tau)$, and then $(\operatorname{gr} \tau)^{*}$ is a coalgebra morphism (resp. antimorphism). Similarly, if $\tau$ is a coalgebra morphism (resp. antimorphism), then $(\operatorname{gr} \tau)^{*}$ is an algebra morphism (resp. antimorphism). 
Any coradical-filtration preserving map and its dual associated graded map have the same spectrum, and $(\mathrm{gr} \mathcal{H})^{*}$ is cocommutative [Swe69, Th. 11.2.5.a][AS05, Prop. 1.6]. So the Lemma above reduces the proof to the case when $\mathcal{H}$ is cocommutative, where its structure is well-understood. Indeed, by the Cartier-Milnor-Moore theorem [Car07, Th. 3.8.1], a graded connected cocommutative Hopf algebra $\mathcal{H}$ is the universal enveloping algebra of its subspace of primitives. Consequently, $\mathcal{H}$ has a Poincare-BirkhoffWitt (PBW) basis: if $(\mathcal{P}, \preceq)$ is an ordered basis of the primitive subspace of $\mathcal{H}$, then $\left\{p_{1} \ldots p_{k} \mid k \in \mathbb{N}, p_{1} \preceq \ldots \preceq p_{k} \in \mathcal{P}\right\}$ is a basis of $\mathcal{H}$. The basis element $p_{1} \ldots p_{k}$ has length $k$. The key to the proof is the action of hyperoctahedral descent operators on this PBW basis, as stated in the following Triangularity Lemma (a substitute for the Symmetrisation Lemma of [Pan18, Lem. 3.8, 3.9]).

Notation 22. Let $\mathcal{H}$ be a graded connected Hopf algebra that is cocommutative, and let $\tau: \mathcal{H} \rightarrow \mathcal{H}$ be a linear graded involution that is a Hopf-ambimorphism. Consider $(\operatorname{Prim} \mathcal{H})^{\tau}$ and $(\operatorname{Prim} \mathcal{H})^{-\tau}$, which by Definition 6 are eigenspaces of $\tau$ of eigenvalue 1 and -1 respectively; let $(\mathcal{P}, \preceq)$ and $(\overline{\mathcal{P}}, \preceq)$ be respectively the ordered bases of these two subspaces. Then $\mathcal{P} \cup \overline{\mathcal{P}}$, with the "concatenation" order of $p \preceq \bar{p}$ for all $p \in \mathcal{P}$, $\bar{p} \in \overline{\mathcal{P}}$, is an ordered basis of Prim $\mathcal{H}$, and thus can be used to construct a PBW basis of $\mathcal{H}$. Let $p_{1}, \ldots, p_{k} \in \mathcal{P}, \bar{p}_{1}, \ldots, \bar{p}_{\bar{k}} \in \overline{\mathcal{P}}$ with $p_{1} \preceq \cdots \preceq p_{k}, \bar{p}_{1} \preceq \cdots \preceq \bar{p}_{\bar{k}}$, and let $\lambda=\left(\operatorname{deg} p_{1}, \ldots, \operatorname{deg} p_{k}\right), \bar{\lambda}=\left(\operatorname{deg} \bar{p}_{1}, \ldots, \operatorname{deg} \bar{p}_{\bar{k}}\right)$.

Lemma 23 (Triangularity Lemma). Under the setup of Notation 22:

$$
\begin{aligned}
m \circ \Delta_{D(\tau)}\left(p_{1} \ldots p_{k} \bar{p}_{1} \ldots \bar{p}_{\bar{k}}\right)= & \beta_{\lambda, \bar{\lambda}}^{D} p_{1} \ldots p_{k} \bar{p}_{1} \ldots \bar{p}_{\bar{k}} \\
& +P B W \text {-basis elements of length less than } k+\bar{k} .
\end{aligned}
$$

Consequently, relative to this PBW basis, the matrix for $m \circ \Delta_{D(\tau)}$ is triangular with $\beta_{\lambda, \bar{\lambda}}^{D}$ as its diagonal entries, and hence $\beta_{\lambda, \bar{\lambda}}^{D}$ are the eigenvalues.

Proof. By coassociativity,

$$
\Delta_{D^{+}}\left(p_{1} \ldots p_{k} \bar{p}_{1} \ldots \bar{p}_{\bar{k}}\right)=\sum_{B}\left(\prod_{i \in B_{1}} p_{i} \prod_{\bar{i} \in B_{1}} \bar{p}_{\bar{i}}\right) \otimes \cdots \otimes\left(\prod_{i \in B_{l(D)}} p_{i} \prod_{\bar{i} \in B_{l(D)}} \bar{p}_{\bar{i}}\right),
$$

where the sum is over all set-compositions $B$ compatible with $\lambda, \bar{\lambda}, D$. Hence

$$
m \circ \Delta_{D(\tau)}\left(p_{1} \ldots p_{k} \bar{p}_{1} \ldots \bar{p}_{\bar{k}}\right)=\sum_{B_{1}, \ldots, B_{l(D)}} \pm\left(\prod_{i \in B_{1}} p_{i} \prod_{\bar{i} \in B_{1}} \bar{p}_{\bar{i}}\right) \ldots\left(\prod_{i \in B_{l(D)}} p_{i} \prod_{\bar{i} \in B_{l(D)}} \bar{p}_{\bar{i}}\right)
$$

where the sign for each summand is the parity of elements of $\overline{\mathcal{P}}$ in decorated parts. (If $\tau$ is an algebra antimorphism, then for each decorated part $d_{i}$, the products in the $i$ th bracket above are taken in the reversed order.) Each summand is a product of $p_{1}, \ldots, p_{k}, \bar{p}_{1} \ldots, \bar{p}_{\bar{k}}$ in some order, so the PBW straightening algorithm [Kna02, Lem. III.3.9] rewrites each 
summand (excluding the sign) as $p_{1} \ldots p_{k} \bar{p}_{1} \ldots \bar{p}_{\bar{k}}+$ terms of length less than $k+\bar{k}$. Thus the coefficient of the highest length term $p_{1} \ldots p_{k} \bar{p}_{1} \ldots \bar{p}_{\bar{k}}$ in $m \circ \Delta_{D(\tau)}\left(p_{1} \ldots p_{k} \bar{p}_{1} \ldots \bar{p}_{\bar{k}}\right)$ is the signed number of summands, i.e. the signed number of set-compositions compatible with $\lambda, \bar{\lambda}, D$.

To continue the proof of Theorem 17: according to Lemma 23, the matrices of $m \circ \Delta_{D(\tau)}$ are simultaneously triangularisable with $\beta_{\lambda, \bar{\lambda}}^{D}$ on the diagonal, hence the eigenvalues of sums of $m \circ \Delta_{D(\tau)}$ are sums of these diagonal entries. The multiplicity of $\beta_{\lambda, \bar{\lambda}}^{D}$ is the number of multiset pairs $\left\{p_{1}, \ldots, p_{k}\right\} \subseteq \mathcal{P},\left\{\bar{p}_{1} \ldots, \bar{p}_{\bar{k}}\right\} \subseteq \overline{\mathcal{P}}$ whose degrees are given by $\lambda, \bar{\lambda}$. If $b_{i}=\operatorname{dim}(\operatorname{Prim} \mathcal{H})_{i}^{\tau}=\left|\mathcal{P} \cap \mathcal{H}_{i}\right|$ and $\bar{b}_{i}=\operatorname{dim}(\operatorname{Prim} \mathcal{H})_{i}^{-\tau}=\left|\overline{\mathcal{P}} \cap \mathcal{H}_{i}\right|$, then the multiplicities of $\beta_{\lambda, \bar{\lambda}}^{D}$ are $\left(\begin{array}{l}\boldsymbol{b} \\ \lambda\end{array}\right)\left(\begin{array}{l}\overline{\boldsymbol{b}} \\ \bar{\lambda}\end{array}\right)$. So it remains to show that the sequences $\boldsymbol{b}$ and $\overline{\boldsymbol{b}}$ are determined by the identities (8) and (9).

To see (8) (same argument as in the type A case): the PBW basis elements of degree $n$ are precisely the products over a multiset in $\mathcal{P}$ and a multiset in $\overline{\mathcal{P}}$, whose degrees total $n$.

To see (9): note that the PBW basis element $p_{1} \ldots p_{k} \bar{p}_{1} \ldots \bar{p}_{\bar{k}}$ is $\tau$-invariant if $\bar{k}$ is even, and $\tau$-negating if $\bar{k}$ is odd. Make a signed enumeration of such products, where each $\bar{p} \in \overline{\mathcal{P}}$ is signed, and all $p \in \mathcal{P}$ are unsigned, so that $p_{1} \ldots p_{k} \bar{p}_{1} \ldots \bar{p}_{\bar{k}}$ is $\tau$-negating if it is signed, and $\tau$-invariant if it is unsigned. Hence the coefficients are $\operatorname{dim} \mathcal{H}_{n}^{\tau}-\operatorname{dim} \mathcal{H}_{n}^{-\tau}$.

\section{The Hyperoctahedral-Riffle-Shuffle Operators}

This section focuses on the following four families of operators, whose rescaling (division by $a^{n}$ ) corresponds to $a$-handed hyperocatahedral riffle-shuffles of decks of $n$ cards, as studied in [BB92, Ful01, Ful02]. For a linear graded involution $\tau$ that is a Hopf ambimorphism, define

$$
\begin{aligned}
& \text { BRiffle } \tau_{a}^{+}:= \begin{cases}m \circ(\mathrm{id} \otimes \tau \otimes \mathrm{id} \otimes \tau \otimes \cdots \otimes \mathrm{id}) \circ \Delta^{[a]} & \text { if } a \text { is odd; } \\
m \circ(\mathrm{id} \otimes \tau \otimes \mathrm{id} \otimes \tau \otimes \cdots \otimes \tau) \circ \Delta^{[a]} & \text { if } a \text { is even; }\end{cases} \\
& \text { BRiffle } \tau_{a}^{-}:= \begin{cases}m \circ(\tau \otimes \mathrm{id} \otimes \tau \otimes \mathrm{id} \otimes \cdots \otimes \tau) \circ \Delta^{[a]} & \text { if } a \text { is odd; } \\
m \circ(\tau \otimes \mathrm{id} \otimes \tau \otimes \mathrm{id} \otimes \cdots \otimes \mathrm{id}) \circ \Delta^{[a]} & \text { if } a \text { is even. }\end{cases}
\end{aligned}
$$

In Sections 3.2 and 5 we will write $\tilde{\tau}$ in place of $\tau$ for an algebra antimorphism, to more clearly distinguish it from the case of an algebra morphism.

\subsection{Compositions and Properties}

A well-known feature of type A riffle-shuffles is that a $b$-handed shuffle followed by an $a$-handed shuffle has the same effect as an $a b$-handed shuffle; this allows repeated shuffles to be studied by considering a single many-handed shuffle. Below is the hyperoctahedral analogue, which is derived from Proposition 12; a special case appeared in [DFH13].

Proposition 24. Let $\mathcal{H}$ be a graded connected Hopf algebra, and $\tau: \mathcal{H} \rightarrow \mathcal{H}$ be a linear graded involution that is a Hopf ambimorphism. Under any one of these three conditions: 
i) $\mathcal{H}$ is commutative and $a$ is odd,

ii) $\mathcal{H}$ is cocommutative and $b$ is odd,

iii) $\mathcal{H}$ is commutative or cocommutative, and $\tau$ is not a Hopf morphism,

the operators in (11) compose as follows:

$$
\begin{aligned}
& \text { BRiffle } \tau_{a}^{+} \circ \text { BRiffle } \tau_{b}^{+}=\text {BRiffle } \tau_{a}^{-} \circ \text { BRiffle } \tau_{b}^{-}=\text {BRiffle } \tau_{a b}^{+} \\
& \text {BRiffle } \tau_{a}^{+} \circ \text { BRiffle } \tau_{b}^{-}=\text {BRiffle } \tau_{a}^{-} \circ \text { BRiffle } \tau_{b}^{+}=\text {BRiffle } \tau_{a b}^{-}
\end{aligned}
$$

Proof. View BRiffle $\tau_{a}^{+}$(resp. BRiffle $\tau_{a}^{-}$) as $\sum m \circ \Delta_{D(\tau)}$ over all $D$ with $a$ parts, where even parts (resp. odd parts) are negative. Then, on a commutative algebra $\mathcal{H}$, where $\tau$ is a Hopf morphism, Proposition 12.i gives BRiffle $\tau_{a}^{+} \circ$ BRiffle $\tau_{b}^{+}=$BRiffle $\tau_{a}^{-} \circ$ BRiffle $\tau_{b}^{-}=$ $\sum_{M} m \circ \Delta_{\text {wcomp }(M)}$, over all $b \times a$ matrices $M$ whose entries have the alternating sign pattern

$$
\begin{array}{llll}
+ & - & + & \ldots \\
- & + & - & \\
+ & - & \ddots
\end{array}
$$

If $a$ is odd, then the first row of this matrix ends with a positive entry, which is followed by a negative entry at the start of the second row when computing wcomp $(M)$. Similar considerations for other rows shows that $\operatorname{wcomp}(M)$ exactly runs through all signed weakcompositions with $b a$ parts whose even parts are negative. If instead $\tau$ is a coalgebra antimorphism, then the even rows must be read right to left when computing $\widetilde{w c o m p}_{D}(M)$. Thus, if $a$ is odd (resp. even), then the positive (resp. negative) entry at the end of the first row is followed by a negative (resp. positive) entry at the end of the second, so $\widetilde{w c o m p}_{D}(M)$ also runs through all signed weak-compositions with $b a$ parts whose even parts are negative.

The other cases are similar.

Next, we specialise Theorem 17 to obtain the spectrum of the hyperoctahedral-riffleshuffle operators:

Proposition 25. Let $\mathcal{H}$ be a graded connected Hopf algebra, and $\tau: \mathcal{H} \rightarrow \mathcal{H}$ be a linear graded involution that is a Hopf ambimorphism. Let $\boldsymbol{b}$ and $\overline{\boldsymbol{b}}$ be the sequences defined in (8) and (9). Consider the operators in (11) acting on $\mathcal{H}_{n}$. Write $[g] f$ to mean the cofficient of the monomial $g$ in the power series $f$. Then:

- for even a, the eigenvalues of BRiffle $\tau_{a}^{+}$and BRiffle $\tau_{a}^{-}$are $a^{l}$, with multiplicity $\left[x^{l} y^{n}\right] \prod_{i}\left(1-x y^{i}\right)^{-b_{i}} ;$ and 0 ;

- for odd a, the eigenvalues of BRiffle $\tau_{a}^{+}$are $a^{l}$, with multiplicity $\left[x^{l} y^{n}\right] \prod_{i}(1-$ $\left.x y^{i}\right)^{-b_{i}} \prod_{i}\left(1-y^{i}\right)^{-\bar{b}_{i}}$ 
- for odd a, the eigenvalues of BRiffle $\tau_{a}^{-}$are:

$$
\begin{aligned}
& -a^{l} \text {, with multiplicity }\left[x^{l} y^{n}\right] \prod_{i}\left(1-x y^{i}\right)^{-b_{i}} \frac{1}{2}\left(\prod_{i}\left(1+y^{i}\right)^{-\bar{b}_{i}}+\prod_{i}\left(1-y^{i}\right)^{-\bar{b}_{i}}\right) \\
& --a^{l} \text {, with multiplicity }\left[x^{l} y^{n}\right] \prod_{i}\left(1-x y^{i}\right)^{-b_{i}} \frac{1}{2}\left(\prod_{i}\left(1+y^{i}\right)^{-\bar{b}_{i}}-\prod_{i}\left(1-y^{i}\right)^{-\bar{b}_{i}}\right) .
\end{aligned}
$$

Proof. We generalise the ideas in Example 19. Let $\beta_{\lambda, \bar{\lambda}}^{a,+}$ (resp. $\beta_{\lambda, \bar{\lambda}}^{a,-}$ ) denote the eigenvalue of BRiffle $\tau_{a}^{+}$(resp. BRiffle $\tau_{a}^{-}$) corresponding to the double-partition $\lambda, \bar{\lambda}$; this is the signed count of set-compositions $B$ of $\{1,2, \ldots, l(\lambda)\} \cup\{\overline{1}, \overline{2}, \ldots, l(\bar{\lambda})\}$ into $a$ blocks, where the sign is the parity of signed integers in the even (resp. odd) blocks. First suppose $a$ is even. If $\bar{\lambda} \neq \varnothing$, then there is a sign-reversing involution on these set-compositions: if $\overline{1} \in B_{2 i-1}$ for some $i$, move it to $B_{2 i}$, and if $\overline{1} \in B_{2 i}$, move it to $B_{2 i-1}$. Hence $\beta_{\lambda, \bar{\lambda}}^{a,+}=$ $\beta_{\lambda, \bar{\lambda}}^{a,-}=0$ when $\bar{\lambda} \neq \varnothing$. If $\bar{\lambda}=\varnothing$, then all relevant set-compositions have positive sign, so $\beta_{\lambda, \bar{\lambda}}^{a,+}=\beta_{\lambda, \bar{\lambda}}^{a,-}=a^{l(\lambda)}$.

When $a$ is odd, the above signed involution is still defined when $\bar{\lambda} \neq \varnothing$ and $\overline{1} \notin B_{a}$. So, modify the signed involution to move the smallest $\bar{j}$ such that $\bar{j} \notin B_{a}$. This will be defined when $\bar{\lambda} \neq \varnothing$ and $\overline{1}, \overline{2}, \ldots, l(\bar{\lambda})$ are not all in $B_{a}$. Thus the only contributions to $\beta_{\lambda, \bar{\lambda}}^{a,+}$ or $\beta_{\lambda, \bar{\lambda}}^{a,-\bar{c}}$ come from set-compositions where $\overline{1}, \overline{2}, \ldots, l(\bar{\lambda})$ are all in $B_{a}$ - these are equivalent to the $a^{l(\lambda)}$ set-compositions of $\{1,2, \ldots, l(\lambda)\}$ into a blocks, and the sign is $(-1)^{l(\bar{\lambda})}$ for $\beta_{\lambda, \bar{\lambda}}^{a,-}$, and +1 always for $\beta_{\lambda, \bar{\lambda}}^{a,+}$.

To see the multiplicities: by Theorem 17.ii and Notation 16, the multiplicity of $\beta_{\lambda, \bar{\lambda}}^{a,+\bar{\lambda}}$ or $\beta_{\lambda, \bar{\lambda}}^{a,-}$ is

$$
\left(\begin{array}{l}
\boldsymbol{b} \\
\lambda
\end{array}\right)\left(\begin{array}{l}
\overline{\boldsymbol{b}} \\
\bar{\lambda}
\end{array}\right)=\left[x_{\lambda, \bar{\lambda}}\right] \prod_{i}\left(1-x_{i}\right)^{-b_{i}} \prod_{i}\left(1-\bar{x}_{i}\right)^{-\bar{b}_{i}}, \quad \text { where } x_{\lambda, \bar{\lambda}}:=x_{\lambda_{1}} \ldots x_{\lambda_{l(\lambda)}} \bar{x}_{\bar{\lambda}_{1}} \ldots \bar{x}_{\bar{\lambda}_{l(\bar{\lambda})}} .
$$

Make the substitution $x_{i} \mapsto x y^{i}, \bar{x}_{i} \mapsto y^{i}$ in the generating series on the right, so $x$ tracks the number of positive parts and $y$ tracks the total degree. Then $\left[x^{l} y^{n}\right] f(x, y)=$ $\sum\left[x_{\lambda, \bar{\lambda}}\right] f\left(x_{1}, x_{2}, \ldots, \bar{x}_{1}, \bar{x}_{2}, \ldots\right)$ summing over all double-partitions $\lambda, \bar{\lambda}$ of $n$ with $l$ parts - this handles the case of BRiffle $\tau_{a}^{+}$for odd $a$. For BRiffle $\tau_{a}^{-}$, the substitution $x_{i} \mapsto x y^{i}$, $\bar{x}_{i} \mapsto-y^{i}$ into Theorem 17.ii would introduce a sign when $\bar{\lambda}$ has an odd number of parts: $\left[x^{l} y^{n}\right] f(x, y)=\sum(-1)^{l(\bar{\lambda})}\left[x_{\lambda, \bar{\lambda}}\right] f\left(x_{1}, x_{2}, \ldots, \bar{x}_{1}, \bar{x}_{2}, \ldots\right)$. So, to sum only the coefficients of $x_{\lambda, \bar{\lambda}}$ when $\bar{\lambda}$ has an even number of parts, we can average the signed and unsigned substitutions, and similarly take their difference to isolate the coefficients of $x_{\lambda, \bar{\lambda}}$ for $\bar{\lambda}$ with an odd number of parts.

\section{$3.2 \quad$ Eigenvectors}

We give a basis of eigenvectors for the hyperoctahedral-riffle-shuffle operators. These will aid in computing the expectations of certain statistics under card-shuffling. The eigenvector formulas depend on whether the involution is an algebra morphism (Theorem 27 , written $\tau$ ) or antimorphism (Theorem 26, written $\tilde{\tau}$ ). The antimorphism case is easier, so we begin there. Let $\mathfrak{S}_{k}$ denote the symmetric group on $k$ objects. 
Theorem 26. Let $\mathcal{H}$ be a graded connected Hopf algebra, and $\tilde{\tau}: \mathcal{H} \rightarrow \mathcal{H}$ a linear graded involution that is an algebra antimorphism and a coalgebra morphism or antimorphism.

i) Take $p_{1}, \ldots, p_{k} \in(\operatorname{Prim\mathcal {H}})^{\tilde{\tau}}$ and $\bar{p}_{1}, \ldots, \bar{p}_{\bar{k}} \in(\text { Prim } \mathcal{H})^{-\tilde{\tau}}$ (where $k$ or $\bar{k}$ may be zero).

(a) If a is even, then

$$
\left(\sum_{\sigma \in \mathfrak{S}_{k}} p_{\sigma(1)} \ldots p_{\sigma(k)}\right) \bar{p}_{1} \ldots \bar{p}_{\bar{k}} \quad\left(\operatorname{resp} \cdot \bar{p}_{1} \ldots \bar{p}_{\bar{k}}\left(\sum_{\sigma \in \mathfrak{S}_{k}} p_{\sigma(1)} \ldots p_{\sigma(k)}\right)\right)
$$

is an eigenvector of BRiffle $\tilde{\tau}_{a}^{+}$(resp. BRiffle $\tilde{\tau}_{a}^{-}$), with eigenvalue a if $\bar{k}=0$, or eigenvalue 0 if $\bar{k}>0$.

(b) If a is odd, then

$$
\bar{p}_{1} \ldots \bar{p}_{\bar{k}}\left(\sum_{\sigma \in \mathfrak{S}_{k}} p_{\sigma(1)} \ldots p_{\sigma(k)}\right) \text { and }\left(\sum_{\sigma \in \mathfrak{S}_{k}} p_{\sigma(1)} \ldots p_{\sigma(k)}\right) \bar{p}_{1} \ldots \bar{p}_{\bar{k}}
$$

are eigenvectors of BRiffle $\tilde{\tau}_{a}^{+}$, with eigenvalue $a^{k}$.

(c) If a is odd, then

$$
\bar{p}_{1} \ldots \bar{p}_{\bar{k}}\left(\sum_{\sigma \in \mathfrak{S}_{k}} p_{\sigma(1)} \ldots p_{\sigma(k)}\right)+\left(\sum_{\sigma \in \mathfrak{S}_{k}} p_{\sigma(1)} \ldots p_{\sigma(k)}\right) \bar{p}_{1} \ldots \bar{p}_{\bar{k}}
$$

is an eigenvector of BRiffle $\tilde{\tau}_{a}^{-}$, for odd a, with eigenvalue $(-1)^{\bar{k}} a^{k}$.

ii) If $\mathcal{P}, \overline{\mathcal{P}}$ are ordered bases of $(\operatorname{Prim\mathcal {H}})^{\tilde{\tau}},(\text { Prim } \mathcal{H})^{-\tilde{\tau}}$ respectively, then the vectors of any fixed format in $i$ above, over all choices of $p_{1} \preceq \cdots \preceq p_{k} \in \mathcal{P}$ and $\bar{p}_{1} \preceq \cdots \preceq$ $\bar{p}_{\bar{k}} \in \overline{\mathcal{P}}$ (allowing $k$ and $\bar{k}$ to vary), are linearly independent.

iii) Furthermore, if $\mathcal{H}$ is cocommutative, then the sets described in ii above are bases of eigenvectors for the appropriate BRiffle $\tilde{\tau}$ operator.

Theorem 27. Let $\mathcal{H}$ be a graded connected Hopf algebra, and $\tau: \mathcal{H} \rightarrow \mathcal{H}$ a linear graded involution that is an algebra morphism and a coalgebra morphism or antimorphism. Fix a odd.

i) For $p_{1}, \ldots, p_{k} \in(\operatorname{Prim} \mathcal{H})^{\tau}$ and $\bar{p}_{1}, \ldots, \bar{p}_{\bar{k}} \in(\operatorname{Prim\mathcal {H}})^{-\tau}$ (where $k$ or $\bar{k}$ may be zero),

$$
\sum_{B} \coprod_{i \in B_{1}} \bar{p}_{i}\left(\sum_{\sigma \in \mathfrak{S}_{k}} p_{\sigma(1)} \ldots p_{\sigma(k)}\right) \prod_{i \in B_{2}} \bar{p}_{i}
$$

summing over all set-compositions $B=B_{1} \mid B_{2}$ of $\{1,2, \ldots, \bar{k}\}$ into 2 blocks, is an eigenvector of BRiffle $\tau_{a}^{+}$with eigenvalue $a^{k}$, and an eigenvector of BRiffle $\tau_{a}^{-}$with eigenvalue $(-1)^{\bar{k}} a^{k}$. (โ denotes that the product should be taken in the reverse order, with large index $i$ on the left and small index $i$ on the right - see Example 28 below.) 
ii) If $\mathcal{P}, \overline{\mathcal{P}}$ are ordered bases of $(\text { Prim } \mathcal{H})^{\tau},(\text { Prim } \mathcal{H})^{-\tau}$ respectively, then the vectors in (12), over all choices of $p_{1} \preceq \cdots \preceq p_{k} \in \mathcal{P}$ and $\bar{p}_{1} \preceq \cdots \preceq \bar{p}_{\bar{k}} \in \overline{\mathcal{P}}$ (allowing $k$ and $\bar{k}$ to vary), are linearly independent.

iii) Furthermore, if $\mathcal{H}$ is cocommutative, then the set described in ii above is a basis of eigenvectors for BRiffle $\tau_{a}^{+}$and BRiffle $\tau_{a}^{-}$.

Example 28. If $k=2$ and $\bar{k}=3$, then the eigenvector given by (12) is

$$
\begin{aligned}
& \bar{p}_{3} \bar{p}_{2} \bar{p}_{1}\left(p_{1} p_{2}+p_{2} p_{1}\right)+\bar{p}_{2} \bar{p}_{1}\left(p_{1} p_{2}+p_{2} p_{1}\right) \bar{p}_{3} \\
+ & \bar{p}_{3} \bar{p}_{1}\left(p_{1} p_{2}+p_{2} p_{1}\right) \bar{p}_{2}+\bar{p}_{3} \bar{p}_{2}\left(p_{1} p_{2}+p_{2} p_{1}\right) \bar{p}_{1} \\
+ & \bar{p}_{1}\left(p_{1} p_{2}+p_{2} p_{1}\right) \bar{p}_{2} \bar{p}_{3}+\bar{p}_{2}\left(p_{1} p_{2}+p_{2} p_{1}\right) \bar{p}_{1} \bar{p}_{3} \\
+ & \bar{p}_{3}\left(p_{1} p_{2}+p_{2} p_{1}\right) \bar{p}_{1} \bar{p}_{2}+\left(p_{1} p_{2}+p_{2} p_{1}\right) \bar{p}_{1} \bar{p}_{2} \bar{p}_{3} .
\end{aligned}
$$

For a further example in the free associative algebra, see Example 43.

When $a$ is even, we can only identify the eigenvectors of BRiffle $\tau_{a}^{+}$and BRiffle $\tau_{a}^{-}$with non-zero eigenvalues:

Theorem 29. Let $\mathcal{H}$ be a graded connected Hopf algebra, and $\tau: \mathcal{H} \rightarrow \mathcal{H}$ a linear graded involution that is an algebra morphism and a coalgebra morphism or antimorphism. Fix a even.

i) For $p_{1}, \ldots, p_{k} \in(\operatorname{Prim} \mathcal{H})^{\tau}$,

$$
\sum_{\sigma \in \mathfrak{S}_{k}} p_{\sigma(1)} \ldots p_{\sigma(k)}
$$

is an eigenvector of $\mathrm{BRiffle} \tau_{a}^{+}$and $\mathrm{BRiffle} \tau_{a}^{-}$with eigenvalue $a^{k}$.

ii) If $\mathcal{P}$ is an ordered basis of $(\text { Prim } \mathcal{H})^{\tau}$, then the vectors described in $i$ above, over all choices of $p_{1} \preceq \cdots \preceq p_{k} \in \mathcal{P}$, are linearly independent.

iii) Furthermore, if $\mathcal{H}$ is cocommutative, then the set of eigenvectors described in ii above, for each fixed $k$, is a basis for the eigenspace of eigenvalue $a^{k}$, for both BRiffle $\tau_{a}^{+}$and BRiffle $\tau_{a}^{-}$, and there are no generalised eigenvectors for these eigenvalues. In other words, the eigenvectors described in ii, over all values of $k$, is a basis for a complement of the generalised eigenspace of eigenvalue 0 .

The proof of Theorems 26 and 27 follow the same structure of an induction on $\bar{k}$, the number of $\tau$-negating (or $\tilde{\tau}$-negating) primitives; Lemma 30 below is the common base case $(\bar{k}=0)$, and to increase $\bar{k}$ we use a sign-reversing involution argument: Lemma 32 for an algebra morphism, and Lemma 31 for an algebra antimorphism. Since Theorem 29 does not involve $\tau$-negating primitives, its proof requires only the previous "base case" of Lemma 30, plus a counting argument. Note that this Lemma applies to all four types of Hopf ambimorphism, i.e. to both $\tau$ and $\tilde{\tau}$. 
Lemma 30. Let $\mathcal{H}$ be a graded connected Hopf algebra, and $\tau: \mathcal{H} \rightarrow \mathcal{H}$ be a linear graded involution that is a Hopf ambimorphism. For $p_{1}, \ldots, p_{k} \in(\text { Prim } \mathcal{H})^{\tau}$, the symmetrised product $\sum_{\sigma \in \mathfrak{S}_{k}} p_{\sigma(1)} \ldots p_{\sigma(k)}$ is an eigenvector of BRiffle $\tau_{a}^{+}$and BRiffle $\tau_{a}^{-}$, of eigenvalue $a^{k}$.

Proof. The argument is essentially the same for all four operators of (11). For concreteness, consider first BRiffle $\tilde{\tau}_{a}^{+}$where $a$ is odd.

$$
\Delta^{[a]}\left(p_{1} \ldots p_{k}\right)=\sum_{B}\left(\prod_{i \in B_{1}} p_{i}\right) \otimes\left(\prod_{i \in B_{2}} p_{i}\right) \otimes \cdots \otimes\left(\prod_{i \in B_{a}} p_{i}\right),
$$

summing over all set-compositions $B$ of $\{1, \ldots, k\}$ with $a$ blocks. So

$$
(\mathrm{id} \otimes \tilde{\tau} \otimes \mathrm{id} \otimes \tilde{\tau} \cdots \otimes \mathrm{id}) \Delta^{[a]}\left(p_{1} \ldots p_{k}\right)=\sum_{B}\left(\prod_{i \in B_{1}} p_{i}\right) \otimes\left(\prod_{i \in B_{2}} p_{i}\right) \otimes \cdots \otimes\left(\prod_{i \in B_{a}} p_{i}\right)
$$

where the arrows above the product signs indicate reversing the order of the product. Hence BRiffle $\tilde{\tau}_{a}^{+}\left(p_{1} \ldots p_{k}\right)$ is a sum of products of the same primitives, in a different order, and the permissable orders do not depend on anything particular about each $p_{i}$. Thus $\sum_{\sigma \in \mathfrak{S}_{k}} p_{\sigma(1)} \ldots p_{\sigma(k)}$ is an eigenvector of BRiffle $\tilde{\tau}_{a}^{+}$and the eigenvalue is the number of summands in (13), i.e. the number of set-compositions of $\{1, \ldots, k\}$ with $a$ blocks. Since each number can belong to any block, the required number is $a^{k}$.

The other seven cases (BRiffle $\tau_{a}^{+}$with $a$ even, BRiffle $\tau_{a}^{-}$with $a$ even or $a$ odd, BRiffle $\tilde{\tau}_{a}^{+}$and BRiffle $\tilde{\tau}_{a}^{-}$for all values of $a$ ) are similar: the directions of the products in (13) are different, but each summand is nevertheless a product of the same primitives in a different order, hence the argument above applies.

Proof of Theorem 29. Let $\beta_{\lambda, \bar{\lambda}}^{a,+}$ (resp. $\beta_{\lambda, \bar{\lambda}}^{a,-}$ ) denote the eigenvalue of BRiffle $\tau_{a}^{+}$(resp. BRiffle $\tau_{a}^{-}$) corresponding to the double-partition $\lambda, \bar{\lambda}$, as in the proof of Proposition 25.

i) Immediate from Lemma 30 .

ii) Work in the subalgebra of $\mathcal{H}$ that is the universal enveloping algebra of $(\operatorname{Prim\mathcal {H}})^{\tau}$, with a PBW basis formed from $\mathcal{P}$. As explained in the Triangularity Lemma (23), the eigenvector $\sum_{\sigma \in \mathfrak{S}_{k}} p_{\sigma(1)} \ldots p_{\sigma(k)}$ is a sum of products of $p_{1}, \ldots, p_{k}$ in different orders, and hence its highest length term in this PBW basis is $k ! p_{1} \ldots p_{k}$. Thus each eigenvector has a different highest length term, and so they are linearly independent.

iii) Let $\overline{\mathcal{P}}$ be a basis of $(\operatorname{Prim} \mathcal{H})^{-\tau}$. As noted in the paragraph after Lemma 23: in a cocommutative Hopf algebra, the multiplicity of the eigenvalue $\beta_{\lambda, \bar{\lambda}}^{a,+}$ or $\beta_{\lambda, \bar{\lambda}}^{a,-}$ is the number of multiset pairs $\left\{p_{1}, \ldots, p_{k}\right\} \subseteq \mathcal{P},\left\{\bar{p}_{1} \ldots, \bar{p}_{\bar{k}}\right\} \subseteq \overline{\mathcal{P}}$ where $\lambda=$ $\left(\operatorname{deg} p_{1}, \ldots, \operatorname{deg} p_{k}\right)$ and $\bar{\lambda}=\left(\operatorname{deg} \bar{p}_{1}, \ldots, \operatorname{deg} \bar{p}_{\bar{k}}\right)$. The constructed eigenvectors accounts for all cases where $\bar{\lambda}=\varnothing$. And, in the proof of Proposition 24, a signreversing involution showed that $\beta_{\lambda, \bar{\lambda}}^{a,+}=\beta_{\lambda, \bar{\lambda}}^{a,-}=0$ if $\bar{\lambda} \neq \varnothing$. 
The following two lemmas are the sign-reversing involutions required to inductively construct eigenvectors.

Lemma 31. Let $\mathcal{H}$ be a graded connected Hopf algebra, and $\tilde{\tau}: \mathcal{H} \rightarrow \mathcal{H}$ be a linear graded involution that is an algebra antimorphism and a coalgebra morphism or antimorphism. Let $\bar{p}$ be a $\tilde{\tau}$-negating primitive element of $\mathcal{H}$, and $s$ be any product of primitive elements. Then

for $a$ odd : $\quad$ BRiffle $\tilde{\tau}_{a}^{+}(\bar{p} s)=\bar{p} \operatorname{BRiffle} \tilde{\tau}_{a}^{+}(s), \quad$ BRiffle $\tilde{\tau}_{a}^{+}(s \bar{p})=\operatorname{BRiffle} \tilde{\tau}_{a}^{+}(s) \bar{p}$, BRiffle $\tilde{\tau}_{a}^{-}(\bar{p} s)=-$ BRiffle $\tilde{\tau}_{a}^{-}(s) \bar{p}, \quad$ BRiffle $\tilde{\tau}_{a}^{-}(s \bar{p})=-\bar{p}$ BRiffle $\tilde{\tau}_{a}^{-}(s)$;

for a even: BRiffle $\tilde{\tau}_{a}^{+}(s \bar{p})=0$,

$$
\text { BRiffle } \tilde{\tau}_{a}^{-}(\bar{p} s)=0
$$

Proof. Consider first the equations on the left, involving $\bar{p} s$. Let $s=p_{1} \ldots p_{k}$ and, for clearer exposition, first set $a=5$.

$$
\begin{aligned}
\Delta^{[5]}\left(\bar{p} p_{1} \ldots p_{k}\right)=\sum_{B}(\bar{p} & \left.\prod_{i \in B_{1}} p_{i}\right) \otimes\left(\prod_{i \in B_{2}} p_{i}\right) \otimes\left(\prod_{i \in B_{3}} p_{i}\right) \otimes\left(\prod_{i \in B_{4}} p_{i}\right) \otimes\left(\prod_{i \in B_{5}} p_{i}\right) \\
& +\left(\prod_{i \in B_{1}} p_{i}\right) \otimes\left(\bar{p} \prod_{i \in B_{2}} p_{i}\right) \otimes\left(\prod_{i \in B_{3}} p_{i}\right) \otimes\left(\prod_{i \in B_{4}} p_{i}\right) \otimes\left(\prod_{i \in B_{5}} p_{i}\right) \\
& +\left(\prod_{i \in B_{1}} p_{i}\right) \otimes\left(\prod_{i \in B_{2}} p_{i}\right) \otimes\left(\bar{p} \prod_{i \in B_{3}} p_{i}\right) \otimes\left(\prod_{i \in B_{4}} p_{i}\right) \otimes\left(\prod_{i \in B_{5}} p_{i}\right) \\
& +\left(\prod_{i \in B_{1}} p_{i}\right) \otimes\left(\prod_{i \in B_{2}} p_{i}\right) \otimes\left(\prod_{i \in B_{3}} p_{i}\right) \otimes\left(\bar{p} \prod_{i \in B_{4}} p_{i}\right) \otimes\left(\prod_{i \in B_{5}} p_{i}\right) \\
& +\left(\prod_{i \in B_{1}} p_{i}\right) \otimes\left(\prod_{i \in B_{2}} p_{i}\right) \otimes\left(\prod_{i \in B_{3}} p_{i}\right) \otimes\left(\prod_{i \in B_{4}} p_{i}\right) \otimes\left(\bar{p} \prod_{i \in B_{5}} p_{i}\right)
\end{aligned}
$$

summing over all set-compositions $B$ of $\{1,2, \ldots, k\}$ into 5 parts. (Each such setcomposition contributed 5 terms, for all 5 possible blocks where $\bar{p}$ may be assigned.) 
So

$$
\begin{aligned}
\text { BRiffle } \tilde{\tau}_{5}^{+}\left(\bar{p} p_{1} \ldots p_{k}\right)=\sum_{B} & \left(\bar{p} \prod_{i \in B_{1}} p_{i}\right) \tilde{\tau}\left(\prod_{i \in B_{2}} p_{i}\right)\left(\prod_{i \in B_{3}} p_{i}\right) \tilde{\tau}\left(\prod_{i \in B_{4}} p_{i}\right)\left(\prod_{i \in B_{5}} p_{i}\right) \\
& -\left(\prod_{i \in B_{1}} p_{i}\right)\left[\tilde{\tau}\left(\prod_{i \in B_{2}} p_{i}\right) \bar{p}\right]\left(\prod_{i \in B_{3}} p_{i}\right) \tilde{\tau}\left(\prod_{i \in B_{4}} p_{i}\right)\left(\prod_{i \in B_{5}} p_{i}\right) \\
& +\left(\prod_{i \in B_{1}} p_{i}\right) \tilde{\tau}\left(\prod_{i \in B_{2}} p_{i}\right)\left(\bar{p} \prod_{i \in B_{3}} p_{i}\right) \tilde{\tau}\left(\prod_{i \in B_{4}} p_{i}\right)\left(\prod_{i \in B_{5}} p_{i}\right) \\
& -\left(\prod_{i \in B_{1}} p_{i}\right) \tilde{\tau}\left(\prod_{i \in B_{2}} p_{i}\right)\left(\prod_{i \in B_{3}} p_{i}\right)\left[\tilde{\tau}\left(\prod_{i \in B_{4}} p_{i}\right) \bar{p}\right]\left(\prod_{i \in B_{5}} p_{i}\right) \\
& +\left(\prod_{i \in B_{1}} p_{i}\right) \tilde{\tau}\left(\prod_{i \in B_{2}} p_{i}\right)\left(\prod_{i \in B_{3}} p_{i}\right) \tilde{\tau}\left(\prod_{i \in B_{4}} p_{i}\right)\left(\bar{p} \prod_{i \in B_{5}} p_{i}\right) .
\end{aligned}
$$

(The second factor of the second line and the fourth factor of the fourth line uses, for all $x$, that $\tilde{\tau}(\bar{p} x)=\tilde{\tau}(x) \tilde{\tau}(\bar{p})=-\tilde{\tau}(x) \bar{p}$.) Note that, for each fixed set-composition $B$, the second and third lines of (16) are equal, except for their opposite sign, so they cancel. Similarly, the fourth and fifth lines cancel. Thus only the first line remains, and that is precisely $\bar{p}$ BRiffle $\tilde{\tau}_{5}^{+}\left(p_{1} \ldots p_{k}\right)$.

By this argument, whenever $a$ is odd, BRiffle $\tilde{\tau}_{a}^{+}\left(\bar{p} p_{1} \ldots p_{k}\right)$ is a sum of $a$ terms for each set-composition of $\{1,2, \ldots, k\}$ into $a$ parts, and each even term cancels with the following odd term, so only the first term remains and these give $\bar{p} \operatorname{BRiffle} \tilde{\tau}_{a}^{+}\left(p_{1} \ldots p_{k}\right)$.

Similarly,

$$
\begin{aligned}
\text { BRiffle } \tilde{\tau}_{a}^{-}\left(\bar{p} p_{1} \ldots p_{k}\right)=\sum_{B}- & {\left[\tilde{\tau}\left(\prod_{i \in B_{1}} p_{i}\right) \bar{p}\right]\left(\prod_{i \in B_{2}} p_{i}\right) \tilde{\tau}\left(\prod_{i \in B_{3}} p_{i}\right) \ldots } \\
& +\tilde{\tau}\left(\prod_{i \in B_{1}} p_{i}\right)\left(\bar{p} \prod_{i \in B_{2}} p_{i}\right) \tilde{\tau}\left(\prod_{i \in B_{3}} p_{i}\right) \ldots \\
& -\tilde{\tau}\left(\prod_{i \in B_{1}} p_{i}\right)\left(\prod_{i \in B_{2}} p_{i}\right)\left[\tilde{\tau}\left(\prod_{i \in B_{3}} p_{i}\right) \bar{p}\right] \ldots \\
& +\ldots,
\end{aligned}
$$

so each odd line cancels with the line below. Hence, if $a$ is even, the sum entirely cancels and is thus 0 ; if $a$ is odd, the last line remains:

$$
\begin{aligned}
\operatorname{BRiffle} \tilde{\tau}_{a}^{-}\left(\bar{p} p_{1} \ldots p_{k}\right) & =\sum_{B}-\tilde{\tau}\left(\prod_{i \in B_{1}} p_{i}\right)\left(\prod_{i \in B_{2}} p_{i}\right) \tilde{\tau}\left(\prod_{i \in B_{3}} p_{i}\right) \ldots\left[\tilde{\tau}\left(\prod_{i \in B_{a}} p_{i}\right) \bar{p}\right] \\
& =\operatorname{BRiffle} \tilde{\tau}_{a}^{-}\left(p_{1} \ldots p_{k}\right) \bar{p} .
\end{aligned}
$$


For $s \bar{p}$, the calculations are very similar. Again, let $s=p_{1} \ldots p_{k}$.

$$
\begin{aligned}
\text { BRiffle } \tilde{\tau}_{a}^{+}\left(p_{1} \ldots p_{k} \bar{p}\right)=\sum_{B} & {\left[\left(\prod_{i \in B_{1}} p_{i}\right) \bar{p}\right] \tilde{\tau}\left(\prod_{i \in B_{2}} p_{i}\right)\left(\prod_{i \in B_{3}} p_{i}\right) \ldots } \\
& -\left(\prod_{i \in B_{1}} p_{i}\right)\left[\bar{p} \tilde{\tau}\left(\prod_{i \in B_{2}} p_{i}\right)\right]\left(\prod_{i \in B_{3}} p_{i}\right) \ldots \\
& +\left(\prod_{i \in B_{1}} p_{i}\right) \tilde{\tau}\left(\prod_{i \in B_{2}} p_{i}\right)\left[\left(\prod_{i \in B_{3}} p_{i}\right) \bar{p}\right] \ldots \\
& -\ldots,
\end{aligned}
$$

so each odd line cancels with the line below, leaving the last line (i.e. BRiffle $\left.\tilde{\tau}_{a}^{+}\left(p_{1} \ldots p_{k}\right) \bar{p}\right)$ if $a$ is odd, and cancelling entirely if $a$ is even. And, for $a$ odd,

$$
\begin{aligned}
\text { BRiffle } \tilde{\tau}_{a}^{-}\left(p_{1} \ldots p_{k} \bar{p}\right)=\sum_{B}- & {\left[\bar{p} \tilde{\tau}\left(\prod_{i \in B_{1}} p_{i}\right)\right]\left(\prod_{i \in B_{2}} p_{i}\right) \tilde{\tau}\left(\prod_{i \in B_{3}} p_{i}\right) \ldots \tilde{\tau}\left(\prod_{i \in B_{a}} p_{i}\right) } \\
& +\tilde{\tau}\left(\prod_{i \in B_{1}} p_{i}\right)\left[\left(\prod_{i \in B_{2}} p_{i}\right) \bar{p}\right] \tilde{\tau}\left(\prod_{i \in B_{3}} p_{i}\right) \ldots\left(\prod_{i \in B_{a}} p_{i}\right) \\
& -\tilde{\tau}\left(\prod_{i \in B_{1}} p_{i}\right)\left(\prod_{i \in B_{2}} p_{i}\right)\left[\bar{p} \tilde{\tau}\left(\prod_{i \in B_{3}} p_{i}\right)\right] \ldots \tilde{\tau}\left(\prod_{i \in B_{a}} p_{i}\right) \\
& +\ldots \\
& -\tilde{\tau}\left(\prod_{i \in B_{1}} p_{i}\right)\left(\prod_{i \in B_{2}} p_{i}\right) \tilde{\tau}\left(\prod_{i \in B_{3}} p_{i}\right) \ldots\left[\bar{p} \tilde{\tau}\left(\prod_{i \in B_{a}} p_{i}\right)\right] .
\end{aligned}
$$

where each even line cancels with the line below, leaving the first line, which is exactly $\bar{p}$ BRiffle $\tilde{\tau}_{a}^{-}\left(p_{1} \ldots p_{k}\right)$.

Lemma 32. Let $\mathcal{H}$ be a graded connected Hopf algebra, and $\tau: \mathcal{H} \rightarrow \mathcal{H}$ be a linear graded involution that is an algebra morphism and a coalgebra morphism or antimorphism. Let $\bar{p}$ be a $\tau$-negating primitive element of $\mathcal{H}$, and $s$ be any product of primitive elements.

Then, if a is odd:

$$
\begin{aligned}
& \text { BRiffle } \tau_{a}^{+}(\bar{p} s+s \bar{p})=\bar{p} \text { BRiffle } \tau_{a}^{+}(s)+\text { BRiffle } \tau_{a}^{+}(s) \bar{p} \\
& \text { BRiffle } \tau_{a}^{-}(\bar{p} s+s \bar{p})=-\bar{p} \operatorname{BRiffle} \tau_{a}^{-}(s)-\text { BRiffle } \tau_{a}^{-}(s) \bar{p}
\end{aligned}
$$

Proof. Let $s=p_{1} \ldots p_{k}$, and, as in the proof of Lemma 31, first set $a=5$ for clearer 
exposition. From (15), we have

$$
\begin{aligned}
\text { BRiffle } \tau_{a}^{+}\left(\bar{p} p_{1} \ldots p_{k}\right)=\sum_{B} & \left(\bar{p} \prod_{i \in B_{1}} p_{i}\right) \tau\left(\prod_{i \in B_{2}} p_{i}\right)\left(\prod_{i \in B_{3}} p_{i}\right) \tau\left(\prod_{i \in B_{4}} p_{i}\right)\left(\prod_{i \in B_{5}} p_{i}\right) \\
& -\left(\prod_{i \in B_{1}} p_{i}\right)\left[\bar{p} \tau\left(\prod_{i \in B_{2}} p_{i}\right)\right]\left(\prod_{i \in B_{3}} p_{i}\right) \tau\left(\prod_{i \in B_{4}} p_{i}\right)\left(\prod_{i \in B_{5}} p_{i}\right) \\
& +\left(\prod_{i \in B_{1}} p_{i}\right) \tau\left(\prod_{i \in B_{2}} p_{i}\right)\left(\bar{p} \prod_{i \in B_{3}} p_{i}\right) \tau\left(\prod_{i \in B_{4}} p_{i}\right)\left(\prod_{i \in B_{5}} p_{i}\right) \\
& -\left(\prod_{i \in B_{1}} p_{i}\right) \tau\left(\prod_{i \in B_{2}} p_{i}\right)\left(\prod_{i \in B_{3}} p_{i}\right)\left[\bar{p} \tau\left(\prod_{i \in B_{4}} p_{i}\right)\right]\left(\prod_{i \in B_{5}} p_{i}\right) \\
& +\left(\prod_{i \in B_{1}} p_{i}\right) \tau\left(\prod_{i \in B_{2}} p_{i}\right)\left(\prod_{i \in B_{3}} p_{i}\right) \tau\left(\prod_{i \in B_{4}} p_{i}\right)\left(\bar{p} \prod_{i \in B_{5}} p_{i}\right)
\end{aligned}
$$

and similarly

$$
\begin{aligned}
\text { BRiffle } \tau_{a}^{+}\left(p_{1} \ldots p_{k} \bar{p}\right)=\sum_{B} & {\left[\left(\prod_{i \in B_{1}} p_{i}\right) \bar{p}\right] \tau\left(\prod_{i \in B_{2}} p_{i}\right)\left(\prod_{i \in B_{3}} p_{i}\right) \tau\left(\prod_{i \in B_{4}} p_{i}\right)\left(\prod_{i \in B_{5}} p_{i}\right) } \\
& -\left(\prod_{i \in B_{1}} p_{i}\right)\left[\tau\left(\prod_{i \in B_{2}} p_{i}\right) \bar{p}\right]\left(\prod_{i \in B_{3}} p_{i}\right) \tau\left(\prod_{i \in B_{4}} p_{i}\right)\left(\prod_{i \in B_{5}} p_{i}\right) \\
& +\left(\prod_{i \in B_{1}} p_{i}\right) \tau\left(\prod_{i \in B_{2}} p_{i}\right)\left[\left(\prod_{i \in B_{3}} p_{i}\right) \bar{p}\right] \tau\left(\prod_{i \in B_{4}} p_{i}\right)\left(\prod_{i \in B_{5}} p_{i}\right) \\
& -\left(\prod_{i \in B_{1}} p_{i}\right) \tau\left(\prod_{i \in B_{2}} p_{i}\right)\left(\prod_{i \in B_{3}} p_{i}\right)\left[\tau\left(\prod_{i \in B_{4}} p_{i}\right) \bar{p}\right]\left(\prod_{i \in B_{5}} p_{i}\right) \\
& +\left(\prod_{i \in B_{1}} p_{i}\right) \tau\left(\prod_{i \in B_{2}} p_{i}\right)\left(\prod_{i \in B_{3}} p_{i}\right) \tau\left(\prod_{i \in B_{4}} p_{i}\right)\left[\left(\prod_{i \in B_{5}} p_{i}\right) \bar{p}\right] .
\end{aligned}
$$

Note that, for each fixed set-composition $B$ of $\{1,2, \ldots, k\}$ into 5 parts, the second line of (18) is equal to the first line of (19) except for the opposite sign. So these will cancel in BRiffle $\tau_{a}^{+}\left(\bar{p} p_{1} \ldots p_{k}\right)+$ BRiffle $\tau_{a}^{+}\left(p_{1} \ldots p_{k} \bar{p}\right)$, and similarly the third line of (18) and the second line of (19) will cancel, and the fourth line of (18) and the third line of (19) will cancel, and so on. Hence BRiffle $\tau_{a}^{+}\left(\bar{p} p_{1} \ldots p_{k}\right)+\operatorname{BRiffle} \tau_{a}^{+}\left(p_{1} \ldots p_{k} \bar{p}\right)$ is equal to the first line of (18) plus the last line of (19) - these are equal respectively to $\bar{p}$ BRiffle $\tau_{a}^{+}\left(p_{1} \ldots p_{k}\right)$ and BRiffle $\tau_{a}^{+}\left(p_{1} \ldots p_{k}\right) \bar{p}$. The same cancellations occur for other odd values of $a$. 
As for BRiffle $\tau_{a}^{-}$(for $a$ odd):

$$
\begin{aligned}
\text { BRiffle } \tau_{a}^{-}\left(\bar{p} p_{1} \ldots p_{k}\right)=\sum_{B}- & {\left[\bar{p} \tau\left(\prod_{i \in B_{1}} p_{i}\right)\right]\left(\prod_{i \in B_{2}} p_{i}\right) \tau\left(\prod_{i \in B_{3}} p_{i}\right) \ldots \tau\left(\prod_{i \in B_{a}} p_{i}\right) } \\
& +\tau\left(\prod_{i \in B_{1}} p_{i}\right)\left(\bar{p} \prod_{i \in B_{2}} p_{i}\right) \tau\left(\prod_{i \in B_{3}} p_{i}\right) \ldots \tau\left(\prod_{i \in B_{a}} p_{i}\right) \\
& -\tau\left(\prod_{i \in B_{1}} p_{i}\right)\left(\prod_{i \in B_{2}} p_{i}\right)\left[\bar{p} \tau\left(\prod_{i \in B_{3}} p_{i}\right)\right] \ldots \tau\left(\prod_{i \in B_{a}} p_{i}\right) \\
& +\ldots \\
& -\tau\left(\prod_{i \in B_{1}} p_{i}\right)\left(\prod_{i \in B_{2}} p_{i}\right) \tau\left(\prod_{i \in B_{3}} p_{i}\right) \ldots\left[\bar{p} \tau\left(\prod_{i \in B_{a}} p_{i}\right)\right]
\end{aligned}
$$

and

$$
\begin{aligned}
\text { BRiffle } \tau_{a}^{-}\left(p_{1} \ldots p_{k} \bar{p}\right)=\sum_{B}- & {\left[\tau\left(\prod_{i \in B_{1}} p_{i}\right) \bar{p}\right]\left(\prod_{i \in B_{2}} p_{i}\right) \tau\left(\prod_{i \in B_{3}} p_{i}\right) \ldots \tau\left(\prod_{i \in B_{a}} p_{i}\right) } \\
& +\tau\left(\prod_{i \in B_{1}} p_{i}\right)\left[\left(\prod_{i \in B_{2}} p_{i}\right) \bar{p}\right] \tau\left(\prod_{i \in B_{3}} p_{i}\right) \ldots \tau\left(\prod_{i \in B_{a}} p_{i}\right) \\
& -\tau\left(\prod_{i \in B_{1}} p_{i}\right)\left(\prod_{i \in B_{2}} p_{i}\right)\left[\tau\left(\prod_{i \in B_{3}} p_{i}\right) \bar{p}\right] \ldots \tau\left(\prod_{i \in B_{a}} p_{i}\right) \\
& +\ldots \\
& -\tau\left(\prod_{i \in B_{1}} p_{i}\right)\left(\prod_{i \in B_{2}} p_{i}\right) \tau\left(\prod_{i \in B_{3}} p_{i}\right) \ldots\left[\tau\left(\prod_{i \in B_{a}} p_{i}\right) \bar{p}\right] .
\end{aligned}
$$

So, when (20) and (21) are summed, again the $i$ th line of (20) cancels with the $i+1$ th line of (21), leaving the first line of (20) and the last line of (21).

With these sign-reversing involutions in place, we proceed to prove the eigenvector formulas.

Proof of Theorem 26. i) First note that all lines in (14) are linear in $s$, i.e. Lemma 31 holds when $s$ is a linear combination of products of primitives.

(a) The $\bar{k}=0$ case is Lemma 30. For $\bar{k}>0$, use the 3rd and 4th lines of (14), with $s=\left(\sum_{\sigma \in \mathfrak{S}_{k}} p_{\sigma(1)} \ldots p_{\sigma(k)}\right) \bar{p}_{1} \ldots \bar{p}_{\bar{k}-1}$ and $s=\bar{p}_{2} \ldots \bar{p}_{\bar{k}}\left(\sum_{\sigma \in \mathfrak{S}_{k}} p_{\sigma(1)} \ldots p_{\sigma(k)}\right)$ respectively. 
(b) Proceed by induction on $\bar{k}$, the base case $\bar{k}=0$ being Lemma 30. By the first line of (14):

$$
\begin{aligned}
& \text { BRiffle } \tilde{\tau}_{a}^{+}\left(\bar{p}_{1} \ldots \bar{p}_{\bar{k}} \sum_{\sigma \in \mathfrak{S}_{k}} p_{\sigma(1)} \ldots p_{\sigma(k)}\right) \\
= & \bar{p}_{1} \text { BRiffle } \tilde{\tau}_{a}^{+}\left(\bar{p}_{2} \ldots \bar{p}_{\bar{k}} \sum_{\sigma \in \mathfrak{S}_{k}} p_{\sigma(1)} \ldots p_{\sigma(k)}\right) \\
= & \bar{p}_{1} a^{k} \bar{p}_{2} \ldots \bar{p}_{\bar{k}} \sum_{\sigma \in \mathfrak{S}_{k}} p_{\sigma(1)} \ldots p_{\sigma(k)} ;
\end{aligned}
$$

and

$$
\begin{aligned}
& \text { BRiffle } \tilde{\tau}_{a}^{+}\left[\left(\sum_{\sigma \in \mathfrak{S}_{k}} p_{\sigma(1)} \ldots p_{\sigma(k)}\right) \bar{p}_{1} \ldots \bar{p}_{\bar{k}}\right] \\
= & \operatorname{BRiffle} \tilde{\tau}_{a}^{+}\left[\left(\sum_{\sigma \in \mathfrak{S}_{k}} p_{\sigma(1)} \ldots p_{\sigma(k)}\right) \bar{p}_{1} \ldots \bar{p}_{\bar{k}-1}\right] \bar{p}_{\bar{k}} \\
= & a^{k}\left[\left(\sum_{\sigma \in \mathfrak{S}_{k}} p_{\sigma(1)} \ldots p_{\sigma(k)}\right) \bar{p}_{1} \ldots \bar{p}_{\bar{k}-1}\right] \bar{p}_{\bar{k}}
\end{aligned}
$$

using the inductive hypothesis at the second equality each time.

(c) Repeatedly applying the second line of (14):

$$
\begin{aligned}
& \operatorname{BRiffle} \tilde{\tau}_{a}^{-}\left(\bar{p}_{1} \bar{p}_{2} \ldots \bar{p}_{\bar{k}} \sum_{\sigma \in \mathfrak{S}_{k}} p_{\sigma(1)} \ldots p_{\sigma(k)}\right) \\
= & -\operatorname{BRiffle} \tilde{\tau}_{a}^{-}\left(\bar{p}_{2} \ldots \bar{p}_{\bar{k}} \sum_{\sigma \in \mathfrak{S}_{k}} p_{\sigma(1)} \ldots p_{\sigma(k)}\right) \bar{p}_{1} \\
= & \operatorname{BRiffle} \tilde{\tau}_{a}^{-}\left(\bar{p}_{3} \ldots \bar{p}_{\bar{k}} \sum_{\sigma \in \mathfrak{S}_{k}} p_{\sigma(1)} \ldots p_{\sigma(k)}\right) \bar{p}_{2} \bar{p}_{1} \\
& \vdots \\
= & (-1)^{\bar{k}} \operatorname{BRiffle} \tilde{\tau}_{a}^{-}\left(\sum_{\sigma \in \mathfrak{S}_{k}} p_{\sigma(1)} \ldots p_{\sigma(k)}\right) \bar{p}_{k} \ldots \bar{p}_{2} \bar{p}_{1} \\
= & (-1)^{\bar{k}} a^{k}\left(\sum_{\sigma \in \mathfrak{S}_{k}} p_{\sigma(1)} \ldots p_{\sigma(k)}\right) \bar{p}_{k} \ldots \bar{p}_{2} \bar{p}_{1},
\end{aligned}
$$


and by the same recursive process

$$
\begin{aligned}
& \text { BRiffle } \tilde{\tau}_{a}^{-}\left[\left(\sum_{\sigma \in \mathfrak{S}_{k}} p_{\sigma(1)} \ldots p_{\sigma(k)}\right) \bar{p}_{k} \ldots \bar{p}_{2} \bar{p}_{1}\right] \\
= & (-1)^{\bar{k}} a^{k} \bar{p}_{1} \bar{p}_{2} \ldots \bar{p}_{\bar{k}}\left(\sum_{\sigma \in \mathfrak{S}_{k}} p_{\sigma(1)} \ldots p_{\sigma(k)}\right) .
\end{aligned}
$$

Summing these gives the required eigenvector.

ii) Work in the subalgebra of $\mathcal{H}$ that is the universal enveloping algebra of $\operatorname{Prim}(\mathcal{H})$. As in the Triangularity Lemma (23), consider its PBW basis formed from the basis $\mathcal{P} \cup \overline{\mathcal{P}}$ of $\operatorname{Prim}(\mathcal{H})$, with the concatentation order on $\mathcal{P} \cup \overline{\mathcal{P}}$. Then, for each fixed format in part $\mathrm{i}$ of the Theorem, the eigenvector made from $p_{1}, \ldots, p_{k} \in \mathcal{P}$ and $\bar{p}_{1}, \ldots, \bar{p}_{\bar{k}} \in \overline{\mathcal{P}}$ is a sum of their products in different orders, and hence its highest length term in this PBW basis is $p_{1} \ldots p_{k} \bar{p}_{1} \ldots \bar{p}_{\bar{k}}$ (its coefficient is the number of product terms in the eigenvector, and is hence nonzero). Thus each eigenvector has a different highest length term, and so they are linearly independent.

iii) As explained above, each set of eigenvectors is triangular with respect to the PBW basis of the universal enveloping algebra of $\operatorname{Prim}(\mathcal{H})$, thus giving a basis of this universal enveloping algebra. By the Cartier-Milnor-Moore theorem (as described just before Notation 22), when $\mathcal{H}$ is cocommutative, this universal enveloping algebra is precisely $\mathcal{H}$.

Proof of Theorem 2\%. i) Proceed by induction on $\bar{k}$, the base case of $\bar{k}=0$ being Lemma 30. As noted in the previous proof, all lines in (14) are linear in $s$, so we may apply Lemma 31 when $s$ is the eigenvector in (12):

$$
\begin{aligned}
\text { BRiffle } \tau_{a}^{+}(\bar{p} s+s \bar{p}) & =\bar{p} \text { BRiffle } \tau_{a}^{+}(s)+\text { BRiffle } \tau_{a}^{+}(s) \bar{p} \\
& =\bar{p}\left(a^{k} s\right)+\left(a^{k} s\right) \bar{p}=a^{k}(\bar{p} s+s \bar{p}) ; \\
\text { BRiffle } \tau_{a}^{-}(\bar{p} s+s \bar{p}) & =-\bar{p} \operatorname{BRiffle} \tau_{a}^{-}(s)-\operatorname{BRiffle} \tau_{a}^{-}(s) \bar{p} \\
& =-\bar{p}(-1)^{\bar{k}} a^{k} s-(-1)^{\bar{k}} a^{k} s \bar{p} \\
& =(-1)^{\bar{k}+1} a^{k}(\bar{p} s+s \bar{p}) .
\end{aligned}
$$

So $\bar{p} s+s \bar{p}$ is an eigenvector with the required eigenvalue. It remains to show that, when $s$ is of the form (12), then so is $\bar{p} s+s \bar{p}$, with $\bar{k}$ increased by 1 . To do so, let 
$\bar{p}=\bar{p}_{\bar{k}+1}$. Then, for each set-composition $B$ of $\{1,2, \ldots, \bar{k}\}$ into 2 blocks,

$$
\begin{aligned}
& \bar{p}_{\bar{k}+1}\left(\coprod_{i \in B_{1}} \bar{p}_{i}\left(\sum_{\sigma \in \mathfrak{S}_{k}} p_{\sigma(1)} \ldots p_{\sigma(k)}\right) \prod_{i \in B_{2}} \bar{p}_{i}\right) \\
& +\left(\overleftarrow{\prod_{i \in B_{1}}} \bar{p}_{i}\left(\sum_{\sigma \in \mathfrak{S}_{k}} p_{\sigma(1)} \ldots p_{\sigma(k)}\right) \prod_{i \in B_{2}} \bar{p}_{i}\right) \bar{p}_{\bar{k}+1} \\
& =\overleftarrow{\prod_{i \in B_{1}^{\prime}}} \bar{p}_{i}\left(\sum_{\sigma \in \mathfrak{S}_{k}} p_{\sigma(1)} \ldots p_{\sigma(k)}\right) \prod_{i \in B_{2}^{\prime}} \bar{p}_{i}+\overleftarrow{\prod_{i \in B_{1}^{\prime \prime}}} \bar{p}_{i}\left(\sum_{\sigma \in \mathfrak{S}_{k}} p_{\sigma(1)} \ldots p_{\sigma(k)}\right) \prod_{i \in B_{2}^{\prime \prime}} \bar{p}_{i}
\end{aligned}
$$

where $B^{\prime}$ and $B^{\prime \prime}$ are set-compositions of $\{1,2, \ldots, \bar{k}+1\}$, obtained from $B$ respectively by adding $\bar{k}+1$ to $B_{1}$ or to $B_{2}$. And all set-compositions of $\{1,2, \ldots, \bar{k}+1\}$ into 2 blocks arise from a unique such $B$ in this way.

ii, iii. The same argument as in the proof of Theorem 26 above.

\section{Markov Chains from Hyperoctahedral Descent Operators}

One application of the eigenvalues and eigenvectors of hyperoctahedral descent operators calculated in previous sections is to study an associated Markov chain, generalising the type A framework in [DPR14, Pan18]. Each positive hyperoctahedral descent operator, applied to each basis of a Hopf algebra, determines a different Markov chain. We will be minimal here, and refer the reader to [Pan18] and [LPW09] for more background on Markov chains.

\subsection{Chain Construction}

Given a finite set $\mathcal{B}$ and a matrix $K$ with rows and columns labelled by elements of $\mathcal{B}$, the Markov chain with state space $\Omega$ and transition matrix $K$ is a sequence of random variables $X_{1}, X_{2}, \ldots$ taking values in $\mathcal{B}$, such that the conditional probability

$$
\operatorname{Prob}\left(X_{t}=y \mid X_{t-1}=x, X_{t-2}=x_{t-2}, \ldots, X_{1}=x_{1}\right)=\operatorname{Prob}\left(X_{t}=y \mid X_{t-1}=x\right)=K(x, y) .
$$

In other words, $X_{t}$, the state at time $t$, is only dependent on the state one timestep prior, not on further past history.

We would like this transition matrix $K$ to be (the transpose of) the matrix of a hyperoctahedral descent operator, acting on a graded Hopf algebra $\mathcal{H}$, relative to some fixed basis $\mathcal{B}$ (up to scaling). To be probabilities, the matrix entries must be non-negative; this motivates condition iv below, that the product and coproduct of basis elements expand positively in $\mathcal{B}$. Condition $\mathrm{v}$, the positivity of $\eta$, is required to scale the matrix so its rows sum to 1 , as probabilities should. Also, in condition ii, we require the involutive Hopf ambimorphism $\tau$ to send the basis $\mathcal{B}$ to itself (as opposed to to a linear combination of basis elements), so that it can be interpreted as an involution on the combinatorial objects indexing $\mathcal{B}$, analogous to flipping a deck of cards. 
Setup 33. The following conditions and notations will be assumed when analysing Markov chains driven by hyperoctahedral descent operators:

i) $\mathcal{H}=\bigoplus_{n \geqslant 0} \mathcal{H}_{n}$ is a graded connected Hopf algebra over $\mathbb{R}$, and each $\mathcal{H}_{n}$ is finitedimensional, with basis $\mathcal{B}_{n}$. And $\mathcal{B}$ denotes $\amalg_{n \geqslant 0} \mathcal{B}_{n}$.

ii) $\tau: \mathcal{B} \rightarrow \mathcal{B}$ is a graded involution that extends linearly to a Hopf ambimorphism on $\mathcal{H}$.

iii) $n$ is a fixed integer, and $P$ is a probability distribution on signed weak-compositions of $n$ or on tilde-signed weak-compositions of $n$ (but not a mixture of both). Define

$$
m \circ \Delta_{P(\tau)}:=\sum_{D} \frac{P(D)}{\left(\begin{array}{c}
n \\
D^{+}
\end{array}\right)} m \circ \Delta_{D(\tau)}
$$

where $\left(\begin{array}{c}n \\ D^{+}\end{array}\right)$is the multinomial coefficient $\left(\begin{array}{c}n \\ \left|d_{1}\right| \ldots\left|d_{l(D)}\right|\end{array}\right)$.

iv) For each signed or tilde-signed weak-composition $D$ with non-zero probability under $P$, and for all $x, y \in \mathcal{B}_{n}$, all $z_{1} \in \mathcal{B}_{\left|d_{1}\right|}, z_{2} \in \mathcal{B}_{\left|d_{2}\right|}, \ldots, z_{l(D)} \in \mathcal{B}_{\left|d_{l(D)}\right|}$ :

(a) $z_{1} z_{2} \ldots z_{l(D)}=\sum_{y \in \mathcal{B}_{n}} \xi_{z_{1}, \ldots, z_{l(D)}}^{y} y$ with $\xi_{z_{1}, \ldots, z_{l(D)}}^{y} \geqslant 0$;

(b) $\Delta_{D^{+}}(x)=\sum_{z_{i} \in \mathcal{B}_{\left|d_{i}\right|}} \eta_{x}^{z_{1}, \ldots, z_{l(D)}} z_{1} \otimes z_{2} \otimes \cdots \otimes z_{l(D)}$ with $\eta_{x}^{z_{1}, \ldots, z_{l(D)}} \geqslant 0$.

v) For all $x \in \mathcal{B}_{n}$, the function

$$
\eta(x):=\text { sum of coefficients (in the } \mathcal{B}_{1} \otimes \cdots \otimes \mathcal{B}_{1} \text { basis) of } \Delta_{1, \ldots, 1}(x)
$$

evaluates to a positive number.

Theorem 34. Under Setup 33, the matrix

$$
K(x, y):=\frac{\eta(y)}{\eta(x)} \text { coefficient of } y \text { in } m \circ \Delta_{P(\tau)}(x)
$$

is a transition matrix, and each step of the associated Markov chain, starting at $x \in \mathcal{B}_{n}$, is equivalent to the following four-step process:

1. Choose a signed or tilde-signed weak-composition $D$ according to the distribution $P$.

2. Choose $z_{1} \in \mathcal{B}_{\left|d_{1}\right|}, z_{2} \in \mathcal{B}_{\left|d_{2}\right|}, \ldots, z_{l(D)} \in \mathcal{B}_{\left|d_{l(D)}\right|}$ with probability

$$
\frac{1}{\eta(x)} \eta_{x}^{z_{1}, \ldots, z_{l(D)}} \eta\left(z_{1}\right) \ldots \eta\left(z_{l(D)}\right)
$$

3. For all $i$ such that $d_{i}$ is a decorated part, replace $z_{i}$ by $\tau\left(z_{i}\right)$. 
4. Choose $y \in \mathcal{B}_{n}$ with probability

$$
\left(\left(\begin{array}{l}
n \\
D
\end{array}\right) \eta\left(z_{1}\right) \ldots \eta\left(z_{l}\right)\right)^{-1} \xi_{z_{1}^{\prime}, \ldots, z_{l}^{\prime}}^{y} \eta(y)
$$

where $z_{i}^{\prime}=z_{i}$ if $d_{i}$ is an undecorated part, and $z_{i}^{\prime}=\tau\left(z_{i}\right)$ if $d_{i}$ is decorated.

We remark that (23) means $K$ is the transpose of the matrix for $m \circ \Delta_{P(\tau)}$, relative to the basis $\left\{\frac{x}{\eta(x)} \mid x \in \mathcal{B}_{n}\right\}$.

Proof. We follow the proof of [Pan18, Lem. 3.3]. According to [Pan18, Th. 2.3], to show $K$ is a transition matrix, it suffices to show that $\eta: \mathcal{B}_{n} \rightarrow \mathbb{R}$, extended linearly to $\mathcal{H}_{n}$, is an eigenvector of $\left(m \circ \Delta_{P(\tau)}\right)^{*}: \mathcal{H}^{*} \rightarrow \mathcal{H}^{*}$ of eigenvalue 1. Recall from Section 2.2 that $\left(m \circ \Delta_{P(\tau)}\right)^{*}=m \circ \Delta_{P\left(\tau^{*}\right)}$, where $\tau^{*}$ is an involution on the dual basis $\mathcal{B}^{*}$ of $\mathcal{H}^{*}$.

As in [Pan18, Lem. 3.3], let $\bullet * \in \mathcal{H}_{1}^{*}$ denote the linear map on $\mathcal{H}_{1}$ taking value 1 on each element of $\mathcal{B}_{1}$. When restricted to $\mathcal{H}_{n}, \eta=\left(\bullet^{*}\right)^{n} \in \mathcal{H}_{n}^{*}$. Since $\bullet^{*}$ is of degree 1 , it is necessarily primitive, so $\Delta_{D^{+}}\left(\left(\bullet^{*}\right)^{n}\right)=\left(\begin{array}{c}n \\ D^{+}\end{array}\right)\left(\bullet^{*}\right)^{d_{1}} \otimes \cdots \otimes\left(\bullet^{*}\right)^{d_{l}}$. Now $\bullet^{*}$ is the sum of all elements in $\mathcal{B}_{1}^{*}$, and $\tau^{*}$ is an involution on $\mathcal{B}_{1}^{*}$, so $\tau^{*}$ fixes $\bullet *$. As a Hopf ambimorphism, $\tau^{*}$ also fixes each $\left(\bullet^{*}\right)^{d_{i}}$, so $\Delta_{D}\left(\left(\bullet^{*}\right)^{n}\right)=\Delta_{D^{+}}\left(\left(\bullet^{*}\right)^{n}\right)=\left(\begin{array}{c}n \\ D^{+}\end{array}\right)\left(\bullet^{*}\right)^{d_{1}} \otimes \cdots \otimes\left(\bullet^{*}\right)^{d_{l}}$, and taking linear combination and then taking the product shows $m \circ \Delta_{P\left(\tau^{*}\right)}\left(\left(\bullet^{*}\right)^{n}\right)=\left(\left(\bullet^{*}\right)^{n}\right)$, as required.

The proof of the 4-step description of the chain is essentially the same as that of its type A version [Pan18, Th. 3.4], once we note that

$$
\eta\left(\tau\left(z_{i}\right)\right)=\left(\bullet^{*}\right)^{n}\left(\tau\left(z_{i}\right)\right)=\left[\tau^{*}\left(\bullet^{*}\right)^{n}\right]\left(z_{i}\right)=\left(\bullet^{*}\right)^{n}\left(z_{i}\right)=\eta\left(z_{i}\right),
$$

since $\tau^{*}$ fixes $(\bullet *)^{n}$.

\subsection{Stationary Distribution}

One basic question when studying a Markov chain is to find its stationary distributions, which are functions $\pi: \mathcal{B} \rightarrow \mathbb{R}$ satisfying

$$
\sum_{x \in \mathcal{B}} \pi(x) K(x, y)=\pi(y)
$$

These are of interest as they include all possible limiting distributions: if $\operatorname{Prob}\left(X_{t}=x\right)$ has a limit as $t \rightarrow \infty$, then this limit must be $\pi(x)$ for some stationary $\pi$.

To describe the stationary distributions of chains defined in (23), let $\mathcal{B}_{1}^{\tau}$ denote the subset of $\mathcal{B}_{1}$ that is fixed under $\tau$, and $\mathcal{B}_{1}^{-}$the set of averages over each size $2 \tau$-orbit in $\mathcal{B}_{1}$. (For example, in the signed shuffle algebra with $\tau$ defined as in $(2), \mathcal{B}_{1}^{-}$would contain $\frac{1}{2}(1+\overline{1}), \frac{1}{2}(2+\overline{2})$,etc., and $\mathcal{B}_{1}^{\tau}$ is empty.) Note that $\mathcal{B}_{1}^{-}$is also fixed under $\tau$; indeed, a basis for $\mathcal{H}_{1}^{\tau}$ is $\mathcal{B}_{1}^{\tau} \cup \mathcal{B}_{1}^{-}$. Given any multiset $\left\{c_{1}, \ldots, c_{n}\right\}$ in $\mathcal{B}_{1}^{\tau} \cup \mathcal{B}_{1}^{-}$, define $\pi_{c_{1}, \ldots, c_{n}}: \mathcal{B}_{n} \rightarrow \mathbb{R}$ by

$$
\pi_{c_{1}, \ldots, c_{n}}(x):=\frac{\eta(x)}{n !^{2}} \sum_{\sigma \in \mathfrak{S}_{n}} \text { coefficient of } x \text { in the product } c_{\sigma(1)} \ldots c_{\sigma(n)} .
$$


Note that, if $\mathcal{B}_{1}$ contains a sole element, denoted $\bullet$, then necessarily $\mathcal{B}_{1}^{\tau}=\{\bullet\}, \mathcal{B}_{1}^{-}=\varnothing$, so the only possible such function simplifies to

$$
\pi(x):=\frac{\eta(x)}{n !} \xi_{\bullet, \ldots, \bullet}^{x}
$$

Theorem 35. Assume the conditions in Setup 33, and additionally that $P$ is non-zero on some $D$ with at least two non-zero parts, and on some $D$ with at least one decorated part. Then any stationary distribution for the Markov chain defined in Equation (23) is a unique linear combination of the $\pi_{c_{1}, \ldots, c_{n}}$, over all multisets $\left\{c_{1}, \ldots, c_{n}\right\}$ in $\mathcal{B}_{1}^{\tau} \cup \mathcal{B}_{1}^{-}$. Furthermore, $\sum_{x \in \mathcal{B}_{n}} \pi_{c_{1}, \ldots, c_{n}}(x)=1$. In particular, if $\mathcal{B}_{1}=\{\bullet\}$, then $\pi$ is the unique stationary distribution.

Proof. Recall that $K$ is the transpose of the matrix for $m \circ \Delta_{P(\tau)}$, relative to the basis $\left\{\frac{x}{\eta(x)} \mid x \in \mathcal{B}_{n}\right\}$. Thus the condition of $(24)$ translates to $\frac{1}{n !^{2}} \sum_{\sigma \in \mathfrak{S}_{n}} c_{\sigma(1)} \ldots c_{\sigma(n)}$ being an eigenvector of eigenvalue 1 for $m \circ \Delta_{P(\tau)}$. To check this, recall $c_{i} \in \mathcal{B}_{1}^{\tau} \cup \mathcal{B}_{1}^{-} \subseteq \operatorname{Prim}(H)^{\tau}$, so by $(10)$

$$
m \circ \Delta_{D(\tau)}\left(c_{\sigma(1)} \ldots c_{\sigma(n)}\right)=\sum_{B_{1}, \ldots, B_{l(D)}}\left(\prod_{i \in B_{1}} c_{\sigma(i)}\right) \ldots\left(\prod_{i \in B_{l(D)}} c_{\sigma(i)}\right)
$$

summing over all set-compositions $B$ compatible with $\lambda=(1, \ldots, 1), \bar{\lambda}=\varnothing, D$. Each summand on the right is a product of $c_{\sigma(1)}, \ldots, c_{\sigma(n)}$ in some order, and there are $\left(\begin{array}{c}n \\ D^{+}\end{array}\right)$ terms, so when symmetrised, $\sum_{\sigma \in \mathfrak{S}_{n}} c_{\sigma(1)} \ldots c_{\sigma(n)}$ is an eigenvector of eigenvalue $\left(\begin{array}{c}n \\ D^{+}\end{array}\right)$for $m \circ \Delta_{D(\tau)}$. Taking the linear combination of hyperoctahedral descent operators as in (22) gives the result.

The Theorem further claims that these $\frac{1}{n !^{2}} \sum_{\sigma \in \mathfrak{S}_{n}} c_{\sigma(1)} \ldots c_{\sigma(n)}$ give a basis of the eigenspace of eigenvalue 1 for $m \circ \Delta_{P(\tau)}$, as $\left\{c_{1}, \ldots, c_{n}\right\}$ ranges over all multisets in $\mathcal{B}_{1}^{\tau} \cup \mathcal{B}_{1}^{-}$. To see the linear independence, note that, if the basis $\mathcal{P}$ of $(\text { Prim } \mathcal{H})^{\tau}$ contains $\mathcal{B}_{1}^{\tau} \cup \mathcal{B}_{1}^{-}$, then the PBW basis constructed in and before Notation 22 contains $c_{1} \ldots c_{n}$. Hence, after PBW straightening, the highest length term in $\frac{1}{n !} \sum_{\sigma \in \mathfrak{S}_{n}} c_{\sigma(1)} \ldots c_{\sigma(n)}$ is $c_{1} \ldots c_{n}$, a different term for each choice of multiset $\left\{c_{1}, \ldots, c_{n}\right\}$ in $\mathcal{B}_{1}^{\tau} \cup \mathcal{B}_{1}^{-}$, showing linear independence. To see that these elements span the eigenspace of eigenvalue 1 , we separate into two claims as in the type A case [Pan18, Th. 3.12]:

i) the only double-partition with $\sum_{D} \frac{P(D)}{\left(\begin{array}{c}n \\ D^{+}\end{array}\right)} \beta_{\lambda, \bar{\lambda}}^{D}=1$ is $\lambda=(1, \ldots, 1), \bar{\lambda}=\varnothing-$ in the proof of $\left[\right.$ Pan18, Th. 3.12], it was shown that $\beta_{\lambda, \varnothing}^{D} \leqslant\left(\begin{array}{c}n \\ D^{+}\end{array}\right)$with equality if and only if $D$ has a single non-zero part or $\lambda=(1, \ldots, 1)$. (In short, this is because $\beta_{(1, \ldots, 1), \varnothing}^{D}$ by definition counts the set-compositions of $\{1,2, \ldots, n\}$ into $l(D)$ blocks such that block $i$ contains $\left|d_{i}\right|$ elements, and an alternate view of $\beta_{\lambda, \varnothing}^{D}$ is that it counts such set-compositions with the extra condition that $1,2, \ldots, \lambda_{1}$ are in the same block, $\lambda_{1}+1, \lambda_{1}+2, \ldots, \lambda_{1}+\lambda_{2}$ are in the same block, and so on.) Now $\beta_{\lambda, \bar{\lambda}}^{D}$ and $\beta_{\lambda \cup \bar{\lambda}, \varnothing}^{D}$ are respectively a signed and unsigned enumeration of the same set-compositions, 
so $\beta_{\lambda, \bar{\lambda}}^{D} \leqslant \beta_{\lambda \cup \bar{\lambda}, \varnothing}^{D}$ with equality if and only if $\bar{\lambda}=\varnothing$ or, in all set-compositions compatible with $\lambda, \bar{\lambda}, D$, the signed integers $\bar{i}$ can only be in blocks corresponding to unsigned parts of $D$. In particular, if $\lambda \cup \bar{\lambda}=(1, \ldots, 1)$, then this latter condition can only happen if $D$ has no decorated parts. So $\beta_{\lambda, \bar{\lambda}}^{D} \leqslant \beta_{\lambda \cup \bar{\lambda}, \varnothing}^{D} \leqslant\left(\begin{array}{c}n \\ D^{+}\end{array}\right)$, with equality if and only if $D$ has a single non-zero part, or $\lambda=\bar{\lambda}=(1, \ldots, 1)$ and $D$ has no decorated parts, or $\lambda=(1, \ldots, 1)$ and $\bar{\lambda}=\varnothing$. The hypotheses in Theorem 35 exactly rules out the first two equality cases for some $D$ in the sum $\sum_{D} \frac{P(D)}{\left(\begin{array}{c}n \\ D^{+}\end{array}\right)} \beta_{\lambda, \bar{\lambda}}^{D}$, so $\sum_{D} \frac{P(D)}{\left(\begin{array}{c}n \\ D^{+}\end{array}\right)} \beta_{\lambda, \bar{\lambda}}^{D} \leqslant 1$ with equality if and only if $\lambda=(1, \ldots, 1), \bar{\lambda}=\varnothing$.

ii) the multiplicity of the eigenvalue

$$
\sum_{D} \frac{P(D)}{\left(\begin{array}{c}
n \\
D^{+}
\end{array}\right)} \beta_{(1, \ldots, 1), \varnothing}^{D}
$$

is the number of multisets $\left\{c_{1}, \ldots, c_{n}\right\}$ in $\mathcal{B}_{1}^{\tau} \cup \mathcal{B}_{1}^{-}$- by Theorem 17 , the required multiplicity is $\left(\begin{array}{c}\boldsymbol{b} \\ (1, \ldots, 1)\end{array}\right)\left(\begin{array}{l}\overline{\boldsymbol{b}} \\ \varnothing\end{array}\right)=\left(\begin{array}{c}b_{1}+n-1 \\ n\end{array}\right)$. From (8) and (9) defining the sequences $\boldsymbol{b}$ and $\overline{\boldsymbol{b}}$, it is clear that $b_{1}=\operatorname{dim} \mathcal{H}_{1}^{\tau}=\left|\mathcal{B}_{1}^{\tau} \cup \mathcal{B}_{1}^{-}\right|$, hence $\left(\begin{array}{c}b_{1}+n-1 \\ n\end{array}\right)$ is the number of $n$-element multisets in $\mathcal{B}_{1}^{\tau} \cup \mathcal{B}_{1}^{-}$.

Finally, we check $\sum_{x \in \mathcal{B}_{n}} \pi_{c_{1}, \ldots, c_{n}}(x)=1$. The key is the following identity, from the proof of the type A case [Pan18, Th. 3.12]:

$$
\sum_{x \in \mathcal{B}_{n}} \eta(x) \xi_{d_{1}, d_{2}, \ldots, d_{n}}^{x}=n \text { !, for any choice of } d_{1}, \ldots, d_{n} \in \mathcal{B}_{1}
$$

Now, given multisets $\left\{c_{1}, \ldots, c_{k}\right\} \subseteq \mathcal{B}_{1}^{\tau}$ and $\left\{c_{k+1}, \ldots, c_{n}\right\} \subseteq \mathcal{B}_{1}^{-}$, let $c_{i}=\frac{1}{2}\left(d_{i}+d_{i}^{\prime}\right)$ for $i>k$, with $d_{i}, d_{i}^{\prime} \in \mathcal{B}_{1}$. Then the coefficient of $x$ in $c_{1} \ldots c_{n}$ is

$$
\begin{aligned}
& \frac{1}{2^{n-k}}\left(\xi_{c_{1}, \ldots, c_{k}, d_{k+1}, \ldots, d_{n}}^{x}+\xi_{c_{1}, \ldots, c_{k}, d_{k+1}^{\prime}, \ldots, d_{n}}^{x}\right. \\
& \left.\quad+\xi_{c_{1}, \ldots, c_{k}, d_{k+1}, d_{k+2}^{\prime}, \ldots, d_{n}}^{x}+\xi_{c_{1}, \ldots, c_{k}, d_{k+1}^{\prime}, d_{k+2}^{\prime}, \ldots, d_{n}}^{x}+\ldots\right)
\end{aligned}
$$

with $2^{n-k}$ terms in total, running through each combination of $d_{i}$ or $d_{i}^{\prime}$. Each of these $2^{n-k}$ terms, when multiplied by $\eta(x)$ and summed over $x \in \mathcal{B}_{n}$, gives $n$ ! by (25), and the same is true when considering the coefficient of $x$ in $c_{\sigma(1)} \ldots c_{\sigma(n)}$ for all $\sigma \in \mathfrak{S}_{n}$.

\section{Applications to Hyperoctahedral Riffle-Shuffling}

This section applies the eigenvector formulas of Section 3.2 to analyse hyperoctahedral riffle-shuffles, as defined in Section 5.1 below. Recall that each formula depends on a basis of primitives, which Section 5.2 will describe, before full computation of the eigenbasis in Section 5.3 and its application to deduce the expected number of descents and the probability of having sign equal to 1 in Sections 5.5 and 5.6. 


\subsection{Hyperoctahedral Riffle-Shuffles}

For the rest of the paper, we study two families of hyperoctahedral riffle-shuffles, the a-handed riffle-shuffle with rotation and a-handed riffle-shuffle with flip. These are essentially the Markov chains described in Theorem 34, when $\mathcal{H}$ is the signed shuffle algebra of Example 1, $\tau$ and $\tilde{\tau}$ are as defined in (2) and (3), and $m \circ \Delta_{P(\tau)}=\frac{1}{a^{n}}$ BRiffle $\tau_{a}^{+}$or $\frac{1}{a^{n}}$ BRiffle $\tau_{a}^{-}$(for rotation), or $\frac{1}{a^{n}}$ BRiffle $\tilde{\tau}_{a}^{+}$or $\frac{1}{a^{n}}$ BRiffle $\tilde{\tau}_{a}^{-}$(for flip). The factor of $\frac{1}{a^{n}}$ is necessary in order that the resulting operator can be written in the form in (22), for a probability distribution $P$ that sums to 1 . For example, for $\frac{1}{a^{n}}$ BRiffle $\tau_{a}^{+}$, the distribution is $P(D)=\left(\begin{array}{c}n \\ D^{+}\end{array}\right)$for all $D$ with $a$ parts and odd parts positive, even parts negative; and $P(D)=0$ otherwise. The case for the other three operators are similar.

We take the state space $\mathcal{B}_{n}$ to be all $2^{n} n$ ! ways of ordering $n$ distinct cards and tracking their orientations. Algebraically, $\mathcal{B}_{n}$ is the set of words of length $n$, where, for each $i \in\{1,2, \ldots, n\}, i$ or $\bar{i}$ appear exactly once. We may call these signed permutations of $n$, viewed in one-line notation. Technically, $\mathcal{B}_{n}$ is not a basis of $\mathcal{H}_{n}$, which should include decks with repeated cards (words with repeated letters), but the span of $\mathcal{B}_{n}$ is invariant under $m \circ \Delta_{P(\tau)}$ and $m \circ \Delta_{P(\tilde{\tau})}$ for all $P$, so the theory of the previous sections can apply with minor modifications. Note that $\eta(x)=1$ for all $x \in \mathcal{B}_{n}$.

The application of Theorem 34 to this situation shows:

Corollary 36. Fix an integer $n$, and let $x, y$ be signed permutations of $n$. For a fixed integer a, the matrix

$$
\left.K(x, y):=\text { coefficient of } y \text { in } \frac{1}{a^{n}} \text { BRiffle } \tau_{a}^{+}(x) \text { (resp. } \frac{1}{a^{n}} \text { BRiffle } \tau_{a}^{-}(x)\right)
$$

is the transition matrix for the following card shuffle:

1. Choose a weak-composition $\left(d_{1}, \ldots, d_{a}\right)$ of $n$ according to the multinomial distribution, i.e. with probability $\frac{1}{a^{n}}\left(\begin{array}{ccc} & n & \\ d_{1} & \ldots & d_{a}\end{array}\right)$.

2. Cut the deck into a piles so the ith pile contains $d_{i}$ cards.

3. Rotate the first, third, fifth, ...piles (resp. second, fourth, sixth, ...piles) by 180 degrees.

4. Uniformly choose one of the $\left(\begin{array}{ccc}n & \\ d_{1} & \ldots & d_{a}\end{array}\right)$ interleavings of the a piles. Equivalently, drop the cards one-by-one from the bottom of one of the a piles, chosen with probability proportional to the current pile size.

If we use $\tilde{\tau}$ in place of $\tau$, then Step 3 should flip the piles upside down instead of rotating.

The equivalence in step 4 is proved in [BD92, Lem. 1] regarding riffle-shuffles.

Recall that, in type A, a $b$-handed riffle-shuffle followed by an $a$-handed shuffle is an $a b$-handed shuffle. By Proposition 24.iii, this is true for shuffles with flip, composing the 
signs in BRiffle $\tilde{\tau}_{a}^{+}$and BRiffle $\tilde{\tau}_{a}^{-}$in the natural way. However, for shuffles with rotation, Proposition 24.i asserts that $a$, the number of hands in the second shuffle, must be odd.

We would like to compute the expected value of some functions under this shuffle, using the eigenvectors of the hyperoctahedral descent operators as proved in Section 3.2. By Propositions 2.1 and 2.4 of [Pan18] (reproduced below), such calculations should take place on the dual of the algebra defining the chain, namely the signed free associative algebra in our case.

Proposition 37. [Pan18, Prop. 2.1, 2.4] Suppose $K$ is the transition matrix for the Markov chain $\left\{X_{t}\right\}$ on the state space $\mathcal{B}$. Let $\mathbf{f}: \mathcal{B} \rightarrow \mathbb{R}$ be a (right) eigenfunction of $\left\{X_{t}\right\}$ with eigenvalue $\beta$, meaning $\sum_{y \in \mathcal{B}} K(x, y) \mathbf{f}(y)=\beta \mathbf{f}(x)$. Then the expected value of $\mathbf{f}$ is

$$
\operatorname{Expect}\left(\mathbf{f}\left(X_{t}\right) \mid X_{0}=x_{0}\right):=\sum_{y \in \mathcal{B}} K^{t}\left(x_{0}, y\right) \mathbf{f}(y)=\beta^{t} \mathbf{f}\left(x_{0}\right)
$$

In the particular case where $\left\{X_{t}\right\}$ arises from the construction of Theorem 34, the right eigenfunctions $\mathbf{f}$ are in bijection with the eigenvectors $f \in \mathcal{H}^{*}$ of the dual map $m \circ \Delta_{P\left(\tau^{*}\right)}$, through the vector space isomorphism

$$
\mathbf{f}(x):=\frac{1}{\eta(x)} f(x)
$$

\subsection{Lyndon Word Combinatorics for Signed Words}

Let $\mathcal{A}$ be an ordered alphabet, and $T(\mathcal{A})$ the free associative algebra over $\mathcal{A}$. The usual basis for Prim $(T(\mathcal{A}))$ are the "standard-bracketings of Lyndon words in $\mathcal{A}$ " [Lot97, Ch. 5]. The following explains this when $\mathcal{A}=\{\overline{1} \preceq 1 \preceq \overline{2} \preceq 2 \preceq \cdots \preceq \bar{N} \preceq N\}$, with an important modification so that the output will be either $\tau$-invariant or $\tau$-negating, and hence suitable for input into the eigenvector formulas of Theorems 26 and 27.

A word is Lyndon if it is lexicographically smaller than all its cyclic rearrangements. For example, $\overline{1} 6 \overline{7} \overline{2}$ is Lyndon, because $\overline{1} 6 \overline{7} \overline{2} \prec 6 \overline{7} \overline{2} \overline{1}, \overline{7} \overline{2} \overline{1} 6, \overline{2} \overline{1} 6 \overline{7}$. In contrast, $\overline{5} 3$ and $3 \overline{5} 3 \overline{5}$ are not Lyndon.

Remark. A word with distinct letters is Lyndon if and only if its starting letter is minimal amongst all its letters.

Definition 38. Given a Lyndon word $u$, its signed standard-bracketing $\operatorname{stdbrac}(u)$ is computed recursively as follows: if $u=i$ is a single positive letter, $\operatorname{set} \operatorname{stdbrac}(u):=i+\bar{i}$. If $u=\bar{i}$ is a single negative letter, $\operatorname{set} \operatorname{stdbrac}(u):=i-\bar{i}$. Otherwise, write $u$ as the concatentation of Lyndon words $\overleftarrow{u}$ and $\vec{u}$ both non-empty such that $\vec{u}$ is of maximal length - [Lot97, Prop. 5.1.3] asserts that this "standard factorisation" is possible. Then define

$$
\operatorname{stdbrac}(u):=[\operatorname{stdbrac}(\overleftarrow{u}), \operatorname{stdbrac}(\vec{u})]
$$

(These brackets denote the Lie bracket, $[z, w]:=z w-w z$.)

Remark. From the previous Remark, it is easy to see that, if $u$ has distinct letters, then $\vec{u}$ begins precisely with the second-minimal letter of $u$. 


\section{Example 39.}

$$
\begin{aligned}
\operatorname{stdbrac}(\overline{1} 6 \overline{7} \overline{2}):= & \operatorname{stdbrac}(\overline{1} 6 \overline{7}), \operatorname{stdbrac}(\overline{2})] \\
= & \operatorname{stdbrac}(\overline{1} 6 \overline{7}), 2-\overline{2}] \\
= & {[[1-\overline{1}, \operatorname{stdbrac}(6 \overline{7})], 2-\overline{2}] } \\
= & {[[1-\overline{1},[6+\overline{6}, 7-\overline{7}]], 2-\overline{2}] } \\
= & 672-16 \overline{7} 2+1 \overline{6} 72-1 \overline{6} \overline{7} 2-1762-17 \overline{6} 2+1 \overline{7} 62+1 \overline{7} \overline{6} 2 \\
& -\overline{1} 672+\overline{1} 6 \overline{7} 2-\overline{1} \overline{6} 72+\overline{1} \overline{6} \overline{7} 2+\overline{1} 762+\overline{1} 7 \overline{6} 2-\overline{1} \overline{7} 62-\overline{1} \overline{7} \overline{6} 2 \\
& -6712+6 \overline{7} 12-\overline{6} 712+\overline{6} \overline{7} 12+7612+7 \overline{6} 12-\overline{7} 612-\overline{7} \overline{6} 12 \\
& +67 \overline{1} 2-6 \overline{7} \overline{1} 2+\overline{6} 7 \overline{1} 2-\overline{6} \overline{1} \overline{1} 2-76 \overline{1} 2-7 \overline{6} \overline{1} 2+\overline{7} 6 \overline{1} 2+\overline{7} \overline{6} \overline{1} 2 \\
- & 167 \overline{2}+16 \overline{7} \overline{2}-1 \overline{6} 7 \overline{2}+1 \overline{6} \overline{7} \overline{2} \\
& +\ldots(28 \text { more terms, analogous to those above) } \\
& -2167+216 \overline{7}-21 \overline{6} 7+21 \overline{6} \overline{7}+\ldots \text { (28 more terms) } \\
+ & \overline{2} 167-\overline{2} 16 \overline{7}+\overline{2} 1 \overline{6} 7-\overline{2} 1 \overline{6} \overline{7}+\ldots \text { (28 more terms). }
\end{aligned}
$$

Proposition 40 and Corollary 41 explain how to determine if $\operatorname{stdbrac}(u)$ is $\tau$-invariant or $\tau$-negating.

Proposition 40. Let $\mathcal{H}$ be any Hopf algebra.

For $\tau: \mathcal{H} \rightarrow \mathcal{H}$ a linear involution that is an algebra morphism,

$$
\left[\mathcal{H}^{\tau}, \mathcal{H}^{\tau}\right] \subseteq \mathcal{H}^{\tau}, \quad\left[\mathcal{H}^{\tau}, \mathcal{H}^{-\tau}\right] \subseteq \mathcal{H}^{-\tau}, \quad\left[\mathcal{H}^{-\tau}, \mathcal{H}^{-\tau}\right] \subseteq \mathcal{H}^{\tau} .
$$

For $\tilde{\tau}: \mathcal{H} \rightarrow \mathcal{H}$ a linear involution that is an algebra antimorphism,

$$
\left[\mathcal{H}^{\tilde{\tau}}, \mathcal{H}^{\tilde{\tau}}\right] \subseteq \mathcal{H}^{-\tilde{\tau}}, \quad\left[\mathcal{H}^{\tilde{\tau}}, \mathcal{H}^{-\tilde{\tau}}\right] \subseteq \mathcal{H}^{\tilde{\tau}}, \quad\left[\mathcal{H}^{-\tilde{\tau}}, \mathcal{H}^{-\tilde{\tau}}\right] \subseteq \mathcal{H}^{-\tilde{\tau}}
$$

Proof. For $x, y \in \mathcal{H}^{\tau}$ and $\bar{x}, \bar{y} \in \mathcal{H}^{-\tau}$ :

$$
\begin{aligned}
& \tau([x, y])=\tau(x y-y x)=\tau(x) \tau(y)-\tau(y) \tau(x)=x y-y x=[x, y] \\
& \tau([x, \bar{y}])=\tau(x \bar{y}-\bar{y} x)=\tau(x) \tau(\bar{y})-\tau(\bar{y}) \tau(x)=x(-\bar{y})-(-\bar{y}) x=-[x, \bar{y}] ; \\
& \tau([\bar{x}, \bar{y}])=\tau(\bar{x} \bar{y}-\bar{y} \bar{x})=\tau(\bar{x}) \tau(\bar{y})-\tau(\bar{y}) \tau(\bar{x})=(-\bar{x})(-\bar{y})-(-\bar{y})(-\bar{x})=[\bar{x}, \bar{y}] .
\end{aligned}
$$

For $x, y \in \mathcal{H}^{\tilde{\tau}}$ and $\bar{x}, \bar{y} \in \mathcal{H}^{-\tilde{\tau}}$ :

$$
\begin{aligned}
& \tilde{\tau}([x, y])=\tilde{\tau}(x y-y x)=\tilde{\tau}(y) \tilde{\tau}(x)-\tilde{\tau}(x) \tilde{\tau}(y)=y x-x y=-[x, y] \\
& \tilde{\tau}([x, \bar{y}])=\tilde{\tau}(x \bar{y}-\bar{y} x)=\tilde{\tau}(\bar{y}) \tilde{\tau}(x)-\tilde{\tau}(x) \tilde{\tau}(\bar{y})=(-\bar{y}) x-x(-\bar{y})=[x, \bar{y}] \\
& \tilde{\tau}([\bar{x}, \bar{y}])=\tilde{\tau}(\bar{x} \bar{y}-\bar{y} \bar{x})=\tilde{\tau}(\bar{y}) \tilde{\tau}(\bar{x})-\tilde{\tau}(\bar{x}) \tilde{\tau}(\bar{y})=(-\bar{y})(-\bar{x})+(-\bar{x})(-\bar{y})=[\bar{x}, \bar{y}] .
\end{aligned}
$$

Corollary 41. Let $u$ be a Lyndon word in the alphabet $\mathcal{A}=\{\overline{1} \preceq 1 \preceq \overline{2} \preceq 2 \preceq \cdots \preceq$ $\bar{N} \preceq N\}$, and $T(\mathcal{A})$ be the free associative algebra over $\mathcal{A}$, i.e. the signed free associative algebra. Then

$\operatorname{stdbrac}(u) \in(\operatorname{Prim} T(\mathcal{A}))^{\tau} \Longleftrightarrow u$ has an even number of negative letters; $\operatorname{stdbrac}(u) \in(\operatorname{PrimT}(\mathcal{A}))^{-\tau} \Longleftrightarrow u$ has an odd number of negative letters; $\operatorname{stdbrac}(u) \in(\operatorname{Prim} T(\mathcal{A}))^{\tilde{\tau}} \Longleftrightarrow u$ has an odd number of positive letters; $\operatorname{stdbrac}(u) \in(\operatorname{PrimT}(\mathcal{A}))^{-\tilde{\tau}} \Longleftrightarrow u$ has an even number of positive letters. 
Proof. We prove the last line. The other three are entirely analogous.

Apply induction on the length of $u$. In the base case where $u$ is a single letter, then it has an even number of positive letters precisely when $u$ is a negative letter, e.g. $u=\bar{i}$. Then $\operatorname{stdbrac}(u)=i-\bar{i} \in(\operatorname{Prim} T(\mathcal{A}))^{-\tilde{\tau}}$ as required.

Now suppose $u$ has at least two letters. If $u$ has an even number of positive letters, then there are two possibilities for $\overleftarrow{u}$ and $\vec{u}$

- $\overleftarrow{u}$ and $\vec{u}$ both have an odd number of positive letters, so by inductive hypothesis $\operatorname{stdbrac}(\overleftarrow{u}), \operatorname{stdbrac}(\vec{u}) \in(\operatorname{Prim} T(\mathcal{A}))^{\tilde{\tau}}$. So

$$
\begin{aligned}
\operatorname{stdbrac}(u) & =[\operatorname{stdbrac}(\overleftarrow{u}), \operatorname{stdbrac}(\vec{u})] \\
& \subseteq\left[(\operatorname{Prim} T(\mathcal{A}))^{\tilde{\tau}},(\operatorname{PrimT}(\mathcal{A}))^{\tilde{\tau}}\right] \subseteq(\operatorname{PrimT}(\mathcal{A}))^{-\tilde{\tau}}
\end{aligned}
$$

using Proposition 40 for the last inclusion.

- $\overleftarrow{u}$ and $\vec{u}$ both have an even number of positive letters, so $\operatorname{stdbrac}(\overleftarrow{u}), \operatorname{stdbrac}(\vec{u}) \in$ $(\operatorname{Prim} T(\mathcal{A}))^{-\tilde{\tau}}$. So

$$
\operatorname{stdbrac}(u) \in\left[(\operatorname{PrimT}(\mathcal{A}))^{-\tilde{\tau}},(\operatorname{Prim} T(\mathcal{A}))^{-\tilde{\tau}}\right] \subseteq(\operatorname{PrimT}(\mathcal{A}))^{-\tilde{\tau}}
$$

also.

\subsection{Eigenbasis Algorithm}

The previous section gave a signed standard-bracketing algorithm which converts each Lyndon word to a $\tau$-invariant or $\tau$-negating primitive. Theorems 26 and 27 associate an eigenvector for each multiset of such primitives, but it's more convenient to index the eigenvectors by words. The latter indexing may be achieved by a bijection sending a word $w$ to its decreasing Lyndon factorisation $\left(u_{1}, \ldots, u_{k^{\prime}}\right)$, where $w$ is the concatenation of the Lyndon words $u_{i}$ satisfying $u_{1} \succeq u_{2} \succeq \cdots \succeq u_{k^{\prime}}$ in lexicographic order. By the Remark before Definition 38, if $w$ has distinct letters, then the $u_{i}$ begin at precisely the left-to-right minima of $w$. For example, the decreasing Lyndon factorisation of $\overline{4} 35 \overline{1} 6 \overline{7} \overline{2}$ is $(\overline{4}, 35, \overline{1} 6 \overline{7} \overline{2})$.

Hence the full eigenvector algorithm is as follows:

Algorithm 42. To associate to a word $w$ an eigenvector of BRiffle $\tau_{a}^{+}$or BRiffle $\tau_{a}^{-}$for odd $a$, or of BRiffle $\tilde{\tau}_{a}^{+}$or BRiffle $\tilde{\tau}_{a}^{-}$for any $a$, on the signed free associative algebra:

1. Take the decreasing Lyndon factorisation $u_{1}, \ldots, u_{k^{\prime}}$ of $w$.

2. Calculate $\operatorname{stdbrac}\left(u_{i}\right)$ for each Lyndon factor using Definition 38 .

3. Use Corollary 41 to determine whether each result from Step 2 is $\tau$-invariant or $\tau$-negating - relabel the $\tau$-invariant ones as $p_{1}, \ldots, p_{k}$ and the $\tau$-negating ones as $\bar{p}_{1} \ldots, \bar{p}_{\bar{k}}$.

4. Assemble the $p_{i}$ and $\bar{p}_{i}$ according to Theorem 26 or 27 for the desired operator. 
By Theorems 26.iii and 27.iii, the eigenvectors associated with all words in the above manner form a basis of the free associative algebra $\mathcal{H}$.

Example 43. We calculate the eigenvector for BRiffle $\tau_{3}^{+}$corresponding to $w=\overline{4} 35 \overline{1} 6 \overline{7} \overline{2}$.

1. The Lyndon factors of $w$ are $u_{1}=\overline{4}, u_{2}=35, u_{3}=\overline{1} 6 \overline{7} \overline{2}$.

2. Their standard bracketings are, respectively, $4-\overline{4},[3+\overline{3}, 5+\overline{5}]$ and $[[1-\overline{1},[6+$ $\overline{6}, 7-\overline{7}]], 2-\overline{2}]$.

3. $\overline{4}$ has one negative letter, so $4-\overline{4}$ is $\tau$-negating. Call this $\bar{p}_{1}$.

35 has no negative letters, so $[3+\overline{3}, 5+\overline{5}]$ is $\tau$-invariant. Call this $p_{1}$.

$\overline{1} 6 \overline{7} \overline{2}$ has three negative letters, so $[[1-\overline{1},[6+\overline{6}, 7-\overline{7}]], 2-\overline{2}]$ is $\tau$-negating. Call this $\bar{p}_{2}$.

4. Since $a=3$ is odd, Theorem 27 applies. By inputting $p_{1}, \bar{p}_{1}, \bar{p}_{2}$ above into (12):

$$
\begin{aligned}
& (4-\overline{4})[[1-\overline{1},[6+\overline{6}, 7-\overline{7}]], 2-\overline{2}][3+\overline{3}, 5+\overline{5}] \\
+ & (4-\overline{4})[3+\overline{3}, 5+\overline{5}][[1-\overline{1},[6+\overline{6}, 7-\overline{7}]], 2-\overline{2}] \\
+ & {[[1-\overline{1},[6+\overline{6}, 7-\overline{7}]], 2-\overline{2}][3+\overline{3}, 5+\overline{5}](4-\overline{4}) } \\
+ & {[3+\overline{3}, 5+\overline{5}][[1-\overline{1},[6+\overline{6}, 7-\overline{7}]], 2-\overline{2}](4-\overline{4}) }
\end{aligned}
$$

is an eigenvector for BRiffle $\tau_{3}^{+}$, of eigenvalue 3 .

Example 44. We calculate the eigenvector for BRiffle $\tilde{\tau}_{2}^{+}$and BRiffle $\tilde{\tau}_{3}^{-}$corresponding to $w=\overline{4} \overline{3} 5 \overline{1} 6 \overline{7} \overline{2}$. Steps 1 and 2 are similar to Example 43 above.

3. $\overline{4}$ has no positive letters, so $4-\overline{4}$ is $\tilde{\tau}$-negating. Call this $\bar{p}_{1}$.

$\overline{3} 5$ has one positive letter, so $[3-\overline{3}, 5+\overline{5}]$ is $\tilde{\tau}$-invariant. Call this $p_{1}$.

$\overline{1} 6 \overline{7} \overline{2}$ has one positive letter, so $[[1-\overline{1},[6+\overline{6}, 7-\overline{7}]], 2-\overline{2}]$ is $\tilde{\tau}$-invariant. Call this $p_{2}$.

4. Since $a=2$ is even, input $p_{1}, p_{2}, \bar{p}_{1}$ above into the second formula in Theorem 26.ia:

$$
\begin{aligned}
(4-\overline{4})([3-\overline{3}, 5+\overline{5}][[1-\overline{1},[6+\overline{6}, 7-\overline{7}]], 2-\overline{2}] \\
+[3-\overline{3}, 5+\overline{5}][[1-\overline{1},[6+\overline{6}, 7-\overline{7}]], 2-\overline{2}])
\end{aligned}
$$

is an eigenvector for BRiffle $\tilde{\tau}_{2}^{+}$, of eigenvalue 0 . And, for BRiffle $\tilde{\tau}_{3}^{-}$, input $p_{1}, p_{2}, \bar{p}_{1}$ into Theorem 26.ic:

$$
\begin{aligned}
& (4-\overline{4})([3-\overline{3}, 5+\overline{5}][[1-\overline{1},[6+\overline{6}, 7-\overline{7}]], 2-\overline{2}] \\
& +[3-\overline{3}, 5+\overline{5}][[1-\overline{1},[6+\overline{6}, 7-\overline{7}]], 2-\overline{2}]) \\
& +([3-\overline{3}, 5+\overline{5}][[1-\overline{1},[6+\overline{6}, 7-\overline{7}]], 2-\overline{2}] \\
& \quad+[3-\overline{3}, 5+\overline{5}][[1-\overline{1},[6+\overline{6}, 7-\overline{7}]], 2-\overline{2}])(4-\overline{4})
\end{aligned}
$$

is an eigenvector of eigenvalue -3 . 


\begin{tabular}{|c|c|c|c|}
\hline $\begin{array}{c}\text { parity } \\
\text { of } a\end{array}$ & shuffle & eigenvalue & multiplicity \\
\hline \multirow{2}{*}{ even } & \multirow{2}{*}{$\begin{array}{l}\frac{1}{a^{n}} \text { BRiffle } \tau_{a}^{+} \\
\text {or } \frac{1}{a^{n}} \text { BRiffle } \tau_{a}^{-} \\
\text {or } \frac{1}{a^{n}} \text { BRiffle } \tilde{\tau}_{a}^{+} \\
\text {or } \frac{1}{a^{n}} \text { BRiffle } \tilde{\tau}_{a}^{-}\end{array}$} & $a^{k-n}$ & {$\left[x^{k}\right] x(x+2)(x+4) \ldots(x+2 n-2)$} \\
\hline & & 0 & $2^{n} n !-\frac{(2 n) !}{2^{n} n !}$ \\
\hline \multirow{3}{*}{ odd } & $\begin{array}{c}\frac{1}{a^{n}} \text { BRiffle } \tau_{a}^{+} \\
\text {or } \frac{1}{a^{n}} \text { BRiffle } \tilde{\tau}_{a}^{+}\end{array}$ & $a^{k-n}$ & {$\left[x^{k}\right](x+1)(x+3) \ldots(x+2 n-1)$} \\
\hline & \multirow{2}{*}{$\begin{array}{l}\frac{a}{a^{n}} \text { BRiffle } \tau_{a}^{-} \\
\text {or } \frac{1}{a^{n}} \text { BRiffle } \tilde{\tau}_{a}^{-}\end{array}$} & $a^{k-n}$ & {$\left[x^{k}\right](x+n-1)(x+1)(x+3) \ldots(x+2 n-3)^{1}$} \\
\hline & & $-a^{k-n}$ & {$\left[x^{k}\right] n(x+1)(x+3) \ldots(x+2 n-3)$} \\
\hline
\end{tabular}

Table 1: Multiplicities of the eigenvalues of the hyperoctahedral riffle-shuffles

\subsection{Multiplicities of Eigenvalues}

Because $\mathcal{B}_{n}$ is not a basis of $\mathcal{H}_{n}$ (as we're ignoring decks with repeated cards), Proposition 25 does not apply to determine the multiplicities of the hyperoctahedral riffle-shuffles. Instead, we enumerate the signed permutations with the required type of Lyndon factors as listed in Corollary 41.

Theorem 45. Write $[g] f$ to mean the cofficient of the monomial $g$ in the power series $f$. The (algebraic) multiplicities of the eigenvalues of the hyperoctahedral riffle-shuffles are as given in Table 1 .

To prove these multiplicities, let $c(n, k)$ be the signless Stirling number of the first $k i n d$, i.e. the number of permutations of $n$ with $k$ left-to-right minima. Recall that, in a word with distinct letters, the Lyndon factors start precisely at the left-to-right minima, hence $c(n, k)$ is also the number of permutations of $n$ with $k$ Lyndon factors. Important for deducing the generating functions of the multiplicities is the following generating function of $c(n, k)$

$$
\sum_{k} c(n, k) x^{k}=x(x+1) \ldots(x+n-1) .
$$

We define a hyperoctahedral analogue of $c(n, k)$ : let $\mathcal{C}^{+}(n, k, \bar{k})\left(\operatorname{resp} . \mathcal{C}^{-}(n, k, \bar{k})\right)$ be the set of signed permutations of $n$ having $k$ Lyndon factors with an odd number of positive (resp. negative) letters, and $\bar{k}$ Lyndon factors with an even number of positive (resp. negative) letters. Note that changing the sign of all letters gives a bijection between $\mathcal{C}^{+}(n, k, \bar{k})$ and $\mathcal{C}^{-}(n, k, \bar{k})$, so let $C(n, k, \bar{k})$ denote their common size. Observe $C(n, k, \bar{k})=C(n, \bar{k}, k)$, by changing the sign of the first letter in each factor.

Lemma 46. The hyperoctahedral Stirling numbers are related to the type A Stirling numbers by

$$
C(n, k, \bar{k})=2^{n-k-\bar{k}} c(n, k+\bar{k})\left(\begin{array}{c}
k+\bar{k} \\
k
\end{array}\right) .
$$

\footnotetext{
${ }^{1}$ This is a signless version of OEIS sequence A039762
} 
Proof. Given $k$ and $\bar{k}$, fix any sequence of $k$ positive signs and $\bar{k}$ negative signs. There are $\left(\begin{array}{c}k+\bar{k} \\ k\end{array}\right)$ such sequences. We show below that, for any such sequence, there are $2^{n-k-\bar{k}} c(n, k+$ $\vec{k}$ ) signed permutations whose Lyndon factors $u_{i}$ have an odd number (resp. even number) of positive letters if the $i$ th term of the sequence is positive (resp. negative).

First, there are $c(n, k+\bar{k})$ permutations with $k+\bar{k}$ Lyndon factors in total. The factor of $2^{n-k-\bar{k}}$ then enumerates the signs that can be assigned: within each Lyndon factor, all but the last letter can have arbitrary sign; the sign of the last letter is then determined by the requirement that this factor have an odd (or even) number of positive letters.

Lemma 47. The hyperoctahedral Stirling numbers satisfy the recursion

$$
C(n, k, \bar{k})=C(n-1, k-1, \bar{k})+C(n-1, k, \bar{k}-1)+2(n-1) C(n-1, k, \bar{k}) .
$$

Proof. We construct a bijection between the relevant sets. Each element of $\mathcal{C}^{+}(n, k, \bar{k})$ is obtained in precisely one of the following manners:

- Adding $n$ to the start of an element of $\mathcal{C}^{+}(n-1, k-1, \bar{k})-n$ then becomes a new left-to-right minima, i.e. a new Lyndon factor with one positive letter.

- Adding $\bar{n}$ to the start of an element of $\mathcal{C}^{+}(n-1, k, \bar{k}-1)-\bar{n}$ is then a new Lyndon factor with one negative and no positive letters.

- To an element of $\mathcal{C}^{+}(n-1, k, \bar{k})$, add $\bar{n}$ between any two letters, or at the end. This does not produce any new left-to-right minima, so $\bar{n}$ is inserted into one of the existing Lyndon factors, and does not change its parity of its positive letters.

- To an element of $\mathcal{C}^{+}(n-1, k, \bar{k})$, add $n$ between any two letters, or at the end, and also change the sign of the preceding letter, which is necessarily within the same Lyndon factor. This sign change ensures that the parity of positive letters remains unchanged in this factor.

Proof of Theorem 45. Fix a even. From Theorems 29 and 26, and Corollary 41, the multiplicity of the eigenvalue $a^{k-n}$ is $\left|\mathcal{C}^{-}(n, 0, k)\right|$ for $\frac{1}{a^{n}}$ BRiffle $\tau_{a}^{+}$and $\frac{1}{a^{n}}$ BRiffle $\tau_{a}^{-}$, and $\left|\mathcal{C}^{+}(n, k, 0)\right|$ for $\frac{1}{a^{n}}$ BRiffle $\tilde{\tau}_{a}^{+}$and $\frac{1}{a^{n}}$ BRiffle $\tilde{\tau}_{a}^{-}$. By the symmetry remarks before Lemma 46 , their common generating function is

$$
\begin{aligned}
\sum_{k} C(n, k, 0) x^{k} & =\sum_{k} 2^{n-k} c(n, k) x^{k} \\
& =2^{n} \sum_{k} c(n, k)\left(\frac{x}{2}\right)^{k} \\
& =2^{n} \frac{x}{2}\left(\frac{x}{2}+1\right) \ldots\left(\frac{x}{2}+n-1\right) \\
& =x(x+2)(x+4) \ldots(x+2 n-2) .
\end{aligned}
$$

0 is the only other eigenvalue. So that the multiplicities of all eigenvalues sum to $2^{n} n$ !, the multiplicity of 0 must be $2^{n} n !-1 \cdot 3 \cdots(2 n-1)$. 
Now consider $\frac{1}{a^{n}}$ BRiffle $\tilde{\tau}_{a}^{-}$for $a$ odd. From Theorem 27 and Corollary 41, the multiplicities of the eigenvalues $a^{k-n}$ and $-a^{k-n}$, respectively, are $\sum_{\bar{k} \text { even }}\left|\mathcal{C}^{+}(n, k, \bar{k})\right|$ and $\sum_{\bar{k} \text { odd }}\left|\mathcal{C}^{+}(n, k, \bar{k})\right|$. It can be proved by induction on $n$ that

$$
\begin{aligned}
& \sum_{\bar{k} \text { odd }} C(n, k, \bar{k})=n \sum_{\bar{k}} C(n-1, k, \bar{k}), \\
& \sum_{\bar{k} \text { even }} C(n, k, \bar{k})=(n-1) \sum_{\bar{k}} C(n-1, k, \bar{k})+\sum_{\bar{k}} C(n-1, k-1, \bar{k}) ;
\end{aligned}
$$

briefly, use (27) to rewrite the left hand side in terms of $C(n-1,-,-)$, apply the inductive hypothesis to obtain sums of $C(n-2,-,-)$, over all values of $\bar{k}$, then collect terms using (27) to obtain terms of the form $C(n-1,-,-)$. Then the claimed generating functions come from observing that

$$
\begin{aligned}
\sum_{k, \bar{k}} C(n-1, k, \bar{k}) x^{k} & =\sum_{k, \bar{k}} 2^{n-1-k-\bar{k}} c(n-1, k+\bar{k})\left(\begin{array}{c}
k+\bar{k} \\
k
\end{array}\right) x^{k} \\
& =\sum_{j} 2^{n-1-j} c(n-1, j)(x+1)^{j} \\
& =2^{n-1} \sum_{k} c(n-1, j)\left(\frac{x+1}{2}\right)^{j} \\
& =2^{n-1} \frac{x+1}{2}\left(\frac{x+1}{2}+1\right) \ldots\left(\frac{x+1}{2}+n-2\right) \\
& =(x+1)(x+3) \ldots(x+2 n-3) .
\end{aligned}
$$

The multiplicity of $a^{k-n}$ for $\frac{1}{a^{n}}$ BRiffle $\tilde{\tau}_{a}^{+}$is the sum of the multiplicities of $a^{k-n}$ and $-a^{k-n}$ for $\frac{1}{a^{n}}$ BRiffle $\tilde{\tau}_{a}^{-}$. The cases of $\frac{1}{a^{n}}$ BRiffle $\tau_{a}^{-}$and $\frac{1}{a^{n}}$ BRiffle $\tau_{a}^{+}$follow from the symmetry remarks before Lemma 46 .

\subsection{Subdominant Eigenfunctions and Expectations of Descents}

The paper [DPR14, Exs. 5.8-5.9, Prop. 5.10] identified the eigenvectors of type A riffleshuffles with large eigenvalues, and expressed some of them in terms of two classical permutation statistics, namely descents and peaks. This section derives one hyperoctahedral analogue.

First, to clarify what is meant by "large" eigenvalue:

Definition 48. An eigenvalue $\lambda$ of a Markov chain is subdominant if $|\lambda|<1$ and $|\lambda|>\left|\lambda^{\prime}\right|$ for all other eigenvalues $\lambda^{\prime} \neq 1,-1$.

Informally, subdominant eigenvalues have maximal absolute value after 1 . Their values and corresponding eigenspaces have the largest influence on the convergence rate.

The corresponding eigenvectors are expressed in terms of the following terminology: 
Definition 49. If $w=w_{1} \ldots w_{n}$ where $w_{i}$ denotes the $i$ th letter of $w$, then a consecutive subword of $w$ is a word of the form $w_{i} w_{i+1} \ldots w_{j}$ for some $i<j$.

For example, if $w=435 \overline{1} 6 \overline{7} \overline{2}$, then $35 \overline{1}$ and $6 \overline{7} \overline{2}$ are consecutive subwords of $w$, but $36 \overline{7}$ is not.

Definition 50. Given a word $w$, its consecutive subword $i j$ is a descent if $i>j$. This applies whether $i$ and $j$ are positive and negative letters. Let des $(w)$ denote the number of descents in $w$.

For example, the descents of $435 \overline{1} 6 \overline{7} \overline{2}$ are $43,5 \overline{1}$, and $6 \overline{7}$, so $\operatorname{des}(w)=3$. Notice that this differs from the definition of descent in Coxeter type B, where additionally the first letter is a descent if it is negative.

Notation 51. For $i<j$, define

$$
\mathbf{f}_{i j}^{+}(w):= \begin{cases}1 & \text { if any of } i j, \overline{i j} \text { is a consecutive subword in } w \\ -1 & \text { if any of } j i, \bar{j} \bar{i} \text { is a consecutive subword in } w \\ 0 & \text { otherwise }\end{cases}
$$

and

$$
\mathbf{f}_{i j}^{-}(w):= \begin{cases}1 & \text { if any of } \overline{i j}, i \bar{j} \text { is a consecutive subword in } w \\ -1 & \text { if any of } j \bar{i}, \bar{j} i \text { is a consecutive subword in } w \\ 0 & \text { otherwise }\end{cases}
$$

And for $i \neq j$, define

$$
\tilde{\mathbf{f}}_{i j}(w):= \begin{cases}1 & \text { if any of } i j, \overline{i j}, \overline{j i}, \bar{j} \bar{i} \text { is a consecutive subword in } w \\ -1 & \text { if any of } j i, j \bar{i}, i \bar{j}, \overline{i j} \text { is a consecutive subword in } w \\ 0 & \text { otherwise. }\end{cases}
$$

And for all $i$, define

$$
\mathbf{g}_{i}(w):= \begin{cases}1 & \text { if } i \text { is the first or last letter of } w \\ -1 & \text { if } \bar{i} \text { is the first or last letter of } w \\ 0 & \text { otherwise. }\end{cases}
$$

Proposition 52. The subdominant eigenvalues of the hyperoctahedral riffle-shuffles, and a basis of associated eigenfunctions, are as given in Table 2.

The proof is at the end of this section.

Proposition 53. Fix a positive integer $a$, and let $\left\{X_{t}\right\}$ denote a-handed riffle-shuffle with flip, i.e. the Markov chain associated to $\frac{1}{a^{n}}$ BRiffle $\tilde{\tau}_{a}^{+}$or $\frac{1}{a^{n}}$ BRiffle $\tilde{\tau}_{a}^{-}$on $\mathcal{B}_{n}$ according to Section 5.1. Then the "normalised number of descents" functions $w \mapsto \operatorname{des}(w)-\frac{n-1}{2}$ is an eigenfunction of $\left\{X_{t}\right\}$ of eigenvalue $1 /$ a. Hence

$$
\operatorname{Expect}\left(\operatorname{des} X_{t} \mid X_{0}=w_{0}\right)=\left(1-a^{-t}\right) \frac{n-1}{2}+a^{-t} \operatorname{des}\left(w_{0}\right) \text {. }
$$




\begin{tabular}{|c|c|c|c|}
\hline parity of $a$ & shuffle & $\begin{array}{l}\text { subdominant } \\
\text { eigenvalues }\end{array}$ & basis of eigenfunctions \\
\hline even & $\frac{1}{a^{n}}$ BRiffle $\tau_{a}^{+}$or $\frac{1}{a^{n}}$ BRiffle $\tau_{a}^{-}$ & $1 / a$ & $\left\{\mathbf{f}_{i j}^{+}\right\} \cup\left\{\mathbf{f}_{i j}^{-}\right\}$ \\
\hline \multirow{3}{*}{ odd } & $\frac{1}{a^{n}}$ BRiffle $\tau_{a}^{+}$ & $1 / a$ & $\left\{\mathbf{f}_{i j}^{+}\right\} \cup\left\{\mathbf{f}_{i j}^{-}\right\} \cup\left\{\mathbf{g}_{i}\right\}$ \\
\hline & \multirow{2}{*}{$\frac{1}{a^{n}}$ BRiffle $\tau_{a}^{-}$} & $1 / a$ & $\left\{\mathbf{f}_{i j}^{+}\right\} \cup\left\{\mathbf{f}_{i j}^{-}\right\}$ \\
\hline & & $-1 / a$ & $\left\{\mathbf{g}_{i}\right\}$ \\
\hline even & $\frac{1}{a^{n}}$ BRiffle $\tilde{\tau}_{a}^{+}$or $\frac{1}{a^{n}}$ BRiffle $\tilde{\tau}_{a}^{-}$ & $1 / a$ & $\left\{\tilde{\mathbf{f}}_{i j}\right\}$ \\
\hline \multirow{3}{*}{ odd } & $\frac{1}{a^{n}}$ BRiffle $\tilde{\tau}_{a}^{+}$ & $1 / a$ & $\left\{\tilde{\mathbf{f}}_{i j}\right\} \cup\left\{\mathbf{g}_{i}\right\}$ \\
\hline & \multirow{2}{*}{$\frac{1}{a^{n}}$ BRiffle $\tilde{\tau}_{a}^{-}$} & $1 / a$ & $\left\{\tilde{\mathbf{f}}_{i j}\right\}$ \\
\hline & & $-1 / a$ & $\left\{\mathbf{g}_{i}\right\}$ \\
\hline
\end{tabular}

Table 2: Subdominant eigenvalues for hyperoctahedral riffle-shuffles, and bases for their eigenfunctions

Proof. We show that

$$
\operatorname{des}(w)-\frac{n-1}{2}=\sum_{i<j} \tilde{\mathbf{f}}_{i j}(w)
$$

for all $w \in \mathcal{B}_{n}$. Note that the subwords which cause $\tilde{\mathbf{f}}_{i j}(w)=-1$, namely $j i, j \bar{i}, i \bar{j}$ and $\overline{i j}$, are all descents if $i<j$. And every descent of $w$ is of one of these 4 types, for some $i<j$. Opposingly, all consecutive subwords of two letters that are not descents (i.e. "ascents") contribute 1 to $\tilde{\mathbf{f}}_{i j}(w)$ for some $i<j$. Hence $\sum_{i<j} \tilde{\mathbf{f}}_{i j}(w)$ is the difference between the number of ascents and the number of descents in $w$. Since each pair of consecutive letters in $w$ is either an ascent or a descent, the number of ascents and descents together total $n-1$. Hence this difference is $(n-1-\operatorname{des}(w))-\operatorname{des}(w)$.

As for the expectation assertion: by the linearity of expectations, then by Proposition 37 ,

$$
\begin{aligned}
\operatorname{Expect}\left(\operatorname{des} X_{t} \mid X_{0}=w_{0}\right) & =\operatorname{Expect}\left(\sum_{i<j} \tilde{\mathbf{f}}_{i j}\left(X_{t}\right) \mid X_{0}=w\right)+\operatorname{Expect}\left(\frac{n-1}{2} \mid X_{0}=w\right) \\
& =a^{-t} \sum_{i<j} \tilde{\mathbf{f}}_{i j}\left(w_{0}\right)+\frac{n-1}{2} .
\end{aligned}
$$

Proof of Proposition 52. According to Theorems 26, 27 and 29, for the four operators $\frac{1}{a^{n}}$ BRiffle $\tau_{a}^{+}, \frac{1}{a^{n}}$ BRiffle $\tau_{a}^{-}, \frac{1}{a^{n}}$ BRiffle $\tilde{\tau}_{a}^{+}$and $\frac{1}{a^{n}}$ BRiffle $\tilde{\tau}_{a}^{-}$:

- When $a$ is even, the only subdominant eigenvalue is $1 / a$, and it corresponds to $k=n-1$ and $\bar{k}=0$, i.e. the corresponding eigenvectors are formed from $n-1$ $\tau$-invariant (or $\tilde{\tau}$-invariant) primitives, and no $\tau$-negating (or $\tilde{\tau}$-negating) primitives. 
- When $a$ is odd, the subdominant eigenvalues all correspond to $k=n-1$ and $\bar{k}$ can take any value, i.e. the corresponding eigenvectors are formed from $n-1 \tau$ invariant (or $\tilde{\tau}$-invariant) primitives, and any number of $\tau$-negating (or $\tilde{\tau}$-negating) primitives. However, since $k+\bar{k} \leqslant n$ (as each primitive has degree at least one, and the degree of their product is $n$ ), the only possible values of $\bar{k}$ are 0 and 1 . When $\bar{k}=0$, the eigenvalue is $1 / a$ for all four operator families. When $\bar{k}=1$, the eigenvalue is $1 / a$ for $\frac{1}{a^{n}}$ BRiffle $\tau_{a}^{+}$and $\frac{1}{a^{n}}$ BRiffle $\tilde{\tau}_{a}^{+}$, and is $-1 / a$ for $\frac{1}{a^{n}}$ BRiffle $\tau_{a}^{-}$, and $\frac{1}{a^{n}}$ BRiffle $\tilde{\tau}_{a}^{-}$.

The proof will be completed by showing the following:

i) $\left\{\tilde{\mathbf{f}}_{i j} \mid i<j\right\} \cup\left\{-\tilde{\mathbf{f}}_{i j} \mid j<i\right\}$ are precisely all the eigenvectors, for BRiffle $\tilde{\tau}_{a}^{+}$and BRiffle $\tilde{\tau}_{a}^{-}$, given by Algorithm 42 when $k=n-1$ and $\bar{k}=0$.

ii) $\left\{\mathbf{f}_{i j}^{+} \mid i<j\right\}$ (resp. $\left.\left\{\mathbf{f}_{i j}^{-} \mid i<j\right\}\right)$ are, up to scaling, the sums (resp. differences) of pairs of eigenvectors, for BRiffle $\tau_{a}^{+}$and BRiffle $\tau_{a}^{-}$, given by Algorithm 42 when $k=n-1$ and $\bar{k}=0$.

iii) $\left\{\mathbf{g}_{i}\right\}$ are precisely all the eigenvectors, for BRiffle $\tilde{\tau}_{a}^{+}$, BRiffle $\tilde{\tau}_{a}^{-}$, BRiffle $\tau_{a}^{+}$and BRiffle $\tau_{a}^{-}$when $a$ is odd, given by Algorithm 42 when $k=n-1$ and $\bar{k}=1$.

And here are the proofs of these three assertions.

i) $k=n-1$ and $\bar{k}=0$ means that the indexing word has $n-1$ Lyndon factors, whose standard-bracketings are all $\tilde{\tau}$-invariant. So, by the third line of Corollary 41, each Lyndon factor consists of an odd number of positive letters. Necessarily, $n-2$ of these factors must be single positive letters, and the remaining factor consists of one positive and one negative letter - i.e. it is $i \bar{j}$ or $\bar{i} j$, where $i<j$. Take the first case; the standard-bracketing of $i \bar{j}$ is

$$
[i+\bar{i}, j-\bar{j}]=i j-i \bar{j}+\bar{i} j-\bar{i} \bar{j}-j i-j \bar{i}+\bar{j} i+\overline{j i} .
$$

Observe that, if $w$ consists precisely of $i$ or $\bar{i}$ once and $j$ or $\bar{j}$ once, then $\tilde{\mathbf{f}}_{i j}(w)$ is precisely the coefficient of $w$ in (32).

Since all primitives involved are $\tilde{\tau}$-invariant, the formulas in parts a, b, c of Theorem 26 all agree: the common eigenvector for BRiffle $\tilde{\tau}_{a}^{+}$and BRiffle $\tilde{\tau}_{a}^{-}$is the symmetrised product of (32) with $r+\bar{r}$, ranging over all $r \neq i, j$. In this product, the terms in (32) stay as consecutive subwords, and the letters distinct from $i, j$ may have any order, with equal coefficient. Thus, the coefficient of a word in this eigenvector is given by $\tilde{\mathbf{f}}_{i j}$.

As for $\overline{i j}$, its standard-bracketing is $[i-\bar{i}, j+\bar{j}]=-[j+\bar{j}, i-\bar{i}]$, which differs from (32) only by a global sign and the exchange of $i$ and $j$. So, by the same argument as above, $-\tilde{\mathbf{f}}_{j i}(w)$ describes the coefficient of $w$ in this eigenvector. 
ii) Similar to case i) above, these eigenvectors are produced from $n-1$ Lyndon factors whose standard-bracketing are all $\tau$-invariant. By the first line of Corollary 41, each Lyndon factor consists of an even number of negative letters. As in case i), $n-2$ of the factors are single positive letters, and the remaining factor is ij or $\overline{i j}$. Note that

$$
\begin{aligned}
& \operatorname{stdbrac}(i j)=i j+\bar{i} j+i \bar{j}+\overline{i j}-j i-j \bar{i}-\bar{j} i-\overline{j i} \\
& \operatorname{stdbrac}(\overline{i j})=i j-\bar{i} j-i \bar{j}+\overline{i j}-j i+j \bar{i}+\bar{j} i-\overline{j i} .
\end{aligned}
$$

So $p^{+}:=\frac{1}{2}(\operatorname{stdbrac}(i j)+\operatorname{stdbrac}(\overline{i j}))$ and $p^{-}:=\frac{1}{2}(\operatorname{stdbrac}(i j)-\operatorname{stdbrac}(\overline{i j}))$ have coefficients as decribed by $\mathbf{f}_{i j}^{+}$and $\mathbf{f}_{i j}^{-}$, respectively, when $w$ consists only of $i$ or $\bar{i}$ once and $j$ or $\bar{j}$ once. The extension to words longer than $w$ is as in case i): take the symmetrised product of $p^{+}$or $p^{-}$with all $r+\bar{r}$ for $r \neq i, j$, following Theorems 27 and 29. Note that (with the obvious notation)

$$
\begin{aligned}
\operatorname{sym}\left\{p^{+}\right\} \cup\{r+\bar{r} \mid r \neq i, j\}=\operatorname{sym}\{\operatorname{stdbrac}(i j)\} \cup\{r+\bar{r} \mid r \neq i, j\} \\
+\operatorname{sym}\{\operatorname{stdbrac}(\overline{i j})\} \cup\{r+\bar{r} \mid r \neq i, j\}
\end{aligned}
$$

and similarly for $p^{-}$, because taking symmetrised product is linear in each argument.

iii) $k=n-1$ and $\bar{k}=1$ means that the indexing word has $n$ Lyndon factors, so each factor must be a single letter. From Corollary 41, the standard-bracketing of a single positive letter is both $\tau$-invariant and $\tilde{\tau}$-invariant, and the standard-bracketing of a single negative letter is both $\tau$-negating and $\tilde{\tau}$-negating. Hence, these $n$ Lyndon factors are precisely one negative letter, say $\bar{i}$, and $n-1$ positive letters. Since $\bar{k}=1$, the formula in Theorem 26.i.c (for BRiffle $\tilde{\tau}_{a}^{-}$) and (12) (for BRiffle $\tau_{a}^{+}$and BRiffle $\left.\tau_{a}^{-}\right)$both simplify to $(i-\bar{i}) s+s(i-\bar{i})$, where $s$ denotes the symmetrised product of $r+\bar{r}$ over all $r \neq i$. The coefficient of a word in this eigenvector is given by $\mathbf{g}_{i}$.

\subsection{An Eigenfunction related to Signs}

In [FKLP21, Th. 1.4, 5.8], the authors give the probability, under type A riffle-shuffles or under BRiffle $\tilde{\tau}_{a}^{+}$for $a$ odd, that the resulting deck has sign 1, when viewed as a permutation or signed permutation. It is noted there that the type A case may be proved using a related eigenfunction, constructed using Hopf-algebraic methods. Jimmy He informed us that the hyperoctahedral result might also be obtainable in this manner, which we do below, extending the result of [FKLP21] to BRiffle $\tilde{\tau}_{a}^{-}$and to riffle-shuffles with rotation when the number of cards is even.

Definition 54. Given a signed permutation $w=w_{1} \ldots w_{n}$, its $\operatorname{sign} \operatorname{sgn}(w)$ is the product of the sign of the permutation $\left|w_{1}\right| \ldots\left|w_{n}\right| \in \mathfrak{S}_{n}$ and the parity of signed integers in $w$.

For example, $\operatorname{sgn}(\overline{4} 35 \overline{1} \overline{2})=1$ because the permutation 43512 has sign -1 , and there are an odd number of signed integers in $\overline{4} 35 \overline{1} \overline{2}$. 


\begin{tabular}{|c|c|c|}
\hline parity of $a$ & shuffle & eigenvalue $\beta$ \\
\hline \hline odd or even & $\frac{1}{a^{n}}$ BRiffle $\tau_{a}^{+}$or $\frac{1}{a^{n}}$ BRiffle $\tau_{a}^{-}$ & $a^{-\frac{n}{2}}$ for $n$ even \\
\hline even & $\frac{1}{a^{n}}$ BRiffle $\tilde{\tau}_{a}^{+}$or $\frac{1}{a^{n}}$ BRiffle $\tilde{\tau}_{a}^{-}$ & 0 \\
\hline \multirow{2}{*}{ odd } & $\frac{1}{a^{n}}$ BRiffle $\tilde{\tau}_{a}^{+}$ & $a^{-n}$ \\
\cline { 2 - 4 } & $\frac{1}{a^{n}}$ BRiffle $\tilde{\tau}_{a}^{-}$ & $(-1)^{\left\lceil\frac{n}{2}\right\rceil} a^{-n}$ \\
\hline
\end{tabular}

Table 3: Eigenvalues corresponding to the sign function

Proposition 55. sgn is an eigenfunction of the a-handed riffle-shuffles with flip or with rotation, for eigenvalues $\beta$ as listed in Table 3. Hence, if $\left\{X_{t}\right\}$ denotes one of the shuffles above,

$$
\operatorname{Prob}\left(\operatorname{sgn} X_{t}=1 \mid \operatorname{sgn} X_{0}=1\right)=\frac{1}{2}\left(1+\beta^{t}\right) .
$$

Proof. Given elements $p_{1}, \ldots, p_{k}$ in the signed free associative algebra, let $\operatorname{sym}\left\{p_{1}, \ldots, p_{k}\right\}$ denote the symmetrised product $\sum_{\sigma \in \mathfrak{S}_{k}} p_{\sigma(1)} \ldots p_{\sigma(k)}$. We also write $\sigma_{i}$ instead of $\sigma(i)$. Let $\mathfrak{A}_{n}$ denote the alternating group on $n$ objects, i.e. the set of even permutations. Below, we first show that

$$
\begin{gathered}
\sum_{\sigma \in \mathfrak{A}_{n}} \operatorname{sym}\left\{\left[\sigma_{1}-\overline{\sigma_{1}}, \sigma_{2}-\overline{\sigma_{2}}\right],\left[\sigma_{3}-\overline{\sigma_{3}}, \sigma_{4}-\overline{\sigma_{4}}\right], \ldots\right. \\
\left.\ldots,\left[\sigma_{n-1}-\overline{\sigma_{n-1}}, \sigma_{n}-\overline{\sigma_{n}}\right]\right\} \text { if } n \text { is even; } \\
\sum_{\sigma \in \mathfrak{A}_{n}} \operatorname{sym}\left\{\left[\sigma_{1}-\overline{\sigma_{1}}, \sigma_{2}-\overline{\sigma_{2}}\right],\left[\sigma_{3}-\overline{\sigma_{3}}, \sigma_{4}-\overline{\sigma_{4}}\right], \ldots\right. \\
\left.\ldots,\left[\sigma_{n-2}-\overline{\sigma_{n-2}}, \sigma_{n-1}-\overline{\sigma_{n-1}}\right], \sigma_{n}-\overline{\sigma_{n}},\right\} \text { if } n \text { is odd }
\end{gathered}
$$

is a multiple of the sign function, then we verify its eigenvalue.

First observe that, for even $n, \operatorname{sym}\{12,34, \ldots, n-1 n\}$ is a sum over permutations that can be obtained from the identity by some number of transpositions of $i i+1$ with $j j+1$ (for odd $i, j$ ), and each such transposition-pair is an even permutation, so all permutations appearing in $\operatorname{sym}\{12,34, \ldots, n-1 n\}$ are even. Next, $\operatorname{sym}\{[1,2],[3,4], \ldots,[n-1, n]\}$ additionally allows transposing $i$ and $i+1$ (for odd $i$ ), but this incurs a minus sign. Finally,

$$
\operatorname{sym}\{[1-\overline{1}, 2-\overline{2}],[3-\overline{3}, 4-\overline{4}], \ldots,[(n-1)-(\overline{n-1}), n-\bar{n}]\}
$$

additionally allows conversion of $i$ to $\bar{i}$, again at the cost of a minus sign. Hence, for every signed permutation that appears in (33), its coefficient is equal to its sign. We then symmetrise (33) over all even permutations, to ensure that all signed permutations appear in the result, with coefficients proportional to their sign.

For the case for odd $n$ : each permutation appearing in $\operatorname{sym}\{12,34, \ldots, n-2 n-1, n\}$ is a product of some transposition-pairs $i i+1$ with $j j+1$ and one cycle of the form 
$(i i+1 \ldots n)$ for odd $i$. This cycle has sign 1 , so all such permutation products have sign 1 . The remainder of the argument applies unchanged.

To deduce the eigenvalues: $\sigma_{i}-\bar{\sigma}_{i}$ is $\tau$-negating and $\tilde{\tau}$-negating, so by Proposition 40 $\left[\sigma_{i}-\bar{\sigma}_{i}, \sigma_{j}-\bar{\sigma}_{j}\right]$ is $\tau$-invariant and $\tilde{\tau}$-negating. By Lemma 30, the symmetrised product of $\frac{n}{2} \tau$-invariant primitives is an eigenvector of $\frac{1}{a^{n}}$ BRiffle $\tau_{a}^{+}$or $\frac{1}{a^{n}}$ BRiffle $\tau_{a}^{-}$, with eigenvalue $a^{-n} a^{\frac{n}{2}}$. And by Theorem 26, the product of $\left\lceil\frac{n}{2}\right\rceil \tilde{\tau}$-negating primitives is an eigenvector of $\frac{1}{a^{n}}$ BRiffle $\tilde{\tau}_{a}^{+}$and $\frac{1}{a^{n}}$ BRiffle $\tilde{\tau}_{a}^{-}$, with eigenvalues as in Table 3 .

For the probability assertion:

$$
\begin{aligned}
\operatorname{Expect}\left(\operatorname{sgn} X_{t}\right) & =\operatorname{Prob}\left(\operatorname{sgn} X_{t}=1\right)-\operatorname{Prob}\left(\operatorname{sgn} X_{t}=-1\right) \\
& =2 \operatorname{Prob}\left(\operatorname{sgn} X_{t}=1\right)-1
\end{aligned}
$$

and by the linearity of expectations as in Proposition 53, Expect $\left(\operatorname{sgn} X_{t}\right)=\beta^{t} \operatorname{sgn} X_{0}$.

\section{Acknowledgements}

We thank Ronan Conlon and Eric Marberg for inspiring this project, and Marcelo Aguiar, Persi Diaconis, Jason Fulman, Rafael González D’León, Jimmy He, Nadia Lafrenière and the referee for many helpful comments. SAGE computer software [ $\left.\mathrm{S}^{+} 19\right]$ was very useful, especially the combinatorial Hopf algebras coded by Aaron Lauve and Franco Saliola.

\section{References}

[AA17] M. Aguiar and F. Ardila. Hopf monoids and generalized permutahedra. arXiv:1709.07504, September 2017.

[ABS06] M. Aguiar, N. Bergeron, and F. Sottile. Combinatorial Hopf algebras and generalized Dehn-Sommerville relations. Compos. Math., 142(1):1-30, 2006.

[AL15] M. Aguiar and A. Lauve. The characteristic polynomial of the Adams operators on graded connected Hopf algebras. Algebra Number Theory, 9(3):547-583, 2015.

[AS05] M. Aguiar and F. Sottile. Cocommutative Hopf algebras of permutations and trees. J. Algebraic Combin., 22(4):451-470, 2005.

[BB92] F. Bergeron and N. Bergeron. Orthogonal idempotents in the descent algebra of $B_{n}$ and applications. J. Pure Appl. Algebra, 79(2):109-129, 1992.

[BD92] D. Bayer and P. Diaconis. Trailing the dovetail shuffle to its lair. Ann. Appl. Probab., 2(2):294-313, 1992.

[BHR99] P. Bidigare, P. Hanlon, and D. Rockmore. A combinatorial description of the spectrum for the Tsetlin library and its generalization to hyperplane arrangements. Duke Math. J., 99(1):135-174, 1999.

[BW17] J. R. Britnell and M. Wildon. Bell numbers, partition moves and the eigenvalues of the random-to-top shuffle in Dynkin types A, B and D. J. Combin. Theory Ser. A, 148:116-144, 2017. 
[Car07] P. Cartier. A primer of Hopf algebras. In Frontiers in Number Theory, Physics, and Geometry. II, pages 537-615. Springer, Berlin, 2007.

[CP17] G. Chatel and V. Pilaud. Cambrian Hopf algebras. Adv. Math., 311:598-633, 2017.

[DFH13] P. Diaconis, J. Fulman, and S. Holmes. Analysis of casino shelf shuffling machines. Ann. Appl. Probab., 23(4):1692-1720, 2013.

[DPR14] P. Diaconis, C. Y. A. Pang, and A. Ram. Hopf algebras and Markov chains: two examples and a theory. J. Algebraic Combin., 39(3):527-585, 2014.

[DS] P. Diaconis and B. Steinberg. Coloured shuffles and random walks on semigroups. Unpublished Manuscript.

[DS18] A. B. Dieker and F. V. Saliola. Spectral analysis of random-to-random Markov chains. Adv. Math., 323:427-485, 2018.

[Dur08] R. Durrett. Genome rearrangement. In Probability Models for DNA Sequence Evolution, pages 355-397. Springer, New York, 2008.

[Fil96] J. A. Fill. An exact formula for the move-to-front rule for self-organizing lists. J. Theoret. Probab., 9(1):113-160, 1996.

[FKLP21] J. Fulman, G. B. Kim, S. Lee, and T. K. Petersen. On the joint distribution of descents and signs of permutations. Electron. J. Combin., 28(3):\#P3.37, 2021.

[Ful01] J. Fulman. Applications of the Brauer complex: card shuffling, permutation statistics, and dynamical systems. J. Algebra, 243(1):96-122, 2001.

[Ful02] J. Fulman. Applications of symmetric functions to cycle and increasing subsequence structure after shuffles. J. Algebraic Combin., 16(2):165-194, 2002.

[GR14] D. Grinberg and V. Reiner. Hopf algebras in combinatorics. arXiv: 1409.8356, September 2014.

[Hiv07] F. Hivert. An introduction to combinatorial Hopf algebras - examples and realizations. In Physics and theoretical computer science, volume 7 of NATO Secur. Sci. Ser. D Inf. Commun. Secur., pages 253-274. IOS, Amsterdam, 2007.

[JR79] S. A. Joni and G.-C. Rota. Coalgebras and bialgebras in combinatorics. Stud. Appl. Math., 61(2):93-139, 1979.

[Kna02] A. W. Knapp. Lie groups beyond an introduction, volume 140 of Progress in Mathematics. Birkhäuser Boston Inc., Boston, MA, second edition, 2002.

[Laf20] N. Lafrenière. Eigenvalues of symmetrized shuffling operators. Sém. Lothar. Combin., 82B:Art. 78, 12, 2020.

[Lod98] J.-L. Loday. Cyclic homology, volume 301 of Grundlehren der Mathematischen Wissenschaften [Fundamental Principles of Mathematical Sciences]. SpringerVerlag, Berlin, second edition, 1998. Appendix E by María O. Ronco, Chapter 13 by the author in collaboration with Teimuraz Pirashvili. 
[Lot97] M. Lothaire. Combinatorics on Words. Cambridge Mathematical Library. Cambridge University Press, Cambridge, 1997. With a foreword by Roger Lyndon and a preface by Dominique Perrin; corrected reprint of the 1983 original, with a new preface by Perrin.

[LPW09] D. A. Levin, Y. Peres, and E. L. Wilmer. Markov chains and mixing times. American Mathematical Society, Providence, RI, 2009. With a chapter by James G. Propp and David B. Wilson.

[LR98] J.-L. Loday and M. O. Ronco. Hopf algebra of the planar binary trees. Adv. Math., 139(2):293-309, 1998.

[LSK02] B. Larget, D. L. Simon, and J. B. Kadane. Bayesian phylogenetic inference from animal mitochondrial genome arrangements. Journal of the Royal Statistical Society: Series B, 64(4):681-693, 2002.

[Moy15] M. Moynihan. The colored Eulerian descent algebra. J. Algebraic Combin., 42(3):671-694, 2015.

[MR95a] C. Malvenuto and C. Reutenauer. Duality between quasi-symmetric functions and the Solomon descent algebra. J. Algebra, 177(3):967-982, 1995.

[MR95b] R. Mantaci and C. Reutenauer. A generalization of Solomon's algebra for hyperoctahedral groups and other wreath products. Comm. Algebra, 23(1):2756, 1995.

[Pan18] C. Y. A. Pang. Markov chains from descent operators on combinatorial Hopf algebras. arXiv:1609.04312, August 2018.

[Pat94] F. Patras. L'algèbre des descentes d'une bigèbre graduée. J. Algebra, 170(2):547-566, 1994.

[PP98] R. M. Phatarfod and A. J. Pryde. On some multi-request move-to-front heuristics. J. Appl. Probab., 35(4):911-918, 1998.

[PP99] A. J. Pryde and R. M. Phatarfod. Multiplicities of eigenvalues of some linear search schemes. Linear Algebra Appl., 291(1-3):115-124, 1999.

$\left[\mathrm{S}^{+} 19\right] \quad$ W. Stein et al. Sage Mathematics Software (Version 8.7). The Sage Development Team, 2019. http://www.sagemath.org.

[Sch93] W. R. Schmitt. Hopf algebras of combinatorial structures. Canad. J. Math., 45(2):412-428, 1993.

[Sol76] L. Solomon. A Mackey formula in the group ring of a Coxeter group. J. Algebra, 41(2):255-264, 1976.

[Swe69] M. E. Sweedler. Hopf algebras. Mathematics Lecture Note Series. W. A. Benjamin, Inc., New York, 1969.

[Wil04] D. B. Wilson. Mixing times of Lozenge tiling and card shuffling Markov chains. Ann. Appl. Probab., 14(1):274-325, 2004. 